Photonic-Doppler-Velocimetry, Paraxial-Scalar Diffraction Theory and Simulation

W. P. Ambrose

September 10, 2015 
This document was prepared as an account of work sponsored by an agency of the United States government. Neither the United States government nor Lawrence Livermore National Security, LLC, nor any of their employees makes any warranty, expressed or implied, or assumes any legal liability or responsibility for the accuracy, completeness, or usefulness of any information, apparatus, product, or process disclosed, or represents that its use would not infringe privately owned rights. Reference herein to any specific commercial product, process, or service by trade name, trademark, manufacturer, or otherwise does not necessarily constitute or imply its endorsement, recommendation, or favoring by the United States government or Lawrence Livermore National Security, LLC. The views and opinions of authors expressed herein do not necessarily state or reflect those of the United States government or Lawrence Livermore National Security, LLC, and shall not be used for advertising or product endorsement purposes.

This work performed under the auspices of the U.S. Department of Energy by Lawrence Livermore National Laboratory under Contract DE-AC52-07NA27344. 


\title{
Photonic-Doppler-Velocimetry, \\ Paraxial-Scalar Diffraction Theory and Simulation
}

\author{
W. P. Ambrose \\ Lawrence Livermore National Laboratory
}

September 10, 2015

@ 2015 , W. P. Ambrose @ Lawrence Livermore National Laboratory. 


\section{CONTENTS}

PREFACE

Summary description of the contents of the report

Glossary

Coordinate and sign conventions

Key results

Index of key results with section numbers

Acknowledgements

CHAPTER 1. Introduction

1.1 What is Photonic Doppler Velocimetry? What does it measure? and the goals for this work

1.2 Qualitative description of spectral analysis in PDV, and the concept of instantaneous peak frequency

1.3 Example of PDV experimental results with frequency deviations at amplitude valleys

1.4 Working hypothesis and plan for the derivation of instantaneous peak frequency for PDV

1.5 A sideband spectral peak is connected with time varying difference in phases of scattered light and reference field

1.6 A brief detour: The Doppler shift in one dimension has two different contributions from space-like and time-like terms

CHAPTER 2. The instantaneous peak frequency

2.1 Relating the instantaneous signal frequency to the scattered light field - time domain picture

2.2 Relating instantaneous-sideband peak frequency in a heterodyne-detection signalspectrum to time dependent optical fields - frequency domain picture

2.3 The instantaneous peak frequency is equivalent to the real part of a sum involving a delicate balance of complex-valued weights

CHAPTER 3. The detected field

3.1 Classical electromagnetic wave propagation and point-wise matter-field scattering 
3.2 Setting up a field calculation for a flat surface traveling at constant uniform velocity with confocal illumination and observation using a probe with lens and optical fiber

3.3 Diffraction of the aperture field at the fiber

3.3.1 Review of scalar diffraction theory

3.3.2 Including the fiber lens in the aperture-fiber diffraction calculation

3.3.3 Comment on not integrating over the fiber end face

3.4 Scalar light-field components scattered from target to aperture in the paraxial approximation

3.5 Closed-form solutions for propagation time $T_{i a}$ and its derivative $\dot{T}_{i a}$ including target-lens delay $\delta\left(\vec{r}_{a}\right) / c$

3.6 The complete superposition of fields scattered from target points $\vec{r}_{i}$ that arrive simultaneously at aperture points $\vec{r}_{a}$

3.7 Reversing the order of integration: a geometric description of the superposition of scattered fields from one target point $\vec{r}_{i 0}$

CHAPTER 4. Expressing $\omega_{p}$ in terms of the diffraction result for $E_{r}{ }^{*} E_{s}$

4.1 Instantaneous PDV-signal frequency expressed in terms of the time dependent scattered fields

4.2 What happens to the expression for $\omega_{p}$ for small angles?

4.3 Values of $\omega_{i a}$ for scattering points moving in a Gaussian laser field

and scattering light into lens aperture points

4.4 What happens to the value of $\omega_{p}$ at large angles?

CHAPTER 5. Simulation results

5.1 Simulation parameters that were fixed for all simulations

5.2 Tilted surface with no roughness

5.3 Tilted surface with increasing amounts of roughness

6. BIBLIOGRAPHY 


\section{Preface (abstract)}

In this report I describe current progress on a paraxial, scalar-field theory suitable for simulating what is measured in Photonic Doppler Velocimetry (PDV) experiments in three dimensions. I have introduced a number of approximations in this work in order to bring the total computation time for one experiment down to around 20 hours. My goals were: to develop an approximate method of calculating the peak frequency in a spectral sideband at an instant of time based on an optical diffraction theory for a moving target, to compare the 'measured' velocity to the 'input' velocity to gain insights into how and to what precision PDV measures the component of the mass velocity along the optical axis, and to investigate the effects of small amounts of roughness on the measured velocity. This report illustrates the progress I have made in describing how to perform such calculations with a full three dimensional picture including tilted target, tilted mass velocity (not necessarily in the same direction), and small amounts of surface roughness. With the method established for a calculation at one instant of time, measured velocities can be simulated for a sequence of times, similar to the process of sampling velocities in experiments. Improvements in these methods are certainly possible at hugely increased computational cost. I am hopeful that readers appreciate the insights possible at the current level of approximation.

The first chapter is mainly qualitative background for folks who may not have participated in the analysis of PDV data. I also use these methods as inspiration for a proof of a concept in Chapter 2. In the first chapter I illustrate qualitatively how experimentalists think about the relationship between the velocity and signal frequency in a heterodyne velocimetry (PDV) experiment. Chapter 1 includes a detailed look at the usual one dimensional heuristic arguments often used to motivate a relationship between "the signal frequency" and "the target velocity." Chapter 1 includes also an example of experimental data to motivate a question examined in Chapter 5 "how does target surface roughness affect the measurement?" Folks familiar with PDV and the usual methods of analysis who want to bypass Chapter 1 may find it useful to examine section 1.6, as I use the one dimensional picture for comparison with three dimensions later in Chapter 4.

In Chapter 2, I define an instantaneous-peak frequency $\omega_{p}$ in the signal-sideband spectrum, and its relation to a signal cross term between the scattered light field $E_{s}$ and reference field $E_{r}^{*}$ in the detected power, i.e., $E_{r}{ }^{*} E_{s}$. In section 2.1, I motivate a possible expression for $\omega_{p}$ without proof (so the reader can see where we are headed), and then prove the idea rigorously in section 2.2. Section 2.1 is a hand-wavey argument starting from an assumption that it is true that $\omega_{p}=\partial \Phi(t) / \partial t$, where $\Phi(t)$ is the difference in phase between $E_{r}^{*}$ and $E_{s}$, from which we find easily that $\omega_{p}=\operatorname{Im}\left[\left(\partial\left(E_{r}^{*} E_{s}\right) / \partial t\right) /\left(E_{r}^{*} E_{s}\right)\right]$. It is much easier to see how this expression arises in the time domain. For those uncomfortable starting with an assumption that the spectral-peak frequency is the derivative of the phase difference, the proof is offered in sections 2.2. Section 2.2 can be viewed as the rigorous proof of the relationship between $\omega_{p}$ and $E_{r}^{*} E_{s}$, or alternatively proof that $\omega_{p}=\partial \Phi(t) / \partial t$. The proof allows readers to see underlying conditions that need to be true to arrive at either of these 
equivalent expressions. We introduce another assumption in Chapter 2 that is proved in chapter 4 , that underlying the sideband signal peaks is a narrow spectrum of frequencies arising from different scattered light paths. Chapter 2 ends with an illustration of how the different frequencies in a narrow spectrum of frequencies are combined with complex-valued weights to arrive at "the frequency" $\omega_{p}$ at the peak of the signal spectrum. From these results, we gain insights into what is actually measured in a heterodyne measurement: the peak in a sideband spectrum results from a sum of spectral contributions weighted by complex values related to the laser field.

Having connected $\omega_{p}$ with $E_{r}^{*} E_{s}$ in Chapter 2, in Chapter 3 I develop the relationship of the scattered optical field at the detector to the motion of the target in the laser field. For the scattered optical fields, I developed a diffraction theory for a moving target and a PDV probe with lens and optical fiber. I solved for an analytical expression for the optical delay $T_{i a}$ between a scattering event and a lens aperture point for a special case: a flat plate traveling with uniform constant velocity in any direction and orientation (with or without surface roughness); optical probe with single mode optical fiber and thin lenses; and any laser- field function in three dimensions.

In Chapter 4, I combine two relationships developed in Chapters 2 and 3, i.e., $\omega_{p}$ related to $E_{r}^{*} E_{s}$, and $E_{r}^{*} E_{s}$ related to the motion of the target in the laser field, to connect $\omega_{p}$ to the motion of the target. We examine the relative importance of the terms in the product $E_{r}^{*} E_{s}$ and write down a good approximation for $\omega_{p}$. We examine three cases: We examine the form of $E_{r}{ }^{*} E_{s}$ in the limit of a perfectly smooth and symmetric experiment (similar to an idealized one dimensional case). Contributions from motion transverse to the laser field disappear. We note that this limit has similarities to the expansion in harmonic fields at the end of Chapter 2 and hypothesize that the underlying spectrum is narrow but not harmonic. In order to explore the behavior in a finite beam in the small angle limit, we introduce a Gaussian laser field with wave-front curvature and find that the plane wave terms in the laser-field phase dominate the result connecting a component of the velocity along the optical axis to the measured frequency (section 4.3). Using a finite width beam, we do find a small amount of spectral width (on the order of $1 \%$ ) mainly due to integration of optical delays over the lens aperture. Up to this point in the discussion, we have ignored another term in the integral for the peak frequency related to variation in the laser field amplitude seen by the moving target which becomes important for large angles between the particle velocity and the optical axis (more than 45 degrees). We note at the end of chapter 4 that the complexities due to transverse motion through the beam needs further study to understand it's effect on the measured velocity at large angles. We will not explore further what happens at large angles in this report.

For the simulations in Chapter 5, I used a Gaussian-beam field-function for the laser beam confocal with the probe. PDV typically is used in a confocal paraxial optical geometry with unknown (uncontrolled) optical polarization at the target and fields that are nearly transverse to the optical axis. I used a scalar field theory that is good to approximately $0.5 \%$ in the amplitudes, and exact in the field phases. 
(Readers may play with the obliquity factor if they want to improve on the amplitude approximation, but they should also examine carefully the approximations introduced in Chapter 3 ). We illustrate an example using simulations of a slightly asymmetric picture with tilted probe, tilted particle velocity and roughness added to the target surface. We observe that the delicate cancellations in the underlying spectrum may be disrupted by roughness leading to momentary deviations in the measured velocity of up to a few percent.

These calculations were performed within certain limits: We work in the high-power classical limit. We detect light with no sense of the discreteness of photons and no shot noise. Fields propagate as classical electromagnetic waves. We include reflection of light using point-wise light-matter interactions (with or without roughness). We borrow concepts consistent with relativistic electrodynamics to write down the scattered light field. The optical system uses several "thin" and "paraxial" approximations. And we work in the "low" velocity limit compared to the speed of light.

Table 1 is a glossary with an index to section numbers, with additional notes on conventions, assumptions, and key results.

\begin{tabular}{|c|c|}
\hline$E_{s}(t)$ & Total scattered light field at the detector. Sections: preface, 1.5, 2.1, 2.2, \\
\hline$E_{r}(t)$ & Harmonic reference field at the detector. Sections: preface, $1.5,2.1,2.2$ \\
\hline $\begin{array}{l}\text { Harmonic and non } \\
\text { harmonic fields }\end{array}$ & Sections: preface, $1.1,1.2,1.5,2.3,3.1,3.3 .1,3.3 .2,4.1,4.2,4.4$ \\
\hline $\begin{array}{l}E_{\ell}(\vec{r}) \operatorname{Exp}\left(i \phi_{\ell}(\vec{r}, t)\right) \\
=E_{g}(\vec{r}) \operatorname{Exp}\left(i \phi_{g}(\vec{r}, t)\right)\end{array}$ & $\begin{array}{l}\text { The laser field expressed as a Gaussian beam. This is a harmonic function in the } \\
\text { laboratory frame, but has a narrow spectrum (not harmonic) in the frame of the } \\
\text { target due to motion of the laser. } \\
\text { Sections preface, } 1.1,1.2,1.5,3.2,3.4,3.5,3.6,4.1,4.3,5.0\end{array}$ \\
\hline$\lambda$ & Laser wavelength. Section 1.2 \\
\hline$g\left(t ; t_{0}, \tau\right)$ & Analysis window function. Section 1.2. \\
\hline$\tau$ & Window width. Section 1.2, 1.4, 2.2 \\
\hline $\begin{array}{l}\text { Total detected power for } \\
\text { a transverse scalar field }\end{array}$ & $p(t)=\left|E_{s}+E_{r}\right|^{2}=\left(E_{r}+E_{s}\right) \cdot\left(E_{r}+E_{s}\right) *$ Sections 1.5 \\
\hline $\begin{array}{l}\text { Total detected power in } \\
\text { frequency domain }\end{array}$ & $P(\omega)=P_{r}+P_{s}+P_{+}+P_{-}$. Sections 1.2, 2.2 \\
\hline Signal power & $p_{+} \sim E_{r}^{*} \cdot E_{s}=\left|E_{r}\right||A(t)| e^{i \Phi(t)}$ Sections 1.5 \\
\hline$\Phi(t)$ & Phase difference between scattered light and reference. Sections 1.5, 2.2 \\
\hline$+(k x-\omega t), \omega=k c$ & Sign convention for advancing phase and frequency transforms. Section 1.6, 2.2 \\
\hline $\begin{array}{l}\text { Peak in the signal } \\
\text { spectrum }\end{array}$ & $\begin{array}{l}\omega_{p}:\left.\frac{\partial P_{+}(\omega)}{\partial t}\right|_{\omega_{p}}=0, \text { where } P_{+}(\omega)=\frac{1}{\sqrt{2 \pi}} \int_{-\infty}^{\infty} d t e^{\omega t} g\left(t ; t_{o}, \tau\right) p_{+}(t) \\
\text { Sections: preface, } 1.2,1.4,2.1\end{array}$ \\
\hline$\omega_{m} \approx \omega_{p}$ & $\begin{array}{l}\text { Average of the spectral sideband is approximately the peak position, which } \\
\text { becomes an identity in the instantaneous limit. Section } 1.2,1.4,2.2\end{array}$ \\
\hline
\end{tabular}




\begin{tabular}{|c|c|}
\hline $\begin{array}{l}\text { Instantaneous peak } \\
\text { frequency in the signal } \\
\text { spectrum }\end{array}$ & $\begin{array}{l}\tau \rightarrow 0, \quad \omega_{p}(t)=-\frac{\partial}{\partial t} \Phi=\operatorname{Re}\left[\left.\frac{1}{\left(E_{r}^{*} E_{s}\right)} i \frac{\partial}{\partial t}\left(E_{r}^{*} E_{s}\right)\right|_{t=t_{0}}\right] \\
\text { Sections: preface, } 2.1 \text { (easy version with assumptions), } 2.2 \text { (proved) }\end{array}$ \\
\hline$\hat{x}$ & $\begin{array}{l}\text { Optical axis for confocal optical system consisting of single-mode fiber, lens } \\
\text { aperture, target lens, and fiber lens. Sections: } 3.2\end{array}$ \\
\hline$\vec{u}_{p}$ & $\begin{array}{l}\text { Constant uniform target particle velocity for each and all particles. Sections: } 3.2 \text {, } \\
3.6\end{array}$ \\
\hline$\vec{\beta}$ & Dimensionless $\vec{u}_{p}$ relative to the speed of light $c$, i.e., $\vec{\beta}=\vec{u}_{p} / c$ Sections: 3.2 \\
\hline$\beta_{x}$ & $\begin{array}{l}\beta_{x}=\vec{\beta} \cdot \hat{x} \text { component of } \vec{\beta} \text { along the optical axis (confocal with laser field axis). } \\
\text { Sections: } 1.6,3.2,4.3 \text { and } 5.1\end{array}$ \\
\hline$\vec{r}_{i 0}$ & $\begin{array}{l}\text { Initial positions of target particles } i \text { from which light will be scattered (later). } \\
\text { Sections: } 3.2\end{array}$ \\
\hline$\vec{r}_{a}$ & $\begin{array}{l}\text { Lens aperture point (between infinitesimally close target lens and fiber lens). } \\
\text { Sections: } 3.2,3.6\end{array}$ \\
\hline$\hat{n}$ & Target surface normal direction. Sections: $3.2,3.5,3.6$ \\
\hline$x_{i 0}=x_{i 0}\left(y_{i 0}, z_{i 0}\right)$ & Initial position surface. Section $3.5,5.0$ \\
\hline$\delta x_{i 0}=\delta x_{i 0}\left(y_{i 0}, z_{i 0}\right)$ & Target surface roughness added to surface $x_{i 0} \rightarrow x_{i 0}+\delta x_{i 0}$. Chapter 5 . \\
\hline$x_{00}$ & Initial position on the beam center line. Section 3.5 \\
\hline$k \delta_{f}$ and $k \delta$ & Fiber lens and target lens phase delays. Section 3.2, 3.2.2 \\
\hline$c T_{i a}=\left|\vec{r}_{a}-\vec{r}_{i}\right|+\delta\left(\vec{r}_{a}\right)$ & $\begin{array}{l}\text { Optical delay between scattered light point } i \text { and lens-aperture point } a \text { through } \\
\text { target-lens delay } \delta\left(\vec{r}_{a}\right) \text {. Infinitesimally close and thin lens approximation. } \\
\text { Sections: preface, } 1.6,2.2,2.3,3.3,3.4,3.5,3.6\end{array}$ \\
\hline$\vec{r}_{i}=\vec{r}_{i 0}+\left(t_{a}-T_{i a}\right) \vec{u}_{p}$ & $\begin{array}{l}\text { Position where light arriving at } \vec{r}_{a} \text { had been scattered at earlier time }\left(t_{a}-T_{i a}\right) \text {. } \\
\text { Sections: } 3.2,3.6\end{array}$ \\
\hline$E_{\ell}\left(\vec{r}_{i}\right) e^{i \phi_{\ell}\left(\vec{r}_{i}, t_{a}-T_{i a}\right)}$ & $\begin{array}{l}\text { Laser field at point-like space-time scattering event at earlier time }\left(t_{a}-T_{i a}\right) \text { and } \\
\text { position } \vec{r}_{i}=\vec{r}_{i 0}+\left(t_{a}-T_{i a}\right) \vec{u}_{p} \text {. Sections } 3.3,3.4,4.1,4.2\end{array}$ \\
\hline$R_{o}$ & $\begin{array}{l}\text { Distance along optical axis from fiber to aperture. Fiber lens focal distance. } \\
\text { Sections 3.2, 3.3.2 }\end{array}$ \\
\hline$R_{P_{o} A}$ & $\begin{array}{l}\text { Distance from points in the aperture to the end face of the single mode optical } \\
\text { fiber. Sections } 3.2,3.3 .2\end{array}$ \\
\hline$R_{f} / C$ & $\begin{array}{l}\text { Constant time delay value from all points in aperture to fiber through fiber lens. } \\
\text { Sections } 3.3,3.3 .2,3.6\end{array}$ \\
\hline $\begin{array}{l}\text { Superposition of light } \\
\text { scattered from laser field } \\
\text { summed over target } \\
\text { points } \vec{r}_{i 0} \text { and aperture } \\
\text { points } \vec{r}_{a} \text {. }\end{array}$ & $\begin{array}{l}E_{s}=\frac{-1}{2 \pi} R_{o} \iint_{A} d y_{a} d z_{a} \frac{1}{R_{P_{o} A}^{2}}\left\{\frac{1}{R_{P_{o} A}}+2 \frac{1}{c} \frac{\partial}{\partial t}\right\} \iint d y_{i 0} d z_{i 0} \frac{1}{n_{x}} \frac{O_{i a}}{R_{i a}} E_{\ell}\left(\vec{r}_{i}\right) e^{i \phi_{\ell}\left(\vec{r}_{i}, t_{a}-T_{i a}\right)} \\
\text { This is a diffraction result derived for a moving target. Optical delays from aperture } \\
\text { to detector are arranged all to be the same using a fiber lens. The relevant delay } \\
\text { becomes the delay from scattering event to aperture point through target lens. } \\
\text { Sections: } 3.3 .1,3.3 .2,3.5,3.6\end{array}$ \\
\hline Obliquity factor & $O_{i a}$. Introduced in section: $3.5 . O_{i a}=1$ ignored in section 5. \\
\hline
\end{tabular}




\begin{tabular}{|c|c|}
\hline $\begin{array}{l}\text { Solving for the delay from } \\
\text { scattering points to } \\
\text { aperture points though } \\
\text { lens }\end{array}$ & $\begin{array}{l}\text { Two coupled relations: } \vec{r}_{i}=\vec{r}_{i 0}+\left(t_{a}-T_{i a}\right) \vec{u}_{p}, \quad c T_{i a}=\left|\vec{r}_{a}-\vec{r}_{i}\right|+\delta\left(\vec{r}_{a}\right) \\
\Rightarrow T_{i a}=T_{i a}\left(\vec{u}_{p}, \vec{r}_{i 0}, \vec{r}_{a}, t_{a}\right) \\
\text { Sections 3.4, 3.6 }\end{array}$ \\
\hline$\frac{\partial T_{i a}}{\partial t}=\vec{\beta} \cdot \frac{\vec{r}_{i}-\vec{r}_{a}}{\left|\vec{r}_{i}-\vec{r}_{a}\right|}$ & $\begin{array}{l}\text { The rate of change of optical delay in low velocity limit } u_{p}<<c \text { is component of } \\
\text { the dimensionless velocity along light-scattering path from } \vec{r}_{i} \text { to } \vec{r}_{a} \text {. } \\
\text { Sections: } 3.4,4.2\end{array}$ \\
\hline $\begin{array}{l}\text { Good approximation to } \\
\text { instantaneous peak } \\
\text { frequency. Exact in } \\
\text { phase. Terms with } \\
\text { amplitude } 10^{-4} \text { smaller } \\
\text { were ignored. Error from } \\
\text { choice of } O_{i a} \sim 0.5 \% \text { in } \\
\text { amplitudes. }\end{array}$ & 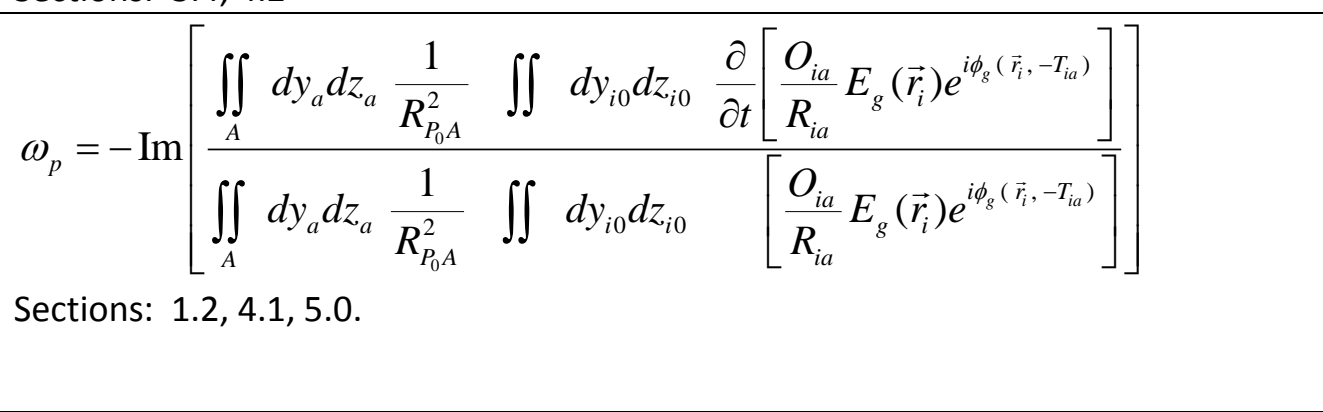 \\
\hline$\omega_{i a}=-\partial \varphi_{g}\left(\vec{r}_{i},-T_{i a}\right) / \partial t$ & $\begin{array}{l}\text { Rate of change of the difference in phase between the reference field and a } \\
\text { scattered light field that propagates from } \vec{r}_{i} \text { to } \vec{r}_{a} \text {. Variations up to a few percent } \\
\text { are connected with the optical delay } T_{i a} \text { dependence on aperture positions } \vec{r}_{a} \text {. } \\
\text { Sections: } 4.1,4.2,4.3,5.0\end{array}$ \\
\hline $\begin{array}{l}\text { Signal frequency is closely } \\
\text { related to the component } \\
\text { of the velocity along the } \\
\text { optical axis. }\end{array}$ & $\begin{array}{l}\omega_{p} \cong 2 \omega_{r} \beta_{x} \text {. } \\
\text { For nearly symmetric situations, there are two dominant terms from the derivative } \\
\text { in the numerator. One is related to the space-like nearly-plane wave like term in } \\
\text { the laser beam phase, } \frac{\partial}{\partial t} \vec{k} \cdot \vec{r}_{i} \approx \omega_{r} \hat{k} \cdot \vec{\beta} \text {. The other is from the time rate of change } \\
\text { in the optical delay } \omega_{r} \frac{\partial T_{i a}}{\partial t}=\omega_{r} \vec{\beta} \cdot \frac{\left(\vec{r}_{i}-\vec{r}_{a}\right)}{\left|\vec{r}_{i}-\vec{r}_{a}\right|} \approx \omega_{r} \beta_{x} \text {. } \\
\text { Sections: } 1.6,3.1,4.3\end{array}$ \\
\hline $\begin{array}{l}\text { integrand densities for } \\
\omega_{p} \text { at } y_{i o}, z_{i o} \text { obtained } \\
\text { after integrating over the } \\
\text { aperture }\end{array}$ & $\begin{array}{l}\left(d \omega_{y_{i 0}, z_{i 0}} / d A\right) \Delta y_{i 0} \Delta z_{i 0} \\
=-\operatorname{Im}\left[\frac{\sum_{y i, z j} \frac{1}{R_{P_{0} A}^{2}} \frac{c}{\partial}}{\sum_{y i, z j} \sum_{y a, z a} \frac{1}{R_{P_{0} A}^{2}}}\right.\end{array}$ \\
\hline
\end{tabular}

\section{Acknowledgements}

It has been approximately a year since I became interested in this problem: ideas about what is measured in PDV are based largely on heuristic one dimensional notions, PDV measures a three dimensional situation, and there are occasionally "odd" things in PDV data for which there is no accepted understanding. In particular, there is a growing, quiet realization among folks who analyze 
PDV data that there are occasionally "weird" unexplained and momentary deviations of the velocity away from the normal trend on the order of a few percent during momentary "dark" periods that seem too systematic to be from random noise contributions. I claim that these effects can be seen in collections of presentations of results from different researchers shared at PDV workshops. The latest workshop was held at the University of Las Vegas, Nevada, in June 2014. Momentary deviations in the measured velocity are not understood and often just ignored (the argument is that it is "unphysical" for a massive target's velocity to suddenly speed up and slow down again in a mere nanosecond. And why does this happen as the signal takes a dive?). An example of these effects is illustrated in the first chapter using PDV data obtained in a gas-gun shot at Lawrence Livermore National Laboratory. I would like to thank the following artists and scientists for careful preparation and execution of that shot: Rocky Beckleman, Justin Jones, Bob Nafzinger, Paul Benevento, Phil Watts, Gary Roads, Gerard Jacobson, Sam Weaver, Steve Caldwell, and Ricky Chau. The author acknowledges additional discussions and inspiration for this work in conversations with Reed Patterson, Neil Holmes, Jeff Nguyen, Ricky Chau, and Minta Akin. A significant turning point for this work was probably born in a conversation with Reed and Neil who asked "...but what about the lens?" The diffraction theory in Chapter 3 is an answer. While working with Neil and David Holtkamp on gas gun experiments that included PDV at Los Alamos National Laboratory, I believe I introduced my progress on this topic and that I was on the verge of completing a rigorous derivation for the general result for the instantaneoussideband spectrum peak-frequency (this is now finished in Chapter 2). The author would like to acknowledge Lawrence Livermore National Laboratory for providing the technical resources for this work: the entirety of this work was prepared on a laboratory-supplied laptop computer, much of it performed at night or on weekends. I wrote this report because I believe I have developed a few correct and key mathematical results that I have synthesized into a correct picture for this problem, which may also be useful. I am indebted to Albert Lee and Dennis McNabb for a review of this work to ensure it meets the standards of our institution for dissemination of information in a public forum. This work was self-motivated and was not in response to any specific programmatic need; I blame no one but myself for inaccuracies and errors in the current level of approximation. 


\section{CHAPTER 1. Introduction}

The success and popularity of Photonic Doppler Velocimetry (PDV) are due in part to it's highly reliable "hands off" operational simplicity in moments just before an experiment. Notions about how PDV measures velocity were originally introduced and described by Strand et. al. based on a heuristic, perfectly symmetric, one-dimensional Ersatz. Results for experiments that are not perfectly symmetric have led scientists including Briggs and Dolan to consider such questions as "what does PDV really measure?" [Dolan 2009, Briggs 2009] Dolan also has explored the problem that heterodyne detection introduces surprising mathematical difficulties in areas such as propagation of random signal errors (noise) into the final value for "a velocity," and illustrated the complexities using a Monte Carlo simulation [Dolan 2010]. The answer to a related question "how good is a measurement?" is frequently not addressed using an analytical approach. The answer can be determined experimentally by comparison of deviations between a large number of identical channels or experiments, and is often found to be better than $1 \%$ (for carefully prepared and completely symmetric situations results can be as good as $0.1 \%)$. In this report, we make progress on an answer to the question "what does PDV measure" using a paraxial scalar diffraction theory in an attempt to gain additional insights into the possible origins of errors in measurement.

\subsection{What is Photonic Doppler Velocimetry, What does it measure, and the goals for this work}

An observer shines light on a moving target, the target reflects the light, and returns the light to the sender. The frequency of light scattered from a moving object is Doppler shifted. Photonic Doppler Velocimetry (PDV) is an experimental technique that provides information on the motion of a target via changes in the frequency of scattered light. The Figure 1.1.1 is a cartoon depiction of a PDV probe and target. The probe consisting of single-mode optical fiber and lens mounted in an opaque tube.

Laser light is propagated along a single-mode optical fiber to the probe, the light diverges from the fiber, and is converted to a converging-and-diverging "laser beam" by a lens. Light scattered from a moving target is collected by the same lens. This arrangement with illumination and collection by the same lens is described by the word confocal. A portion of the scattered light is counter-propagated back to a detector on the same optical fiber (via an optical circulator, not shown). The commercially availability of compact optical circulators based on single-mode fiber optic technologies has made it practical to perform optical velocimetry in this confocal geometry [Strand 2006] with a large number of identical channels in a compact space (In a retrospective presentation in June of 2014, Strand claims he was searching for a way to make the concept of PDV a reality when Tony Whitworth introduced him to optical circulators). 


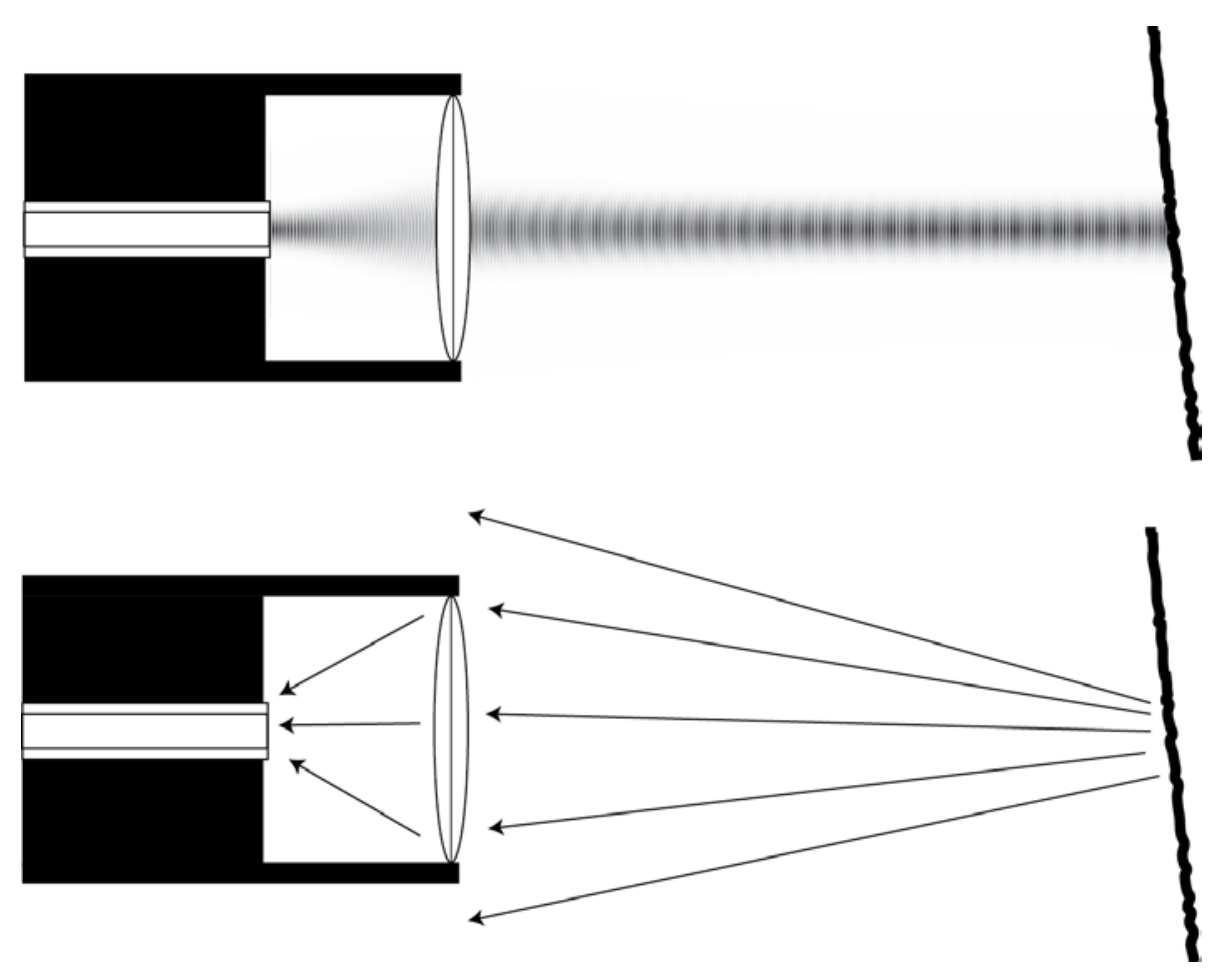

Figure 1.1.1 PDV-probe cartoon with exaggerated features. Upper panel: Laser light emerges from an optical fiber and is focused into a converging and diverging "laser beam." Lower panel: The laser light is scattered from a target (right line). The scattered light fills the lens aperture and some of the laser light is coupled back into the fiber. The reader should note that the use of "straight lines" in (b) is not meant literally, but is related to a way of thinking about integration over paths between surface points and lens-aperture points (see Chapter 3 ).

PDV is based on heterodyne detection [Kingston chapter 3; Yariv sections 11.4 and 11.5]. Scattered light is superposed with a continuous-wave single-frequency laser. The power in the two interfering waves is detected and converted to an electronic signal. An intermediate-frequency signal is generated, oscillating at the difference in frequencies between the scattered light spectrum and the harmonic reference. A primary goal of PDV analysis is based on extracting this intermediate frequency spectrum and relating it to the velocity (or velocities).

PDV is capable of observing targets that are tilted and have velocity that is not parallel to the optic axis. A small amount of roughness can be added to the target surface to spread the scattered light into a larger solid angle, and to maintain the scattered light signal under tilt in confocal optics. A question arises: what does PDV measure when the experiment does not have a perfectly-symmetrical onedimensional configuration? A few experimental tests have been performed with a mixture of tilts and different velocity directions relative to the optic axis of a confocal probe [Dolan 2009, Briggs 2009]. Clearly the positive results of Dolan or Briggs are consistent with this idea: PDV measures the component of the particle velocity along the optic axis with very little dependence on surface tilt. The precision of this conclusion and its general applicability has not been established.

Since the target velocity can change in time (in particular when shock waves are involved), experimentalists often desire "instantaneous" values from PDV data. In a real experiment, the data are 
sampled at discrete times, and there is noise in the detected signal due to the discreteness of electrons and photons (electronic thermal and photon shot noise). Extracting a frequency in PDV involves effectively statistical averaging over short time intervals. Attempting to extract an instantaneous frequency is impractical for real data since it is "sampled" at discrete times. Statistical analysis methods suffer from increased uncertainty as the averaging-time interval is decreased to the sample interval. An esthetic compromise is usually reached with tradeoffs between uncertainties in frequency and time (a tradeoff inherent in a frequency transform when noise is present on the signal).

We will show in this chapter that despite the real limitations in the physical world, the concept of an instantaneous peak frequency is a well-defined mathematical concept and causes no statistical problems for a theory (simulation) of a continuous, noiseless experiment. We believe the instantaneous peak frequency is also unique (we obtain the same result from two different derivations) and can be viewed as the limit of an average frequency in zero-width time interval. In subsequent sections we illustrate the method of simulating PDV with examples. The simulations use three-dimensional classical field theory and diffraction theory for a moving target. In later sections we show how to perform simulations in order to gain insights into how and what PDV measures.

We will show that a three-dimensional theory in the paraxial approximation that employs superposition of point-wise scattering events is consistent with the conclusion that the observed velocity is nearly equal to the component of the velocity along the optic axis. The methods used in this report involve a scalar field theory approximation for a paraxial optical system (propagation nearly along the optical axis). A semi quantitative insight from this work is that conclusions drawn about PDV are based on a delicate balancing of cancellations in a narrow spectrum of fields. Additional work (possibly involving a vector theory) would be required to determine exactly the precision of these conclusions. Errors in this balance become apparent in real experiments when one compares identical (redundant) channels in a nominally symmetric experiment.

In the next few sections, we develop the concept and expressions for an instantaneous peak frequency. This instantaneous peak frequency contains information on the motion of the target in a heterodyning experiment. As a vehicle for introducing frequency transforms and a spectral region of interest, we review qualitatively a method of spectral analysis of PDV data. At first we note in passing that the sideband spectral peaks contain information on the scattered-light phase shifts, i.e., the interesting part of the signal lays in sideband spectral peaks. Next, we introduce electromagnetic fields to the picture and show how sideband spectral peaks arise from a product of two different fields when detecting the optical power (in a subsequent chapter we will show how to calculate these fields). We take a small detour into a one dimensional description of PDV to illustrate how the scattered light phase shifts result predominantly from two different terms with comparable value: from a changing phase in a space-like phase term due to motion of the target in a laser beam (or motion of the laser relative to the target!), and from a changing phase (delay) to the detector from a time-like term in the phase. In a one dimensional highly symmetric confocal picture, these two Doppler shift terms are precisely identical. We take a description of the sideband spectral peak written in terms time varying optical fields into the instantaneous limit, and prove an identity between the instantaneous peak frequency and the time dependent fields. We examine the form of the instantaneous peak frequency using an expansion in 
harmonic functions. We find that the instantaneous sideband peak frequency is similar to a weighted average over complex fields (not probabilities).

\subsection{Qualitative description of spectral analysis in PDV, and \\ the concept of an instantaneous peak frequency}

Our purpose in this section is to motivate a starting point for a derivation for an instantaneous peak frequency. Here we introduce basic ideas often used in the detection and analysis of PDV signals. In PDV, the detected power is from a superposition of two optical fields. The two optical fields are a harmonic reference field, and the scattered field from the target (Doppler shifted). Coherent detection of a reference field and signal field at the same detector is known as heterodyne detection (See for example [Kingston chapter 3, or Yariv sections 11.4 and 11.5]). In PDV, the heterodyne signal is detected in a wide electronic bandwidth (tens of $\mathrm{GHz}$ ), electronically sampled in a sequence of narrow time intervals (sampled in intervals as short as $20 \mathrm{ps}$ ), and a time resolved record is made using analog to digital recording.

As depicted in figure 1.2.1, the detected optical power has a DC offset and oscillatory signal. Riding on the DC offset are interference oscillations between the reference and scattered light fields with an oscillation frequency closely related to the difference between the reference and scattered light frequencies. The higher frequency interference oscillations carry information about the motion of the target, and when the motion changes the frequency is modulated, also. The signal frequency and frequency modulation are of interest. Often the interference oscillations carry unwanted amplitude modulated.

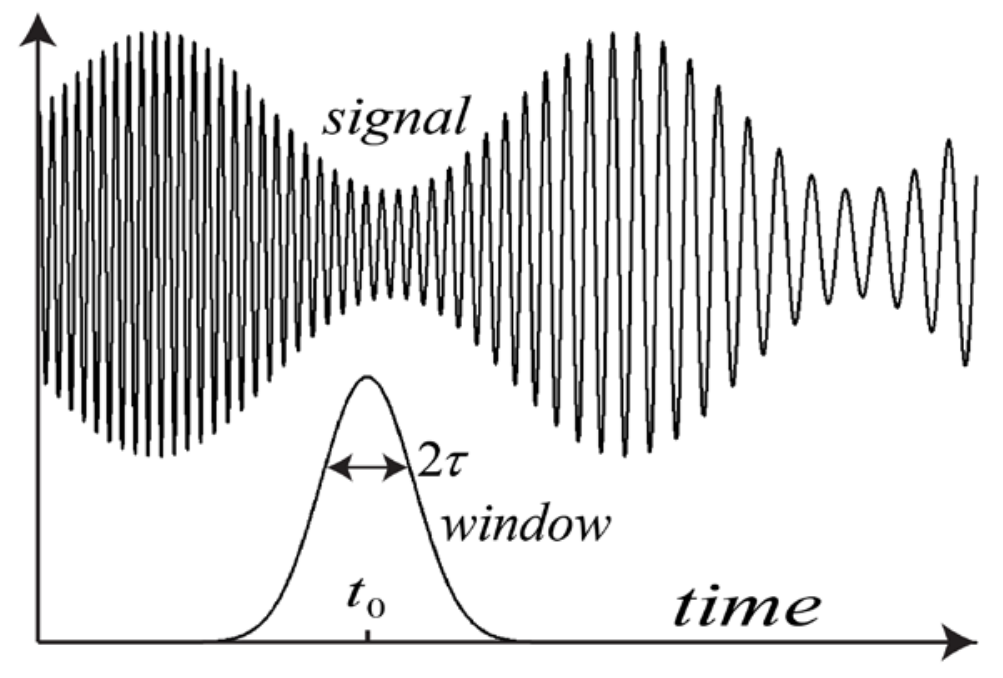

Figure 1.2.1. Cartoon for the detected optical power and a analysis window function. 
PDV often uses a source laser frequency near $193 \mathrm{THz}$ (1.55 micrometers wavelength). For motion at $1 \mathrm{~km} / \mathrm{s}$ in free space, the high speed oscillation is $v \lambda / 2=0.775 \mathrm{GHz}$ (we defer until later detailed incorporation of ideas connecting the high speed oscillation and motion of the target in three dimensions. For this work we avoid the problems of changes of index of refraction in tamping windows - this is a discussion in free space.)

Converting the signal from the time domain to the frequency domain provides a way to distinguish the high-speed signal frequency from amplitude modulation and noise. There is more than one method for extracting spectra. Our claim is that the instantaneous peak frequency derivation for simulations should have a unique value that does not depend on the choice of method of analyzing data. We start with the more "usual" Fourier transform methods to motivate the derivation.

In order to perform the frequency transform on real sampled data, one needs to examine the signal over some interval of time. A simplistic method is to chop out a subdomain of data wide enough to contain at least a few oscillations about a center time $t_{0}$ and associate a frequency transform with $t_{0}$.

Truncation creates "feet" or oscillations in the base of the spectrum. A method used to reduce the feet is to multiply the data by a sliding apodization-window function instead of using truncation. As depicted in Fig. 1.2.1, a window function $g\left(t ; t_{0}, \tau\right)$ with a width parameter $\tau$ is centered at times $t_{0}$. A peaked apodization function $g\left(t ; t_{0}, \tau\right)$ that varies continuously to zero more slowly than discontinuous truncation for $\left|t-t_{0}\right|$ large tends to reduce the "feet". The choice of window is not unique, involves aesthetics, does not change the peak positions, but only changes the shape of the spectral peaks. Since the transform of a Gaussian is a Gaussian function, a Gaussian window creates spectra with no feet. Once a window function is selected, the signal is multiplied by $g\left(t ; t_{0}, \tau\right)$ for a sequence of $t_{0}$ values, transformed, and overlapping spectra are associated with each time $t_{0}$.

The frequency transform is complex-valued (contains phase and amplitude information). For purposes of examining the spectral content, a real-valued spectral power (amplitude squared) may be computed from the complex-valued transform. The DC offset and lower-frequency amplitude modulation and noise will create a delta function and spectral peak straddling zero frequency (labelled $P_{r}$ and $P_{s}(\omega)$ in the Fig. 1.2.2). The desired situation is to have a high-speed signal that is more rapid than the amplitude modulation, in which case signal-sideband spectral peaks $\left(P_{ \pm}(\omega)\right)$ are found positioned symmetrically at positive and negative frequencies and well separated from the baseband spectrum. The peak positions in $P_{ \pm}(\omega)$ move away from zero frequency in proportion to the velocity of the target. To avoid artifacts from nearby spectra, numerical analysis is performed in a region of interest around a peak $P_{+}$ (the box $B$ ). 


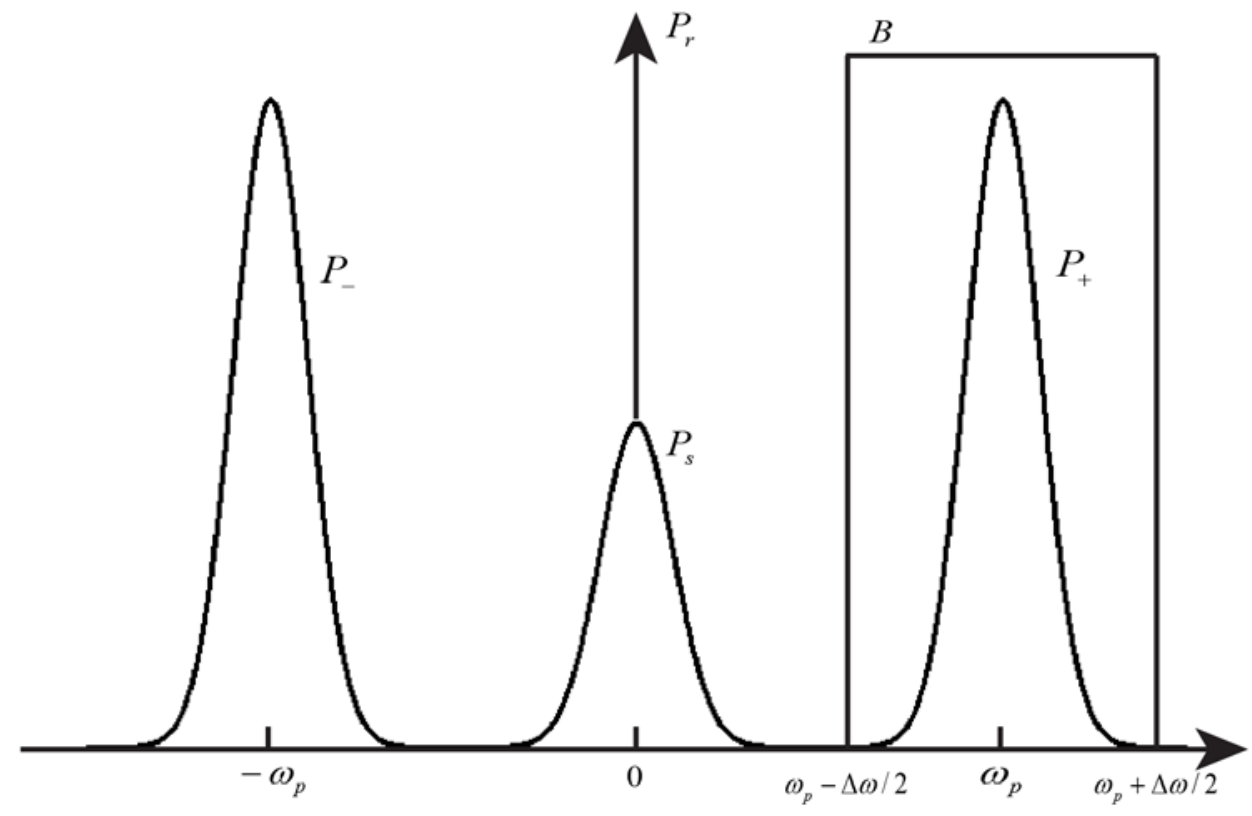

Figure 1.2.2. Cartoon showing the spectra associated with different terms in the detected optical power.

Mainly because of spectral noise (not shown), analysis methods tend to involve statistical measures of the signal-sideband spectrum. Various methods are available for determining an estimate for the signal sideband frequency. A common method is to use non-linear least squares minimization of the differences between a peaked function $G(\omega)$ and the spectral peak $P_{+}(\omega)$ within $B$. From the minimization of a second moment of the differences, the best-fit parameters provide an estimate for the location of the peak frequency $\omega_{p}$. Analysis is made more difficult by the amplitude modulation spectrum $P_{s}(\omega)$ bleeding into the region of interest $B$, by a noisy background, and noise spectrum that may vary with frequency, all of which may be accounted for using a fitting function with more terms and parameters (the cost may be a larger error in $\omega_{p}$ ).

Due to the presence of unwanted signals within the region of interested, an obvious but less readily applicable statistical method is to calculate the mean frequency of the spectrum in the region of interest, $\omega_{m}=\langle\omega\rangle=\int_{B} d \omega \omega P_{+} / \int_{B} d \omega P_{+}$. Using a mean frequency requires a clean separation of the signal sideband peak from other spectra at the edges of $B$, and a good estimate of the noise spectrum. For an instantaneous peak frequency that varies slowly, these methods are expected to have similar values, $\omega_{m} \approx \omega_{p}$. (We will have an occasion to revisit $\omega_{m}$ to support a theory of instantaneous peak frequency). 
We feel it is important to emphasize a subtlety in our terminology to avoid confusion later. The sideband spectrum is obviously a peaked function with a width determined (partly) by the window function. We will show below that there is always an underlying (narrow) spectrum of Doppler shifts in a three dimensional experiment. In the analysis of a PDV experiment, one pulls out one number - the peak position in the sideband spectrum. In the following it is important to note that although we will speak of the underlying spectrum of Doppler shifts, the end goal is to calculate the peak position for this spectrum in order to simulate the process that experimentalists use when attempting to determine "the velocity."

\subsection{Example of PDV experimental results with frequency deviations at amplitude valleys}

An example of PDV data is shown in Figure 1.3.1. Six nominally identical mass-velocity results were obtained at the interface between a flat platinum surface and a lithium fluoride tamping window. A shock wave breaks out of the platinum into the LiF with a mean mass velocity of $1.94 \mathrm{~km} / \mathrm{sec}$. Late-time side shocks and releases arrive after 200 ns. During the planar shock $(<200 \mathrm{~ns})$, there are two types of deviations from the mean. There are random deviations wandering less than 1 percent about the mean. And there are occasional non-random brief spikes larger than 1 percent.

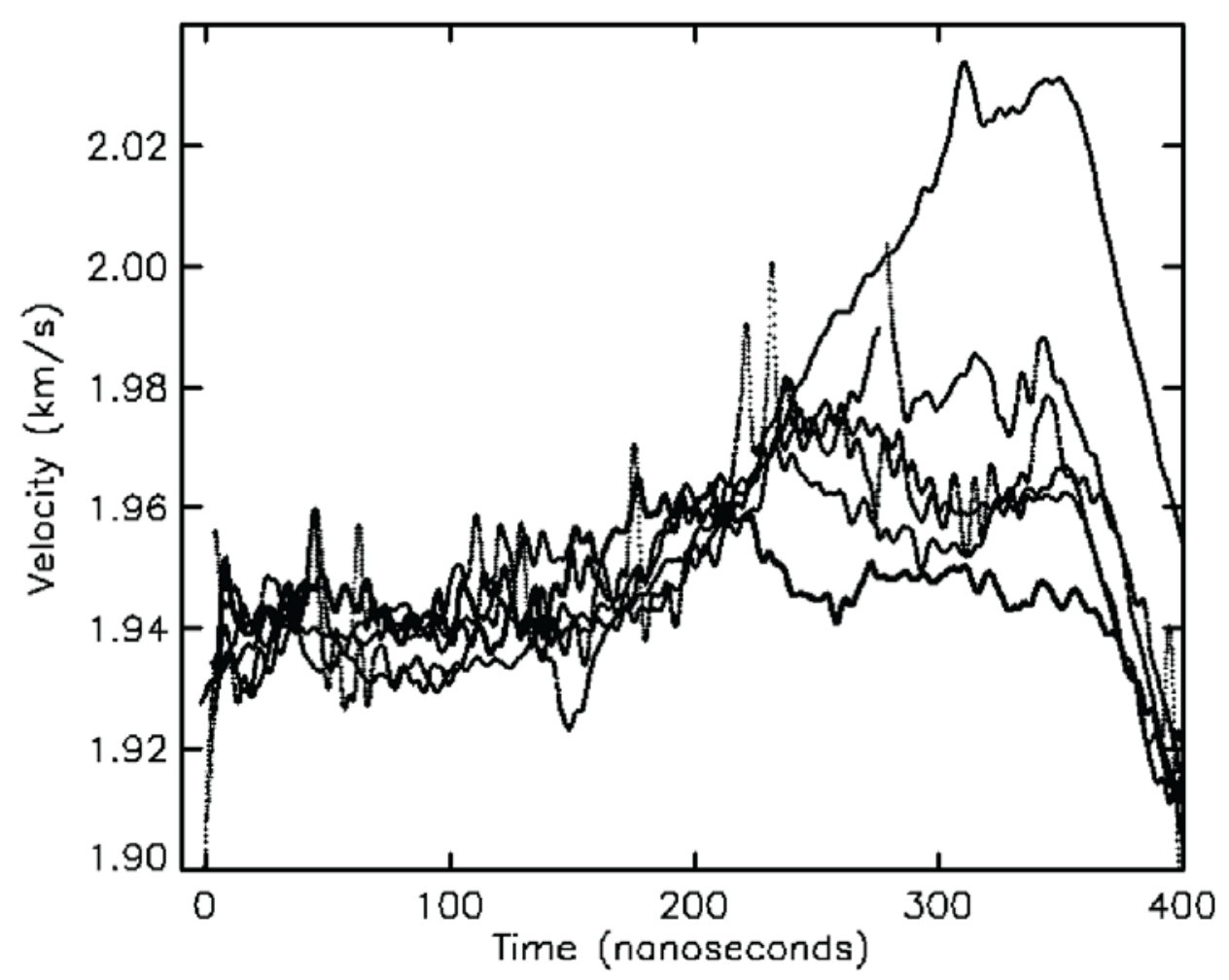

Figure 1.3.1 Example of PDV velocity data with data overlayed for six nominally identical channels. Overlaying identical channels indicates where unusual velocity deviations occur that do not follow the trends in the remaining "identical" channels. These data were obtained from a gas-gun shot using a flat-faced projectile launched with a two stage gas gun impacting a flat platinum sample backed by aluminum coated lithium fluoride. 

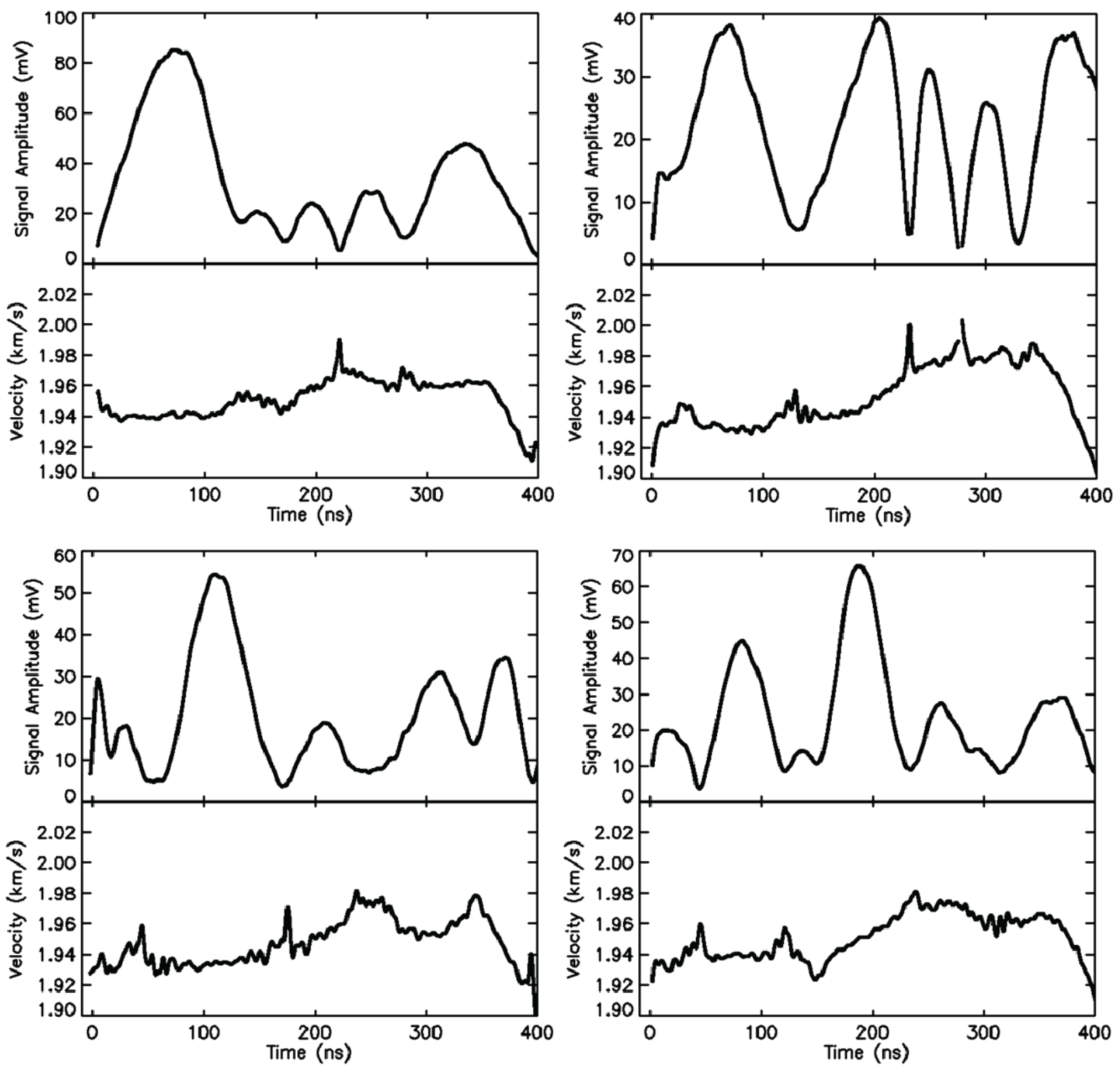

Figure 1.3.2. Four of the velocity traces in Figure. 1.3.1 with their associated signal amplitudes.

Four of the six traces in Figure 1.3.1 are shown separated in Figure 1.3.2 plotted with the amplitude of the oscillating-signal. The signal amplitude has amplitude modulation, which is not the same across identical channels. Based on similar, repeatable modulation with distance in slow speed bench measurements, this modulation is likely due to speckle effects from an average roughness of 0.2 micrometers of surface roughness applied to the LIF window before adding an aluminum coating. Because of random electronic and photon shot noise, signal to noise ratio is expected to be lower for lower amplitude. But there is another non-random effect that appears quite commonly when amplitude modulation is present: there are frequency deviations (spikes) larger than $1 \%$ in size in the velocity 
associated with the amplitude dips. We will see much later (Chapter 5 ) that similar spikes appear in simulations with roughness due to disruption of a delicate balance of path dependent frequency shifts through different points in the lens aperture. In the simulations, these spikes arise without the need to add additional random noise.

\subsection{Working hypothesis and plan for the derivation of instantaneous peak frequency for PDV}

Our working hypothesis for this derivation is that for a window width going to zero, $\tau \rightarrow 0$, there should be a unique value for the instantaneous peak frequency, and for least squares fitting or spectral averaging the peak should have the same value $\omega_{m}=\omega_{p}$. Since the instantaneous peak frequency is unique and since we are starting with a simulation with no noise, instead of trying to find the instantaneous peak frequency from a non-linear least squares fit we will identify the peak in the spectrum from the position where the derivative of the sideband spectral peak changes sign, i.e., where $\left.\frac{\partial P_{+}}{\partial t}\right|_{\omega_{p}}=0$

and take this in the limit $\tau \rightarrow 0$. We will show below that in a simulation without noise, the instantaneous peak $\omega_{p}$ exists, is defined, and has also the same value for the mean

$\omega_{m}=\langle\omega\rangle=\frac{\int_{B} d \omega \omega P_{+}}{\int_{B} d \omega P_{+}} \underset{\tau \rightarrow 0}{\longrightarrow} \omega_{p}$.

in the limit $\tau \rightarrow 0$. We will call the resulting peak frequency, $\omega_{p}$, the instantaneous peak frequency. We will derive $\omega_{p}$ in terms of time domain functions and understand this implicitly to be a function of time $\omega_{p}=\omega_{p}(t)$. Now we consider the physical origin of the time varying signals.

\subsection{A sideband spectral peak}

is connected with time varying difference in phases of scattered light and reference field

For the derivation of $\omega_{p}$, we will introduce a classical electromagnetic field description of a PDV experiment. In a PDV experiment, a source laser field $E_{\ell}$ with frequency $\omega_{\ell}$ is propagated towards a moving target. Light is scattered from the moving target. A scattered light field $E_{s}$ is collected by a lens and propagated to a detector. We incorporate point-wise light matter interactions into our picture. The total scattered light field at the detector will be a superposition of many individual scattered light fields at the detector 
$E_{s}=\sum_{i} E_{i}$

with individual scattered light fields $E_{i}$ labelled with index $i$. PDV is a heterodyne detection technique wherein a reference field $E_{r}$ is superposed at the detector, also, with amplitude similar to $E_{s}$. The total superposition of fields at the detector is a sum

$$
E_{r}+E_{s}=E_{r}+\sum_{i} E_{i}
$$

PDV experiments tend to be nearly one dimensional (paraxial) in the sense that the laser and optical system have small numerical aperture. We use a scalar approximation for transverse radiating optical fields wherein the magnetic fields are related to the electric fields as $B=E$ / $C$, and the detected optical power is proportional to $p \sim|E|^{2}+|B|^{2} \sim|E|^{2}=E^{*} E$, where * is the complex conjugate. The PDV signal is proportional to the detected optical power.

$$
p(t)=\left|E_{s}+E_{r}\right|^{2}=\left(E_{r}+E_{s}\right) \cdot\left(E_{r}+E_{s}\right) *=E_{r} \cdot E_{r} *+E_{s} \cdot E_{s}^{*}+E_{r} * \cdot E_{s}+E_{r} \cdot E_{s}^{*}=p_{r}+p_{s}+p_{+}+p_{-}
$$

(we do not write the proportionality constant and promise in advance that the proportionality constant will divide out of $\left.\omega_{p}\right) . \quad p_{r} \sim E_{r} \cdot E_{r} *$ is the optical power in the reference at the detector, which can be observed by blocking the scattered light. $p_{s} \sim E_{s} \cdot E_{s} *$ is the optical power in the scattered light. The optical interference in the detected power between the reference and scattered light spectra $p_{+}+p_{-}$creates an oscillating interference riding on a background $p_{r}+p_{s}$. The background can vary slowly also as the total scattered light intensity varies.

Let us introduce time into the complex fields $E_{r}(t)$ and $E_{s}(t)$, and note which parts of $p(t)=p_{r}+p_{s}+p_{+}+p_{-}$contains information on the motion of the target. In this work we will discuss only "conventional" PDV where the reference is derived from a stationary-partial reflection of the laser field, and $\omega_{\ell}=\omega_{r}$. Typically, the reference field at the detector has constant amplitude $\left|E_{r}\right|$, constant frequency $\omega_{r}$, and arbitrary phase $\varphi_{r 0}$, i.e., the reference is a harmonic function $E_{r}=E_{r 0} e^{-i \omega_{r} t}, E_{r 0}=\left|E_{r}\right| e^{-i \phi_{r 0}}$. The scattered light field $E_{s}$ has a narrow spectrum of $E_{i}$ with slightly differing frequencies and phases due to the motion of the laser from the point of view of the target, and the differing optical paths for scattered light back to the detector. (In Chapter 3 we will derive a scalar diffraction theory for a moving target and optical system and write $E_{s}(t)$ in the time domain.) For physically reasonable situations where the superposition of amplitudes and phases of the individual scattered light fields $E_{i}$ is equivalent to a single complex-valued function, $E_{s}$ can be written in the form $E_{s}=|A(t)| e^{i\left[\Phi(t)-\omega_{r} t\right]}$. Written in this way, the phase of $E_{s}$ is different from the reference phase by the 
amount $\Phi(t)$. There are two conjugate terms in the detected optical power $p_{+} \sim E_{r}^{*} \cdot E_{s}=\left|E_{r}\right||A(t)| e^{i \Phi(t)}$ and $p_{-} \sim E_{r} \cdot E_{s}^{*}=\left|E_{r}\right||A(t)| e^{-i \Phi(t)}$ that depend on the difference in phase (note $p_{+}=p_{-}{ }^{*}$ ). The scattered light amplitude $|A(t)|$ and phase difference $\Phi(t)$ both vary in time. From these considerations we expect the detected power and signal to have a constant DC offset when only the reference is present, $\left(E_{r} * E_{r}\right)=\left|E_{r}\right|^{2}$, a time varying background term that depends on the amplitude of the scattered light $\left(E_{s} * E_{s}\right)=|A(t)|^{2}$, and a real valued oscillating interference term similar to $2\left|E_{r}\right||A(t)| \cos (\Phi(t))$ (here we used $\left.p_{+}=p_{-}^{*}\right)$. The high frequency oscillations in the signal are represented in the function $\cos (\Phi(t))$, i.e., from an oscillating function of the phase difference $\Phi(t)$ advancing in time as the target moves. PDV is easiest to interpret when the amplitude of the scattered light $\sim|A(t)|$ (and it's associated background term $p_{s} \sim|A(t)|^{2}$ ) has a time variation that is slow compared to the high speed interference oscillations in $p_{+}+p_{-} \sim \cos (\Phi(t))$. The detected signal side bands $p_{ \pm}$are the parts of the signal that contain information on the motion of the target due to the shift in phase in the scattered light relative to the reference phase. (To save confusion later, the reader should note that detection of a narrow spectrum of harmonic functions determines a single function $\Phi(t)$ in the detected signal).

\subsection{A brief detour: The Doppler shift in one dimension}

\section{has two different contributions from space-like and time-like terms}

To motivate further our focus on the sideband spectrum in our analysis, we take a detour and develop the connection between the sideband spectrum and the motion of the target in one dimension (we develop a full three dimensional solution later). A few of the conventions and definition used later are established in this section.

In a step-and-integrate experiment, or at extraordinarily slow speeds that humans can sense (say, 0.1 $\mathrm{mm} / \mathrm{sec}$ ), one observes that the optical power oscillates through one period for a displacement very nearly equal to $\lambda / 2$-- optical interference is involved. The question then becomes what happens at higher speeds. At higher speeds one tends to discuss the problem in terms of rates of change with time. By timet, we mean the time ("now") that advances in the same way everywhere in the frame of reference of the laboratory and detector. Because of the propagation time delay $T$, light that arrives at a detector at time $t$ was scattered at an earlier light-scattering time $(t-T)$. Because the target is moving in time, the delay $T=T(t)$ also changes in time. For constant particle velocity $u_{p}$ and an initial position $x_{0}$, Newton told us the position of the target will be $x=x_{0}+u_{p} t$. But because of the optical delay time, the actual position at the scattering time $(t-T)$ was $x=x_{0}+u_{p}(t-T)$. Both depend on the same initial position, $x_{0}$. 
In the solution of wave equations, there are two possible choices $\pm\left(k x-\omega_{r} t\right)$ for the sign of the argument of a solution. In free space the speed of light $c$ is the ratio of $c=\omega_{r} / k$, where $k=2 \pi / \lambda$. Throughout this work, we represented the phase of a forward propagating plane wave field as $+\left(k x-\omega_{r} t\right)$. (This choice is not used by all authors. In particular Yariv uses the - sign and Goodman and Jackson use the + . This choice of phase is represented also in Fourier transforms, which due to symmetry also needs a choice of sign. We borrow the convention for the Fourier transform from Jackson, who consistently uses the same sign convention for both. Later we will borrow results for the Gaussian beam from Yariv, but will change the sign.) At the earlier scattering time $t-T$, an observer in the lab says a plane-wave laser would have had a phase value at the target determined by replacing $t \rightarrow t-T$ in the phase $+\left(k x-\omega_{r} t\right) \rightarrow\left(k\left(x_{0}+u_{p}(t-T)\right)-\omega_{r}(t-T)\right)$. Up to an unspecified constant phase, the difference in phases $\Phi(t)$ in the oscillating interference signal has these terms from the scattered light phase at the scattering time:

$\Phi(t)=\left\{\left[k x_{0}+\omega_{r} \beta(t-T)\right]-\omega_{r}(t-T)\right\}+\omega_{r} t$

Here we introduce the usual notation for a dimensionless velocity $\beta=u_{p} / c$. In this work, we will be concerned only with terrestrial velocities for which $\beta \stackrel{\sim}{<} 10^{-4}$. For the moment, we borrow a result that should seem heuristically plausible and is also derived below -- the instantaneous sideband peak

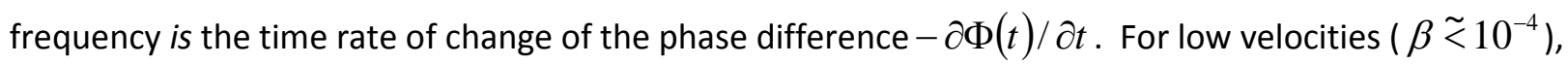
we may express the scattered light frequency as the laser frequency $\omega_{\ell}=\omega_{r}$ plus a small frequency shift $\omega_{s}$ :

$$
\omega_{s}=-\partial \Phi(t) / \partial t=-\omega_{r} \beta[1-\partial T / \partial t]+\omega_{r}[1-\partial T / \partial t]-\omega_{r}
$$

We will show later in three dimensions that there is a compact expression for the rate of change of delay time with time $\partial T$ / $\partial t$ that is of order of the relative velocity $\beta$ (times a direction-cosine factor). $\partial T / \partial t$ is precisely equal to $\beta$ in one-dimension. Since $\beta<<1$, the frequency shift $\omega_{s} \approx-\omega_{r} \beta[1-\beta]-\omega_{r} \beta \cong-2 \beta \omega_{r}$ to within a small relative error of order $\beta$. Here is it useful to note that the frequency shift has two distinct and dominant contributions, one term $-\omega_{r} \beta$ due to the changing shift in $k x$ from $x$ changing as $u_{p} t$, and one shift term $-\omega_{r} \partial T / \partial t=-\omega_{r} \beta$ due to the changing propagation delay back to the detector. We will show below that in a three-dimensional confocal arrangement (light out and back through the same lens and optical fiber) the Doppler shift $\omega_{s} \approx-2 \beta_{x} \omega_{r}=-2\left(u_{p x} / c\right) \omega_{r}$ is related to the component $u_{p x}$ of the particle velocity along the beam direction.

As an aside, we wish to make a comment related to the difference between a moving and stationary interference experiment. A stationary experiment may be performed by stepping the target position, stopping the motion, and integrating a measurement of the signal a "long time" after the step has 
stopped. In a step-stop-and-integrate experiment the phase differences in fields over different paths have time to readjust and the experiment "forgets" that it was ever somewhere else. The fields see a new initial position. In a high speed experiment, the fields have some memory of where they came from, i.e., they have some memory of initial positions at an earlier time. Due to the "reset" of initial positions, a step-stop-and-integrate interference experiment may be expected to give a different result than a moving experiment. At extremely low velocities $(<<1 \mathrm{~m} / \mathrm{s})$ used in Fourier Transform Spectroscopy with an incoherent source, this distinction may not be important. But for a high speed experiment we believe this memory of initial positions is a necessary element of a correct description of a PDV experiment where the laser field may have a coherence time longer than the experiment itself.

In a picture involving rates of change, the target senses a different time varying phase of the laser due to motion, and an extra time varying phase is introduced due to a changing delay for the scattered light. It is the combination of the rate of change of these two additional time varying phase shifts that is called the Doppler shift. The high speed oscillation at the Doppler shift frequency is the sideband spectral peak position in one dimension. This is why we are interested in the position of the peak in the sideband spectrum.

\section{CHAPTER 2. Instantaneous peak frequency}

\subsection{Relating the instantaneous signal frequency to the scattered light field - time domain picture}

Having seen the connection between the sideband spectral-peak position and the motion of the target in one dimension, we return to the problem of relating the instantaneous peak frequency $\omega_{p}$ to time dependent fields $E_{r}(t)$ and $E_{s}(t)$ in more than one dimension. We will explore the limitations of this idea later. For now, we are interested in how to relate the signal frequency to the phases of the fields.

There is a simple way to calculate $\omega_{p}$, and a rigorous method. In this section we look at the simple method. In later sections of this chapter, we look at the underlying implicit assumptions underlying the simple method.

A reasonable assumption (proved in section 2.2): for a simple experiment with one term in the detected optical power of the form

$2\left|E_{r}\right||A(t)| \cos (\Phi(t))$

the signal frequency is merely the time rate of change of the argument in the oscillatory factor $\omega_{p}=\frac{\partial \Phi(t)}{\partial t}$ 
We will have more to say about justifying this statement below. Later in this report (chapter 3) we will show a method of calculating $E_{s}=|A(t)| e^{i\left[\Phi(t)-\omega_{r} t\right]}$ and $E_{r}^{*} E_{s}=|A(t)| e^{i \Phi(t)}$ using diffraction theory for a moving target. The time rate of change of the product of the fields contains the desired term:

$$
\frac{\partial}{\partial t} E_{r}^{*} E_{s}=\frac{\partial}{\partial t}|A(t)| e^{i \Phi(t)}=\left\{\frac{1}{|A(t)|} \frac{\partial}{\partial t}|A(t)|+i \frac{\partial}{\partial t} \Phi(t)\right\}|A(t)| e^{i \Phi(t)}
$$

While $|A(t)| \neq 0$, we can divide by $E_{r}{ }^{*} E_{s}$ and take the imaginary part to pull out

$$
\omega_{p}=\frac{\partial \Phi(t)}{\partial t}=\operatorname{Im}\left[\frac{1}{E_{r}^{*} E_{s}} \frac{\partial}{\partial t} E_{r}^{*} E_{s}\right]
$$

A very common method of analysis of PDV data is to compute short-time spectra, and to take the signal frequency as the peak of the sideband spectrum. In section 2.2 , we venture into frequency space to see what assumptions are needed to obtain a similar relation between the peak frequency and the scattered light field.

\subsection{Relating instantaneous-sideband peak-frequency in a heterodyne-detection signal-spectrum}

\section{to time dependent optical fields - frequency domain picture}

For concreteness, we will use the Fourier transform and Dirac delta function defined in symmetric form by

$$
f(t)=\frac{1}{\sqrt{2 \pi}} \int_{-\infty}^{\infty} d t e^{-i \omega t} F(\omega), F(\omega)=\frac{1}{\sqrt{2 \pi}} \int_{-\infty}^{\infty} d t e^{i \omega t} f(t), \delta(p)=\frac{1}{2 \pi} \int_{-\infty}^{\infty} d q e^{i p q},\{p, q\} \in\{\omega, t\} .
$$

(We chose to use the Fourier transform in the derivation of $\omega_{p}$ for its convenient connection to the Dirac delta function $\delta(p))$. Figure 1.2.2 depicts the different parts of the spectrum $P(\omega)=P_{r}+P_{s}+P_{+}+P_{-}$which are related to terms in $p(t)=p_{r}+p_{s}+p_{+}+p_{-}$. The background consists of a delta function $P_{r}$ from the constant reference power, and a scattered light amplitude spectrum $P_{s}$ that straddles zero frequency. For experiments with uniform particle velocities $\vec{u}_{p}$, the signal sideband spectra $P_{+}$and $P_{-}$will have single, well-separated peaks with peak frequencies at $\pm \omega_{p}$ nearly proportional to $u_{p x}$.

Short time spectra are obtained by multiplying $p(t)$ by a peaked window function $g(t)$ that goes to zero for $|t|$ large. The short time spectrum $S(\omega)$ at time $t_{0}$ can be written in two ways by substituting the Fourier transforms for $g \Leftarrow G$ and $p \Leftarrow P$ 


$$
\begin{aligned}
& S(\omega)=\frac{1}{\sqrt{2 \pi}} \int_{-\infty}^{\infty} d t^{\prime} e^{i \omega t^{\prime}} g\left(t^{\prime}\right) p\left(t^{\prime}+t_{0}\right) \\
& =\frac{1}{\sqrt{2 \pi}} \int_{-\infty}^{\infty} d t^{\prime} e^{i \omega t^{\prime}}\left[\frac{1}{\sqrt{2 \pi}} \int_{-\infty}^{\infty} d \omega^{\prime} e^{-i \omega^{\prime} t^{\prime}} G\left(\omega^{\prime}\right)\right]\left[\frac{1}{\sqrt{2 \pi}} \int_{-\infty}^{\infty} d \omega^{\prime \prime} e^{-i \omega^{\prime \prime}\left(t^{\prime}+t_{0}\right)} P\left(\omega^{\prime \prime}\right)\right] \\
& =\frac{1}{\sqrt{2 \pi}} \int_{-\infty}^{\infty} d \omega^{\prime} G\left(\omega^{\prime}\right) \int_{-\infty}^{\infty} d \omega^{\prime \prime} P\left(\omega^{\prime \prime}\right) \frac{1}{2 \pi} \int_{-\infty}^{\infty} d t^{\prime} e^{-i \omega^{\prime \prime} t_{0}} e^{-i\left(\omega^{\prime \prime}+\omega^{\prime}-\omega\right)\left(t^{\prime}\right)} \\
& =\frac{1}{\sqrt{2 \pi}} \int_{-\infty}^{\infty} d \omega^{\prime \prime} G\left(\omega-\omega^{\prime \prime}\right) P\left(\omega^{\prime \prime}\right) e^{-i \omega^{\prime \prime} t_{0}}
\end{aligned}
$$

The last expression is obtained from the first after using the definition of the delta function. In the frequency domain, the short time spectrum $S(\omega)=S_{r}+S_{s}+S_{+}+S_{-}$is a convolution of $G$ and a spectrum of the time shifted $p=p_{r}+p_{s}+p_{+}+p_{-}$.

During PDV spectral analysis, one may compute real-valued amplitudes $|S|=\{S S *\}^{1 / 2}$ taken at a sequence of times (a spectral image) and search for trends of peaks as ridges in a frequency-time surface plot. For our simulation involving analytical functions with no noise, it is convenient to use the zero value of the derivative at the spectral peak to find the peak frequency. The same peak frequencies $\omega_{p}$ occur for the amplitude $|S|=\{S S *\}^{1 / 2}$ and or the signal-spectral power, $S S *$. To avoid working with the square roots, we may find $\omega_{p}$ for a simulated experiment by setting

$$
\left.\left.\frac{\partial}{\partial \omega}(S S *)\right|_{\omega_{p}} \equiv 0 \Rightarrow \operatorname{Re}\left\{S * \frac{\partial}{\partial \omega} S\right\}\right|_{\omega_{p}}=0
$$

A variety of short-time window functions are possible in spectral analysis. In this derivation for $\omega_{p}$ we use one possible choice with $g(t)$ selected to be a real-valued Gaussian function centered at $t=0$ with one standard deviation width $\tau$, and $g(t)=g(-t)$. During this derivation for the instantaneous peak frequency $\omega_{p}$ it is convenient that: the frequency transform $G(\omega)$ is a real-valued Gaussian centered at $\omega=0$ with width $1 / \tau$; that it's derivative $\partial G(\omega) / \partial \omega=-\tau^{2} \omega G(\omega)$ acquires a factor of $\omega$; that the transform of a product $\omega P(\omega)$ is a time derivative $i \partial p(t) / \partial t$; and that we can make the choice of $g(t)$ disappear from the result by taking $\tau \rightarrow 0$ with $g(t)$ taking on the properties of the Dirac delta function $g(t) \rightarrow \delta(t)$.

PDV spectral analysis is un-defined for sampled data and $\tau \rightarrow 0$. Once we take $\tau \rightarrow 0$, experimentally minded folks should note that intermediate expressions pass beyond applicability to PDV data analysis. The utility of this approach for deriving $\omega_{p}$ arises in simulations of the measured frequency. 
Taking the derivative of the signal-power spectrum $\partial\left(S S^{*}\right) /\left.\partial \omega\right|_{\omega_{p}}=0$ evaluated at $\omega_{p}$ we obtain an integral relation for $\omega_{p}$ :

$0=\operatorname{Re}\left\{\int_{-\infty}^{\infty} d \omega^{\prime \prime} G\left(\omega_{p}-\omega^{\prime \prime}\right) P^{*}\left(\omega^{\prime \prime}\right) e^{+i \omega^{\prime \prime} t_{0}} \int_{-\infty}^{\infty} d \omega^{\prime}\left(\omega_{p}-\omega^{\prime}\right) G\left(\omega_{p}-\omega^{\prime}\right) P\left(\omega^{\prime}\right) e^{-i \omega^{\prime} t_{0}}\right\}$

To avoid dividing by zero in the denominator during algebraic manipulations, we require a non-zero spectral-peak power in a region of interest (an easy requirement to satisfy, otherwise what is the point?):

$\int_{-\infty}^{\infty} d \omega^{\prime \prime} G\left(\omega_{p}-\omega^{\prime \prime}\right) P\left(\omega^{\prime \prime}\right) e^{-i \omega^{\prime \prime} t_{0}}=\sqrt{2 \pi} S\left(\omega_{p}\right) \neq 0$

Also note that for complex numbers $Z$ and $S, \operatorname{Re}[Z] / \operatorname{Re}[S * S]=\operatorname{Re}[Z] / S * S=\operatorname{Re}[Z / S * S]$. Rearranging, we arrive at an integral relation involving the peak frequency $\omega_{p}$ :

$\omega_{p}=\operatorname{Re}\left[\frac{\int_{-\infty}^{\infty} d \omega^{\prime} G\left(\omega_{p}-\omega^{\prime}\right) \omega^{\prime} P\left(\omega^{\prime}\right) e^{-i \omega^{\prime} t_{0}}}{\int_{-\infty}^{\infty} d \omega^{\prime} G\left(\omega_{p}-\omega^{\prime}\right) P\left(\omega^{\prime}\right) e^{-i \omega^{\prime} t_{0}}}\right]$.

Rushing to take the zero-width limit, we are halted on finding $\omega_{p} \rightarrow 0$ until we realize the delta function in the DC part of the reference $P_{r}$ captures the value for $\omega_{p}$. We are reminded that in PDV analysis and for our derivation of $\omega_{p}$ from diffraction theory of a moving target, it is useful to select a portion of the spectrum in a region of interest, and to ignore the remainder of the spectrum (especially the delta function at zero from the DC part of the light). For our present purpose, we imagine a region of interest defined by a real-valued box function $B(\omega)$ of unit amplitude with width $\Delta \omega$. The approach we will use to find a peak at $\omega_{p} \neq 0$ within the signal spectrum $P_{+}\left(\omega^{\prime}\right)$ is to select a region of interest function $B\left(\omega^{\prime}\right)$ with range selected to exclude the delta function from $P_{r}$ at $\omega=0$, and by replacing $P\left(\omega^{\prime}\right) \rightarrow B\left(\omega^{\prime}\right) P\left(\omega^{\prime}\right)$. For $P_{+}\left(\omega^{\prime}\right)$ narrow enough that it does not overlap $P_{r}$ (e.g., for a moving target), then $B\left(\omega^{\prime}\right) P\left(\omega^{\prime}\right)=P_{+}\left(\omega^{\prime}\right)+B\left(\omega^{\prime}\right) P_{s}\left(\omega^{\prime}\right)$. In the following, we will find we do not need to remove the residual overlapping term $B\left(\omega^{\prime}\right) P_{s}\left(\omega^{\prime}\right)$, but can use a trick to make it arbitrarily small.

The spectrum $\omega^{\prime} P\left(\omega^{\prime}\right)$ is the spectrum of the derivative $i \partial p(t) / \partial t$, which can be verified by

$$
\frac{\partial}{\partial t} p(t)=\frac{1}{\sqrt{2 \pi}} \int_{-\infty}^{\infty} d \omega^{\prime \prime} e^{-i \omega^{\prime \prime} t}\left(-i \omega^{\prime \prime}\right) P\left(\omega^{\prime \prime}\right), \quad \text { or } i \frac{\partial}{\partial t} p(t)=\frac{1}{\sqrt{2 \pi}} \int_{-\infty}^{\infty} d \omega^{\prime \prime} e^{-i \omega^{\prime \prime} t} \omega^{\prime \prime} P\left(\omega^{\prime \prime}\right)
$$


Substituting into the numerator of $\omega_{p}$ gives

$$
\begin{aligned}
\int_{-\infty}^{\infty} d \omega^{\prime} G\left(\omega_{p}-\omega^{\prime}\right) \omega^{\prime} P\left(\omega^{\prime}\right) e^{-i \omega^{\prime} t_{0}} & =\int_{-\infty}^{\infty} d \omega^{\prime}\left[\frac{1}{\sqrt{2 \pi}} \int_{-\infty}^{\infty} d t e^{i\left(\omega_{p}-\omega^{\prime}\right) t} g(t)\right]\left[\frac{1}{\sqrt{2 \pi}} \int_{-\infty}^{\infty} d t^{\prime} e^{i \omega^{\prime} t^{\prime}} i \frac{\partial}{\partial t^{\prime}} p\left(t^{\prime}\right)\right] e^{-i \omega^{\prime} t_{0}} \\
& =\int_{-\infty}^{\infty} d t e^{i \omega_{p} t} g(t) \int_{-\infty}^{\infty} d t^{\prime} i \frac{\partial}{\partial t^{\prime}} p\left(t^{\prime}\right) \frac{1}{2 \pi} \int_{-\infty}^{\infty} d \omega^{\prime} e^{i \omega^{\prime}\left(-t+t^{\prime}-t_{0}\right)} \\
& =\int_{-\infty}^{\infty} d t e^{i \omega_{p} t} g(t) i \frac{\partial}{\partial t} p\left(t+t_{0}\right)
\end{aligned}
$$

An expression for the denominator is obtained in similar fashion. Substituting back the Fourier transforms for $G \Leftrightarrow g, P_{+} \Leftrightarrow p_{+}, B P_{s} \Leftrightarrow q$ and $\omega P \Leftrightarrow i \partial p / \partial t$ relates $\omega_{p}$ to the time domain functions and their derivatives as

$$
\begin{aligned}
& \omega_{p}=-\operatorname{Im}\left[\frac{\int_{-\infty}^{\infty} d t^{\prime \prime} e^{i \omega_{p} t^{\prime \prime}} g\left(t^{\prime \prime}\right) \frac{\partial}{\partial t} p\left(t^{\prime \prime}+t_{0}\right)}{\int_{-\infty}^{\infty} d t^{\prime \prime} e^{i \omega_{p} t^{\prime \prime}} g\left(t^{\prime \prime}\right) p\left(t^{\prime \prime}+t_{0}\right)}\right] \\
& =-\operatorname{Im}\left[\frac{\int_{-\infty}^{\infty} d t^{\prime \prime} e^{i \omega_{p} t^{\prime \prime}} g\left(t^{\prime \prime}\right) \frac{\partial}{\partial t^{\prime \prime}}\left[p_{+}\left(t^{\prime \prime}+t_{0}\right)+q\left(t^{\prime \prime}+t_{0}\right)\right]}{\int_{-\infty}^{\infty} d t^{\prime \prime} e^{i \omega_{p} t^{\prime \prime}} g\left(t^{\prime \prime}\right)\left[p_{+}\left(t^{\prime \prime}+t_{0}\right)+q\left(t^{\prime \prime}+t_{0}\right)\right]}\right] \\
& =-\operatorname{Im}\left[\frac{\int_{-\infty}^{\infty} d t^{\prime \prime} e^{i \omega_{p} t^{\prime \prime}} g\left(t^{\prime \prime}\right) \frac{\partial}{\partial t^{\prime \prime}} p_{+}\left(t^{\prime \prime}+t_{0}\right)}{\int_{-\infty}^{\infty} d t^{\prime \prime} e^{i \omega_{p} t^{\prime \prime}} g\left(t^{\prime \prime}\right) p_{+}\left(t^{\prime \prime}+t_{0}\right)}\right]+O\left(\Delta \omega\left|A / E_{r}\right|\right)
\end{aligned}
$$

We changed the denominator and extracted an "error" term from the integral expressions in $\omega_{p}$ of $\operatorname{order} O\left(\Delta \omega\left|A / E_{r}\right|\right)$. The error term can be made arbitrarily small by letting the reference field $E_{r}$ be arbitrarily large compared to the amplitude of the scattered light $A$.

At this point we feel we need to make a distinction between experiments and simulations, since a real experimentalist's intuition should be uneasy about a zero value for $\left|A / E_{r}\right|$. Real optical detectors always have some maximum allowable optical power (a thermal limitation), and an optimum power (due to non-linearity on the way to full saturation). The reference power at a detector cannot be made arbitrarily large. Due to photon shot noise in the reference, electronic noise, and the minimum bit-size of a digital recorder, an experimentalist may see that taking $\left|A / E_{r}\right| \rightarrow 0$ as having no signal at all. But in a continuous simulation with no noise, there is no problem with having a nonzero scattered light 
amplitude and raising the reference to suppress the amplitude modulation spectrum $B\left(\omega^{\prime}\right) P_{s}\left(\omega^{\prime}\right)$ that leaks into the region of interest. After filtering $p_{+}$out of $p \rightarrow p_{+}$while ensuring $S_{+}\left(\omega_{p}\right) \neq 0$ and letting $\left|E_{r} / A\right|$ be arbitrarily large, we may take these expressions to the instantaneous limit $\tau \rightarrow 0$. In the limit $\tau \rightarrow 0, g(t) \rightarrow \delta(t)$. Fortunately this removes explicit dependence on $\omega_{p}$ inside the integrals $\left(e^{i \omega_{p} t^{\prime \prime}} \rightarrow 1\right)$. And we arrive at the desired result relating the instantaneous peak frequency to the time varying fields:

$$
\begin{aligned}
\omega_{p} & =-\operatorname{Im}\left[\left.\frac{1}{p_{+}\left(t_{0}\right)} \frac{\partial}{\partial t^{\prime}} p_{+}\left(t^{\prime}\right)\right|_{t^{\prime}=t_{0}}\right] \\
& =-\operatorname{Im}\left[\left.\frac{1}{\left(E_{r}^{*} E_{s}\right)} \frac{\partial}{\partial t}\left(E_{r}^{*} E_{s}\right)\right|_{t=t_{0}}\right]=\operatorname{Re}\left[\left.\frac{1}{\left(E_{r}^{*} E_{s}\right)} i \frac{\partial}{\partial t}\left(E_{r}^{*} E_{s}\right)\right|_{t=t_{0}}\right]
\end{aligned}
$$

The integrals over time are gone. Here, we arrive at an identity relation for the peak in the side band spectrum $\omega_{p}(t)$ based on the time domain function $E_{r}^{*}(t) E_{s}(t)$ arising from a cross term in the detected optical power and its time rate of change $\partial\left(E_{r}{ }^{*} E_{s}\right) / \partial t$. The arbitrarily large factor for the amplitude $\left|E_{r}\right|$ ratios out, but it is more compact to write this explicitly with a factor of $E_{r}^{*}$ for reasons that will become apparent much later when we take the time derivative inside an integral for $E_{s}$.

We claim without showing the details that an alternative derivation can be performed starting with an average $\omega_{m}=\langle\omega\rangle=\left(\int_{B} d \omega \omega S^{*} S\right) /\left(\int_{B} d \omega S^{*} S\right)$ instead of the frequency-derivative of the power spectrum. After substituting $S(\omega)$ from the integral expressions involving $g\left(t^{\prime}\right) p\left(t^{\prime}+t_{0}\right)$, one finds that taking the limit $\tau \rightarrow 0$ involves a products of delta functions. Generally, there are difficulties for defining the effect of products of delta functions in integrals. But for this particular derivation, one finds the limit $\tau \rightarrow 0$ can be defined when one takes the limit for the ratio of the numerator and denominator simultaneously (there is an extra factor of $\tau$ needed that is conveniently dealt with in the ratio). In the limit of narrow time window in the average, we obtain the same value $\omega_{m} \rightarrow \omega_{p}$. This supports our hypothesis that the value and expression for $\omega_{p}$ exists and is unique.

When $E_{r}^{*} E_{s}$ can be written in the form $E_{r}^{*} E_{s}=\left|E_{r}\right||A(t)| e^{i \Phi(t)}$, we find that $\omega_{p}$ is simply and precisely the value one might expect from a heuristic argument, $\omega_{p}=-\frac{\partial \Phi}{\partial t}$, 
i.e., that the instantaneous peak frequency is the time rate of change of the phase difference between the reference and the total scattered field. This is true without further approximation. In addition, these expression are applicable to any number of spatial dimensions (keeping in mind are working with a nearly one dimensional scalar approximation). The requirements for these expressions to be correct are $P_{+}$is zero for non-positive frequencies and for there to be a non-zero peak power $P_{+}\left(\omega_{p}\right) \neq 0$. This derivation does not depend on a constant particle velocity (although in simulations below we will satisfy partially the requirement of separable spectra by restricting $\vec{u}_{p}$ to be uniform and constant).

The utility of an expression for the instantaneous sideband peak frequency $\omega_{p}$ written in terms of a product of time dependent fields $E_{r}(t)^{*} E_{s}(t)$ is at least two fold. These expressions are not applicable to PDV data analysis. The real power of these relations will become apparent when we can relate $E_{s}$ to an experiment configuration through diffraction theory (Chapter 3). After developing and incorporating an expression for $E_{s}$ (Chapter 4), we gain insights into what PDV measures (Sections 4.2-4.4). Additional utility of analytic integrand expressions involving time dependent $\left[E_{r}^{*} E_{s}\right](t)$ in three dimensions arises later in considerations related to efficiency of computation of $E_{s}(t)$. For example, instead of estimating $\omega_{p}=-\partial \Phi / \partial t$ from the phase of the complete integral at two closely spaced time steps, we may bring the time derivative inside the analytical expression for the integral $E_{r}{ }^{*} E_{s}$ and write down an algebraic expression for the derivative before numerical integration. Using this approach requires integration only over space (target coordinates and aperture coordinates) at one time step to obtain $\omega_{p}=-\partial \Phi / \partial t$ (which provides some increase in computational efficiency).

\subsection{The instantaneous peak frequency is equivalent to the real part of a sum involving a delicate balance of complex-valued weights (i.e., not a statistical average)}

A decomposition of $E_{s}$ into a spectrum of harmonic functions provides an insight into the relationship of $\omega_{p}$ to an underlying spectrum of Doppler shifts. From the perspective of the laser source, the laser field is a function of space and time $E_{\ell}(\vec{r}) \operatorname{Exp}\left(i \phi_{\ell}(\vec{r}, t)\right)$. For a continuous wave laser with phase $\phi_{\ell}(\vec{r}, t)$ advancing linearly in time in the same manner everywhere, its spectrum is a delta function, i.e., is harmonic. From the target's perspective, the laser source is moving, and the field acquires some amplitude modulation. Also, the delay $T=T(t)$ between a scattering event and the detector depends on a path between scattering event and detector, and is slightly non-linear in time for any individual path (we will write down a closed form expression for this in section 3.5). As a consequence, the phase in individual scattered fields $E_{i}$ does not advance strictly linearly, and $E_{i}$ is not perfectly harmonic, i.e., 
is not purely of the form $\left|E_{i}\right| e^{-i\left(\omega_{i}+\omega_{r}\right) t}$ with phase $\left(\omega_{i}+\omega_{r}\right) t$ advancing linearly in time. The total phase difference $\Phi(t)$ is slightly nonlinear.

On the other hand, any reasonable field (satisfying Maxwell's equations) can be expressed as a superposition of harmonic fields using Fourier transforms (we review these ideas in section 3.3.1). In principle, it should be possible to fully transform each of the individual time dependent scattered fields at the detector into a spectrum of harmonic functions

$$
E_{i}=\sum_{j} E_{i j}=\sum_{j}\left|E_{i j}\right| e^{-i \varphi_{i j}} e^{-i\left(\omega_{i j}+\omega_{r}\right) t},
$$

where $e^{-i \varphi_{i j}}$ are initial phase factors, and phases $\left(\omega_{i j}+\omega_{r}\right) t$ advance linearly. The total scattered field is a sum of harmonic functions

$$
E_{s}=\sum_{i} E_{i}=\sum_{i, j}\left|E_{i j}\right| e^{-i \varphi_{i j}} e^{-i\left(\omega_{i j}+\omega_{r}\right) t} .
$$

Expanded in harmonic functions, the individual scattered fields $E_{i}$ consist of a spectrum of frequencies $\omega_{i j}+\omega_{r}$ written as shifts $\omega_{i j}$ that are small compared to $\omega_{r}$ with initial phases $\varphi_{i j}$. With the scattered field ultimately expanding to a spectrum of harmonic fields we would find that the instantaneous sideband peak frequency $\omega_{p}$ takes the form

$$
\omega_{p}=\operatorname{Re}\left[\left.\frac{1}{\left(E_{r}^{*} E_{s}\right)} i \frac{\partial}{\partial t}\left(E_{r}^{*} E_{s}\right)\right|_{t=t_{0}}\right]=\operatorname{Re}\left[\frac{\sum_{i, j} \omega_{i j} E_{i j}}{\sum_{i, j} E_{i j}}\right]=\sum_{i, j} \omega_{i j} \operatorname{Re}\left[\frac{E_{i j}}{\sum_{i, j} E_{i j}}\right]
$$

This form for the peak in the signal spectrum $\omega_{p}$ arises as a consequence of using a heterodyning technique with optical interference between two different fields, one of which as a narrow spectrum. The expression has similarities to an average over the spectral content $\omega_{i j}$ with weights $E_{i j}$ from individual scattered fields. Notice that the derivation of this expression was in a classical limit of high reference-optical power and the averaging should not be confused with a statistical average. Also, the "weights", $E_{i j}$, in the "average" are not real positive probabilities such as $\left|E_{i j}\right|^{2}$. Even after taking the real part of $E_{i j} / \sum_{i, j} E_{i j}$ the peak frequency $\omega_{p}$ has positive and negative contributions $\omega_{i j} \operatorname{Re}\left(E_{i j} / \sum_{i, j} E_{i j}\right)$ depending on the phases of $E_{i j}$. In this "weighted average," there is a possibility of cancellations between terms with similar amplitude and opposite sign. These cancellations arise in a manner similar to "interference" during the detection of the optical powers, but strictly speaking we 
may not want to confuse these cancellations directly with "optical interference" because this is not an expression for an optical power.

In the next chapter, we will write down $E_{i}=E_{i}(t)$ using an approximation from diffraction theory developed for an optical system and moving target. For reasons of computational convenience, we will not carry out the transform to a sum of harmonic functions. But because $E_{i}(t)$ can be nearly harmonic we will find that under special limiting circumstances that the expressions have similarities to this average over harmonic functions. And we will see how for nearly symmetric configurations (Sections 4.2 and 4.3 ), that cancellations take place in a delicate balancing act that can be slightly disrupted by small "irregularities" (chapter 5).

\section{CHAPTER 3. The detected field}

\subsection{Classical electromagnetic wave propagation and point-wise matter-field scattering}

A target moves in a laser field. Laser light is scattered from the target. The scattered field is collected and returned to a detector and mixed with a reference field. A detected signal is generated.

In this chapter, we develop a diffraction theory adapted for a lens-based probe and for a moving target. We will make approximations. The theory is not exact. We believe the approximations allow insights into how PDV works.

We derive approximate expressions for the detected portion of the total time-dependent scattered-light field based on diffraction theory. We include target motion and lenses. Near the end of this chapter, we write down the instantaneous peak frequency (see section 2.1 and 2.2) in terms of the time dependent fields. We explore the similarities of these expressions to the expression for a complete expansion in harmonic functions from the last chapter (section 2.3). This approximation allows insights into the essential elements needed to simulate the field and instantaneous peak frequency in a PDV experiment. We will show later how to use this derivation in numerical calculations of the side-band peak frequency with and without surface roughness (chapter 5 ).

We will work with results from classical electrodynamics needed for wave propagation and diffraction theory. For the superposition of waves after a "reflection" from a moving target, we borrow inspiration from the foundations of quantum mechanics related to the "wave-particle" duality: Light propagates in the available space as a wave. When light encounters matter, light-matter interactions occur at pointsthese interactions are discrete in time, countable, and localized.

Point-wise matter-field interactions should not be surprising and the reader familiar with optical detection has seen it's effects. The underlying particle nature of discrete-photon detection can be 
discerned by observing the noise in a detector signal with the light turned on or off. Photon shot noise is an indication that photons are detected discretely and carry an element of Poissonian probability for the detection of a mean number of photons per unit time. In our derivation for PDV scattered light, we will be interested in the side-band peak frequency for the "mean" detected power in a classical (average) sense. In this derivation we will ignore detection shot noise and work in the classical limit where power is raised high enough that we can ignore shot-noise fluctuations relative to the mean value. This means we will not be sensitive to the idea that energy is carried as lumps called photons while propagating as a wave. But we believe it is essential to keep the notion of spatially localized mater-field interactions to reflect what actually happens in a PDV measurement.

On its face, the following derivation is based on classical electrodynamics, classical wave optics, and scalar diffraction theories (and includes ideas consistent with relativistic electrodynamics arguments). But to return light, we need to include an "optical reflection" from a moving target. We regard the reflection as a series of point-like "scattering events" and think of this as related to discrete point-like interactions. By "point-wise," we will mean that the interaction between charges in the target and the field occurs at points in space and time, and a "new" field is generated and propagates away as if generated by a collection of moving point sources. By "coherent" we will mean the scattered light from each scattering point has a definite-phase relationship to the phase of the source field at the point where the scattering occurs, and that we sum amplitudes before detecting the classical power in the field. Using wave optics and including coherent point-wise discreteness in the "reflection" we will obtain a result consistent with phenomena observed in PDV: PDV appears to measure precisely the component of the velocity along the beam direction, $\omega_{p}=2 \omega_{r} \beta_{x}$. Using a three dimensional field theory, we gain an additional insight that the heterodyne signal frequency involves a delicate balance of contributions from a narrow distribution of frequency values with complex-valued weights that depend on the propagation phases. Under circumstances where the paths followed to the detector involve large angles away from the optic axis (for example when using a large lens), the distribution of frequencies can have a width on the order of $1 \%$.

\subsection{Setting up a field calculation for a flat surface traveling at constant uniform velocity} with confocal illumination, and observation using a probe with lens and optical fiber

The following diagram 3.2.1 is an abstraction of quantities of interest in a three dimensional PDV experiment with a lensed probe. For a derivation of the detected field, we are interested in: an optical fiber with end-face center at point $P_{0}$; a "probe body" consisting of an opaque screen with aperture $A$; two closely spaced "thin" lenses in the aperture with position-dependent phase delays $k \delta_{f}$ and $k \delta$; and a moving target traveling with particle velocity $\vec{u}_{p}$. We may occasionally refer to the left lens labelled with phase delay $k \delta_{f}$ as the fiber lens, and right lens with phase delay $k \delta$ as the target lens. 


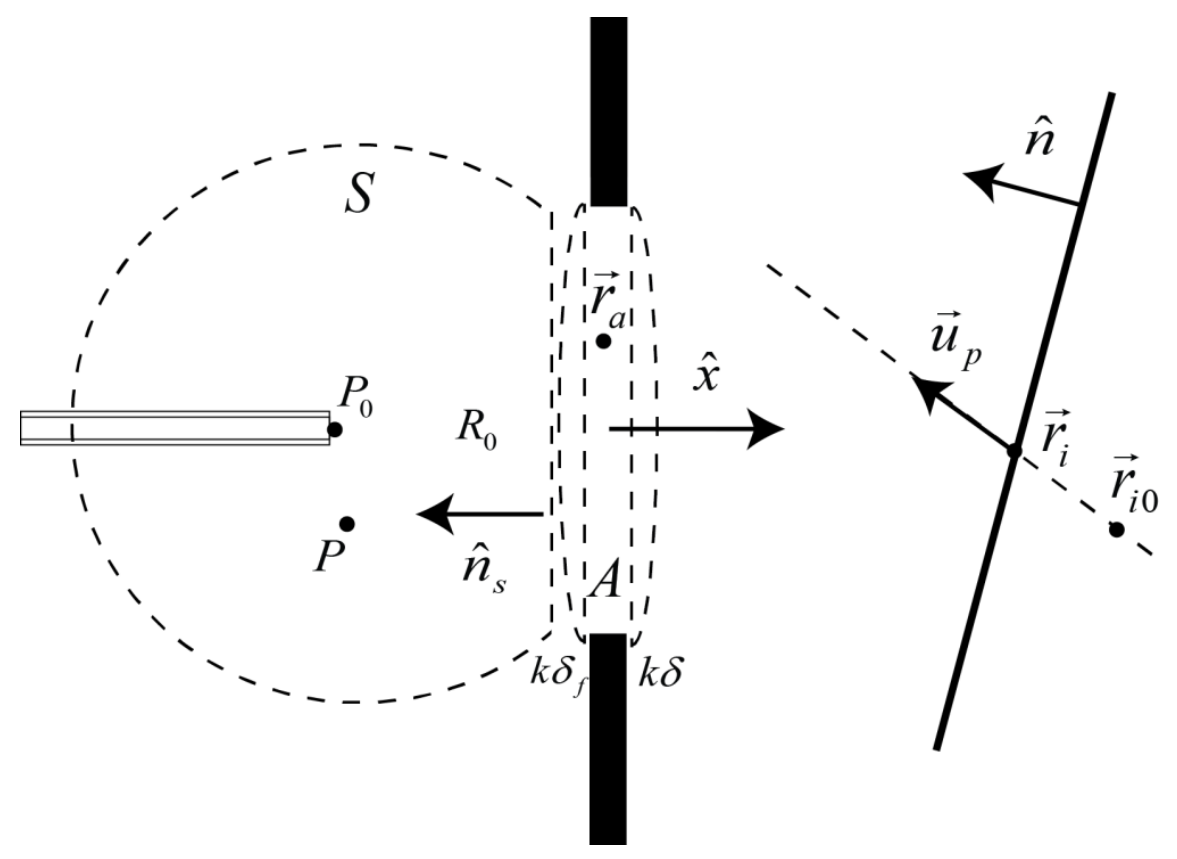

Figure 3.2.1. Abstraction of a PDV confocal-probe experiment configuration to be used in a diffraction calculation.

We describe the target classically as a set of points labeled with an indexi (in a very loose sense we may think of $i$ as a particle summation index). We now restrict this discussion to the case where all target points move with uniform constant particle velocity $\vec{u}_{p}$. At some earlier time $t=0$, the set of target points had initial positions $\vec{r}_{i 0}$. With constant uniform particle velocity, all the target points move along parallel straight lines described by $\vec{r}_{i}=\vec{r}_{i 0}+t \vec{u}_{p}$.

The lenses are centered on the fiber axis. The lenses and fiber define an optical axis. In the diagram we label the optic axis with direction $\hat{x}$. Later, we will imagine a coordinate origin in the aperture plane with two additional orthogonal directions $\hat{y}$ and $\hat{z}$ in the plane of the aperture (not shown).

The laser field (not shown) emerges from the fiber. The lenses cause the laser field to converge and diverge with symmetry axis along the optical axis $\hat{x}$. We may sometimes refer colloquially to a "laser beam direction." Due to curvature of phase fronts in a converging-diverging Gaussian beam the power in the field does not propagate strictly parallel to $\hat{x}$, but it is symmetric about optical axis $\hat{x}$.

Since we will use a point-wise scattering description, and since the motion of the target is described by the motion of points $\vec{r}_{i}=\vec{r}_{i 0}+t \vec{u}_{p}$, one way to describe the detected-field superposition is a sum of fields scattered from source points traveling along lines, $\vec{r}_{i}=\vec{r}_{i 0}+t \vec{u}_{p}$, with appropriate time retardation and scattering amplitude derived from the amplitude of the laser field.

We consider the set of target particles to be reflective and "opaque" on a length scale much smaller than the wavelength - we ignore multiple scattering. A flat, opaque and reflective surface with a short skin depth much shorter than the wavelength is an example of a target that does not need to be 
described by multiple scattering. The initial positions of points in the target are labelled $\vec{r}_{i 0}$. We allow the orientation of a flat target surface to be arbitrary and described by surface normal $\hat{n}$. We will connect $\hat{n}$ with the $\vec{r}_{i 0}$ description later. In this derivation, we consider the velocity $\vec{u}_{p}$ and targetsurface normal $\hat{n}$ to be arbitrary and independent of the optical axis $\hat{x}$.

Real lenses are finite in extent. Lenses must be held by some device (a metal tube, for example), which also prevents light from going around the lenses. For an absorbing enclosure surrounding the aperture and fiber, the shape of the enclosure is irrelevant. We represent the lens tube as a completely absorbing opaque and flat barrier with transparent aperture $A$. For mathematical convenience, we will approximate the lenses as infinitesimally thin and separated an infinitesimal distance from the aperture (for this approximate calculation we will take the lenses to be "in" the aperture in section 3.3.2).

Two lenses are equivalent to one lens by summing the optical delays. One reason for introducing two closely spaced lenses is the convenience of being able to arrange independent functional forms for $k \delta$ and $k \delta_{f}$ with independent focal distances. For efficient light collection, we may arrange for the fiberend face to be positioned "at the focal distance" $R_{0}$ from the fiber lens $k \delta_{f}$ (the mathematically convenient consequences of placing the fiber at the focus of one of the lenses will become apparent at the end of section 3.3.2.).

Laser light is scattered at points labelled $i$. Scattered light propagates in all accessible directions. Due to the opaque screen (lens holder), the field at the fiber arises from a superposition of scattered light that "fills" the aperture and lenses and propagates to the fiber. A part of the scattered light field that "overlaps" a guided-field mode in the plane of the fiber end face is coupled into and guided back to the detector on the fiber. On its way to the collection fiber, the scattered light field need not resemble the narrower laser beam (the lens-aperture size may affect the PDV signal).

Later we will add a small amount of static roughness to the target surface. We will add roughness to the initial positions $\vec{r}_{i 0}$ of a flat, tilted surface with normal $\hat{n}$. We will ignore multiple scattering as this adds another layer of "random" but static phase shifts, and we will consider this to be part of the roughness. In chapter 5 we will show examples with and without roughness and the effects of roughness combined with tilt on the summation of field phases.

\subsection{Diffraction of the aperture field at the fiber}

In this section, we consider how the field at points $P$ near the fiber are related to the scattered fields in the aperture $A$. Later we will return to the task of writing down how the fields scattered from the target are propagated to the aperture (section 3.4). This will set the stage for how we include retardedtime dependence in the expression for the fields that we will use to calculate the instantaneous frequency. For lens not too large and target not close to the lens, PDV light propagation is paraxial, or 
rather very nearly along the optical axis. The fields are very nearly transverse to the optical axis. We approximate the detected field using a scalar value for the transverse field.

\subsubsection{Review of scalar diffraction theory}

It is useful to review notions related to classical scalar wave propagation and diffraction from Born \& Wolf, Goodman, and Jackson. The reason for considering a scalar theory is that PDV fields are nearly transverse to the optic axis. We need only consider the transverse scalar component. Scalar components of an electromagnetic field propagate while satisfying the wave equation:

$c^{2} \nabla^{2} E=\partial^{2} E / \partial t^{2}$. A physically reasonable field component $E$ can be written as a superposition of harmonic fields (each having a phase advancing linearly as $\omega t$ ): $E=(1 / \sqrt{2 \pi}) \int_{-\infty}^{\infty} d \omega U_{\omega} e^{-i \omega t}$. The spectrum of harmonic functions $U_{\omega}=(1 / \sqrt{2 \pi}) \int_{-\infty}^{\infty} d \omega E e^{i \omega t}$ are functions of space. By expanding in frequency components $U_{\omega}$, the time dependence drops out of the wave equation and the $U_{\omega}$ components satisfy the Helmholtz equation, $\nabla^{2} U_{\omega}+k^{2} U_{\omega}=0$, where $\omega=k c$. Green's second theorem for fields relates a volume integral to an enclosing-surface integral. A Green's function in two vectors $\vec{X}^{\prime}$ and $\vec{X}$ that satisfies $\nabla^{2} G+k^{2} G=-\delta\left(\vec{X}^{\prime}-\vec{x}\right)$ can be used to convert Green's second theorem to a relation between a closed-surface integral and a point inside. Consider the closed surface $S$ and internal points $P$ (Figure 3.2.1). (In some places we will write functions as having a symbolic dependence on quantities labelled $A, S, P$ without reference to a coordinate frame). Kirchhoff showed that for a Green's function $G(S, P)$ and harmonic field $U_{\omega}(S)$ satisfying Helmholtz's equation on the surface $S$, the field $U_{\omega}(P)$ everywhere inside the enclosure at points $P$ is determined by an integral on the enclosure surface $S$ involving gradients of $U_{\omega}(S)$ and $G(S, P)$ :

$U_{\omega}(P)=\iint_{S} d S\left[U_{\omega}(S)\left(\hat{n}_{S} \cdot \vec{\nabla}_{S} G(S, P)\right)-G(S, P)\left(\hat{n}_{S} \cdot \vec{\nabla}_{S} U_{\omega}(S)\right)\right]$

(see for example Born and Wolf, Goodman, or Jackson). The gradient $\hat{n}_{S} \cdot \vec{\nabla}_{S}$ is taken in the direction $\hat{n}_{S}$ normal to the enclosure $S$ as shown in the Fig. 3.2.1. Functions $H$ that satisfy a Helmholtz equation $\nabla^{2} H+k^{2} H=0$ can be added to a particular Green's function which creates a family of Green's functions $G+H$. An example of $U_{\omega}$ or $H$ is a harmonic field that propagates outwards from a point as a spherical wave proportional to $e^{i(k r-\omega t)} / r$, who's amplitude decreases inversely with distance, $1 / r$, and phase $k r-\omega t=k(r-c t)$ has the same value for a sphere expanding outwards with $r=c t$. Rayleigh and Sommerfeld exploited the fact that the Green's function in the expression above is actually any one of a family of solutions $G+H$ and used this freedom to adjust $G(S, P)=0$ on the enclosure surface (the term with $\hat{n}_{S} \cdot \vec{\nabla}_{S} U_{\omega}(S)$ drops from the integral expression leaving the $U_{\omega}(S)$ term). They allowed the gradient $\vec{\nabla}_{S} G$ to be non-zero (otherwise the other term drops, and there is no field 
$U_{\omega}(P)$ inside; Rayleigh and Sommerfeld corrected this inconsistency in the original formulation by Fresnel and Kirchhoff. See Jackson or Goodman for a discussion of this problem. The author believes the often revered Born and Wolf are not clear on this point.). For convenience, we imagine that the space between the lens aperture and fiber is enclosed inside absorbing walls so that $U_{\omega}(S)=0$ at the inner surfaces of the optical probe, except in the lens aperture (the problem of our "lens tube" not being the same as an "aperture plate" is removed by black walls).

For the moment we imagine that the remaining non-zero part of $U_{\omega}(S)$ in the aperture is known. We will see below when calculating this scattered light field from a moving target the fields at the aperture are not harmonic (see discussion in section 3.4 including the idea that the target sees the spatially dependent laser field from a moving source), i.e., that they have a spectrum of frequencies that varies in time. And we will need to relate the time varying fields with a spectrum on the aperture to the field at the fiber. By transforming a spectrum of harmonic functions back to time dependent fields, we can write the time dependent solution near the fiber as

$$
E(P, t)=(1 / \sqrt{2 \pi}) \int_{-\infty}^{\infty} d \omega \quad U_{\omega} e^{-i \omega t}=\iint_{S} d S \frac{1}{\sqrt{2 \pi}} \int_{-\infty}^{\infty} d \omega e^{-i \omega t}\left[U_{\omega}(S)\left(\hat{n}_{S} \cdot \vec{\nabla}_{S} G(S, P)\right)\right]
$$

The reader is referred to Jackson's expression 9.130 for Dirichlet's form for $G$ near a flat-plane that limits to $G(S, P)=0$ on $S . G(S, P)$ can be arranged to satisfy the Green's and Helmholtz equations and satisfy the boundary condition $G(S, P)=0$ in the aperture with two spherical-wave like terms reflected about the aperture plane that cancel in the aperture (also known as the method of images). The gradient in the aperture is non-zero and becomes

$\hat{n}_{S} \cdot \vec{\nabla}_{S} G(S, P)=-\frac{1}{2 \pi} R_{0} \frac{e^{i k R_{P S}}}{R_{P S}^{3}}\left[1-2 i k R_{P S}\right]$.

Now there are two terms. Note that the gradient of $G(S, P)$ pulls a frequency and wavelength dependent factor $i k=i \omega / c$ from the phase factor down into the amplitude for one of the terms. For the usual configuration with $k R_{P S} \gg>1$, the amplitude of the wavelength dependent term is large.

\subsubsection{Including the fiber lens in the aperture-fiber diffraction calculation}

As a convenient approximation, we will take the lenses to be infinitesimally thin. The fiber-lens phase delay $k \delta_{f}=k \delta_{f}\left(\vec{r}_{a}\right)$ depends on points in the aperture $\vec{r}_{a}$. We regard the apparent thickness, $\delta_{f}\left(\vec{r}_{a}\right)$, as being due to an index of refraction variation and ignore the actual thickness. Next, we imagine a limit where the enclosure $S$ and thin lenses labelled $k \delta$ and $k \delta_{f}$ all coalesce into the aperture plane $A$ and occupy the same points $\vec{r}_{a}$ in the aperture. In this limit, the field on the enclosure $S$ is related to the field in the aperture $A$ by the phase shift introduced by the fiber lens, 
$U_{\omega}(S)=U_{\omega}(A) e^{i k \delta_{f}}$

Next we examine the field near the fiber. A single mode optical fiber has core diameter on the order of several wavelengths (PDV laser wavelengths are typically 1.55 microns, and SMF-28e fiber has a core diameter of 9 microns). For aperture-to-fiber distance $R_{o}$ that is several times larger than the aperture diameter, the spatial variation of the field at the fiber does not change rapidly as we change the observation point $P$ near $P_{0}$. For the coupling of scattered fields into the core, we will use another approximation - we will take the amplitude of the guided mode to be proportional to the field at the point $P_{0}$ and replace $E(P, t) \rightarrow E\left(P_{0}, t\right)$. (There is a weakness in the present approximation for rays with large angles into the fiber - see the discussion on this point in section 3.3.3). Because the field is zero everywhere on $S$ accept for points in the aperture $A$, the remaining nonzero part of the integral of the scattered light fields over the aperture area becomes

$E\left(P_{o}, t\right)=\iint_{A} d A \frac{1}{\sqrt{2 \pi}} \int_{-\infty}^{\infty} d \omega e^{-i \omega t}\left[U_{\omega}(A) \frac{-1}{2 \pi} R_{o} \frac{e^{i\left(k R_{P A}+k \delta_{f}\right)}}{R_{P_{o} A}^{3}}\left[1-2 i k R_{P_{o} A}\right]\right]$

$R_{P_{o} A}$ is the distance from points $\vec{r}_{a}$ in the aperture to the fiber point $P_{o}$. The phase delay $k R_{P_{o} A}$ between points in the aperture and the fiber increases for $\left|\vec{r}_{a}\right|$ increasing away from the optical axis.

A requirement for imaging between a pair of stationary points is the phase delays add constructively for all paths between the pair and through a lens -- constructive interference is satisfied if the lens delay compensates for the path length differences. For this work, we choose the fiber $P_{o}$ to be "at the focus of the fiber lens." The conjugate image position is at infinity. Since the delays on the infinite side are all the same, placing the fiber at the focus of the fiber lens is equivalent to choosing the same value $k R_{f} \equiv k \delta_{f}\left(\vec{r}_{a}\right)+k R_{P_{o} A}\left(\vec{r}_{a}\right)$ for all points $\vec{r}_{a}$. Notice that while the total phase $k R_{f}$ is the same for all aperture points, this value does vary with the wavelength. But here is a trick we use: for the infinitefocus arrangement to one point, the time delay between all points in the aperture to the fiber point $P_{o}$ has same value $R_{f} / C$.

There are two frequency and wavelength dependent phase factors in the integral: one from the frequency-time transform $e^{-i \omega t}$, and the aperture-point independent value $e^{i\left(k R_{f}\right)}$. In free space, $\omega=k c,-\omega t+k R_{f}=-\omega\left(t-R_{f} / c\right)$, and the phase factors combine to give $E\left(P_{o}, t\right)=\frac{-1}{2 \pi} R_{o} \iint_{A} d A \frac{1}{R_{P_{o} A}^{2}} \frac{1}{\sqrt{2 \pi}} \int_{-\infty}^{\infty} d \omega e^{-i \omega\left(t-R_{f} / c\right)}\left[\frac{1}{R_{P_{o} A}}-2 i \frac{\omega}{c}\right] U_{\omega}(A)$ 
Below, we will note that the scattered light field is not strictly harmonic, and it will be convenient to work with the time domain fields and may not be convenient to transform the scattered light to the spectrum $U_{\omega}(A)$. We do this also because we want a time dependent field for the instantaneous frequency calculation. By taking a time derivative of a Fourier transform integral one may verify that the transform of $\omega U_{\omega}$ is $i \partial E / \partial t$. We return to the time domain by substituting the transforms $U_{\omega} \Leftrightarrow E$ and $\omega U_{\omega} \Leftrightarrow i \partial E / \partial t$. With lens defined to provide position independent phase delay to $P_{o}, E\left(P_{o}, t\right)$ at the fiber is related to an integral of $E\left(A, t-R_{f} / C\right)$ in the aperture at earlier time $t-R_{f} / c$ :

$E\left(P_{o}, t\right)=\frac{-1}{2 \pi} R_{o} \iint_{A} d A \frac{1}{R_{P_{o} A}^{2}}\left\{\frac{1}{R_{P_{o} A}}+2 \frac{1}{c} \frac{\partial}{\partial t}\right\} E\left(A, t-\frac{R_{f}}{c}\right)$

Inserting the infinite-focus fiber lens has these unusual effects: The field $E$ incident on an aperture $A$ with spectrum $U_{\omega}$ propagates after delay $R_{f} / C$ to the point $P_{o}$. We call this the detected field. And with our focusing lens in place to collect all the fields on the aperture and focus them on the fiber, the detected field is a superposition of field components $E\left(A, t-R_{f} / c\right)$ that arrived simultaneously at the aperture.

But this expression says there is a second field that depends on the time derivative of the field in the aperture. Why? In order for the absorbing screen to "stop" the incident field, this is equivalent to the screen "emitting" another field. It seems oddly remarkable that with the lens in place this "extra" field needed to cancel the incident field on the absorbing screen outside the lens aperture is exactly the time derivative of the field summed over points inside the aperture. Also, since $E$ oscillates at a rate close to $\omega_{r}, \omega_{r} / c=2 \pi / \lambda$, and since $R_{P_{o} A}>>\lambda$, it will turn out below that the summation of the time derivative of the field over the aperture is more important than summation of the field itself in the aperture (an unavoidable conclusion is strangely uncomfortable: with the fiber lens in place, the detected field seems to be determined mainly by an equivalent field added to stop the incident field at the opaque screen).

In the following, we will be interested in time delays between pairs of scattering events and points in the aperture. From this point on, it will be more convenient to shift our origin of time so that time is measure at the aperture time $t_{a}=t-R_{f} / c$ and write

$$
E\left(P_{o}, t_{a}+\frac{R_{f}}{c}\right)=\frac{-1}{2 \pi} R_{o} \iint_{A} d A \frac{1}{R_{P_{o} A}^{2}}\left\{\frac{1}{R_{P_{o} A}}+2 \frac{1}{c} \frac{\partial}{\partial t}\right\} E\left(A, t_{a}\right)
$$

The time dependent field at the fiber at $t_{a}+R_{f} / C$ is connected with a superposition of fields and their time derivatives that occur simultaneously at the aperture at $t_{a}$. 


\subsubsection{Comment on lack of integration over the fiber end face}

We briefly examine a possible improvement in the approximation where we took the field at the fiber end face that propagates in the fiber as the value at the center of the single mode fiber: $E(P, t) \rightarrow E\left(P_{0}, t\right)$. A single mode fiber propagates a single Gaussian spatial mode. It should be possible to expand the diffraction field $E(P, t)$ in a complete set of orthogonal functions with the symmetry of the fiber, one of which is the fiber's Gaussian-electromagnetic mode. A better approximation for the detected field would be to "couple" the diffraction field calculation to the Gaussian mode by sifting out the amplitude of that function from an expansion in a set of functions. If $G_{f}(P)$ is the fiber mode, then the part of the diffraction field that propagates is proportional to $\int d A_{f} G_{f}(P) E(P, t)$. I.e., instead of taking $E(P, t) \rightarrow E\left(P_{0}, t\right)$ as the detected field, a better approximation would be to integrate over the Gaussian component at the fiber and replace $E(P, t) \rightarrow \int d A_{f} G_{f}(P) E(P, t)$. For points $P$ near and not at $P_{o}$, this introduces an additional phase shift $k \delta(A, P)$ that depends on both aperture and fiber coordinates. By taking the Fourier transform $U_{\omega}(A) \rightarrow E(t)$ at fixed aperture and fiber coordinates, the net effect in the detected field calculation is to replace the field inside the diffraction integral with

$E\left(A, t-R_{f} / c\right) \rightarrow E\left(A, t-\left(R_{f}+\delta(A, P)\right) / c\right)$

Intuition says the net effect should be to cause a spatial oscillation of the field over the fiber end face and alter or suppress coupling at large angles into the fiber. For simulations involving integration over the target, aperture, and fiber, this requires integration in six dimensions. In this report, the author avoids the increase in computation time for an integration over the fiber end face, and instead uses $E(P, t) \rightarrow E\left(P_{0}, t\right)$ for the detected field. This should be an adequate approximation for large experiment aspect ratios. Large angles from large aperture points may be slightly overrepresented in the present simulations.

\subsection{Scalar light-field components scattered from target to aperture in the paraxial approximation}

We shift our attention to deriving the field in the aperture $E\left(A, t_{a}\right)$. The field in the aperture is from the field scattered from the target. We will obtain a result that is exact in the phase. For this paraxial scalar approximation we claim the amplitude is good to about $0.5 \%$. The field at a point in the aperture $\vec{r}_{a}$ is a superposition of scattered fields delayed from points $\vec{r}_{i}$ in the target. We will calculate the field 
component $E_{i a}$ for a pair of points $\vec{r}_{i}, \vec{r}_{a}$, and perform a superposition of the scattered light fields by integrating over the target and aperture.

In order to see easily the form of the scattered light from the moving target, we will shift our frame of reference between the aperture (stationary in the laboratory frame) and the rest frame of our moving target (by frame we mean one moving with constant velocity relative to the other, $\vec{u}_{p}$ ). We will break this problem down into two parts, one for the phases, and one for the amplitudes. In the derivation we will use: invariance for the value of the phase and speed of light observed in different reference frames, the constant value of the phase on a spherical wave surface, and the fact that the amplitude transforms to the same value with additional small terms of $\operatorname{order} \beta$.

An observer in the rest frame of the target uses primed coordinates $\vec{r}^{\prime}, t^{\prime}$, and an observer in the laboratory frame uses un-primed $\vec{r}, t$. Two observers that see the same physical event such as absorption or scattering describe the space time event location with different numbers $\vec{r}^{\prime}=\vec{r}^{\prime}\left(t^{\prime}\right)$ or $\vec{r}=\vec{r}(t)$. A continuous-wave laser field in the laboratory frame is a harmonic function, $E_{\ell} e^{i \varphi_{\ell}}$. In the lab frame, the laser phase $\varphi_{\ell}(\vec{r}, t)$ has a space-independent term $\omega_{\ell} t$ that advances the same way in time for all points in space, i.e., it's spectrum measured anywhere in the lab frame is a delta function at $\omega_{\ell}$. The dependence of $E_{\ell}(\vec{r})$ and $\varphi_{\ell}(\vec{r}, t)$ on spatial coordinates is nonlinear. As viewed from the target, the laser source is moving. The amplitude picks up time dependence $E_{\ell}{ }^{\prime}=E_{\ell}{ }^{\prime}\left(\vec{r}^{\prime}, t^{\prime}\right)$, and the phase $\phi_{\ell}^{\prime}\left(\vec{r}^{\prime}, t^{\prime}\right)$ becomes non-linear in time. The field seen at the target $E_{\ell}^{\prime} e^{i \varphi_{\ell}^{\prime}}$ is no longer strictly harmonic (phase may advance nonlinearly in time), but for small relative velocity $\beta=u_{p} / c$ and slowly varying dependence of $E_{\ell}(\vec{r})$ and $\varphi_{\ell}(\vec{r}, t)$ on $\vec{r}$ we expect the spectrum of $E_{\ell}^{\prime} e^{i \varphi_{\ell}^{\prime}}$ to be narrow. But the laser spectrum is not a harmonic delta function in the target frame.

We consider the form of the scattered wave in the target frame. The laser field $\vec{E}_{\ell}{ }^{\prime} e^{i \varphi^{\prime} \ell}$ applies a force on a charge $q$ with effective mass $m^{\prime}$ resulting in acceleration $\vec{a}^{\prime}=q \vec{E}_{\ell}^{\prime} e^{i \varphi_{\ell}^{\prime}} e^{i \varphi_{0}^{\prime}} / m^{\prime}$. We include a phase shift $e^{i \varphi_{0}^{\prime}}$ that depends on material response - we will consider only homogeneous materials where the phase shift is the same for all scattering events and this will drop out later. In relativistic electrodynamics, an accelerated charge at $\vec{r}_{i}^{\prime}$ has a transverse radiation field that propagates to $\vec{r}^{\prime}$ in the target frame as (see Jackson's chapter 14) as

$$
\vec{E}_{i}^{\prime}=\frac{q}{c}\left[\frac{\hat{R}^{\prime} \times\left(\hat{R}^{\prime} \times \vec{a}^{\prime} / c\right)}{R^{\prime}}\right]_{r e t}=\frac{q^{2}}{m^{\prime} c^{2}}\left[\frac{\hat{R}^{\prime} \times\left(\hat{R}^{\prime} \times \vec{E}_{\ell}^{\prime} e^{i \varphi^{\prime}} e^{i \varphi_{0}^{\prime}}\right)}{R^{\prime}}\right]_{r e t},
$$

where $\vec{R}^{\prime}=\vec{r}^{\prime}-\vec{r}_{i}^{\prime}$ and $R^{\prime}=\left|\vec{r}^{\prime}-\vec{r}_{i}^{\prime}\right|$. The subscript "ret" means the expression for $\vec{E}_{i}{ }^{\prime}$ at $\left(\vec{r}^{\prime}, t\right.$ ') has the same value as the quantity in the square brackets evaluated at the earlier scattering-event time $t^{\prime}-\left|\vec{r}^{\prime}-\vec{r}_{i}^{\prime}\right| / c$. 
For this work, we will use a scalar paraxial approximation for the field amplitude good to about $0.5 \%$ in the amplitude, and exact in the phase. We consider only problems where the positions $\vec{r}_{i}^{\prime}$ and $\vec{r}^{\prime}$ are close to the optical axis and the beam convergence angle is less than 0.1 Radians. The radiation fields (laser and scatter) are nearly transverse to the optic axis. In this paraxial approximation $\hat{R}^{\prime} \times\left(\hat{R}^{\prime} \times \vec{E}_{\ell}{ }^{\prime}\right) \cong-\vec{E}_{\ell}{ }^{\prime}$. In the remainder of this work, we take the scalar amplitudes $E_{\ell}{ }^{\prime}$ and $E_{i}{ }^{\prime}$ as the transverse scalar components of the field $\vec{E}_{\ell}$ ' and ignore small corrections for the amplitude away from the optical axis due to beam divergence-convergence and the dipolar variation of the scattered field with angle. Because we ignore direction cosines in the components of $\vec{E}_{\ell}^{\prime}$, the paraxial approximation introduces an error of order $1-\hat{x} \cdot \hat{R}^{\prime} \stackrel{\sim}{\sim} 0.5 \%$ in the amplitudes. In the paraxial approximation we write the nearly-transverse scalar component of the scattered field $E_{i}$ ' as proportional to the nearlytransverse scalar component of the laser field (and ignore the common overall phase shift):

$$
E_{i}^{\prime}=\frac{q^{2}}{m^{\prime} c^{2}}\left[\frac{E_{\ell} e^{i \phi_{\ell}^{\prime}}}{R^{\prime}}\right]_{\text {ret }} .
$$

(an improvement over this approach beyond the $0.5 \%$ level for the amplitudes would include factors of order unity depending on angles). The phase of $E^{\prime}{ }_{i}$ at time $t^{\prime}$ and distance $R^{\prime}$ is the same as the value $\left[E_{\ell} e^{i \phi_{\ell}^{\prime}}\right]_{\text {ret }}$ at the scattering event at earlier time at $t^{\prime}-\left|\vec{r}^{\prime}-\vec{r}_{i}^{\prime}\right| / c$ (ignoring constant shifts in common). When two observers compare measurements of the same propagating transverse electromagnetic field components $E$ and $B \approx E / C$ in two frames, the field components transform linearly as $E=E^{\prime}-u_{p} B^{\prime} \approx E^{\prime}-\beta E^{\prime} \approx E^{\prime}$. When two observers compare notes, they say also that $\left|\vec{r}-\vec{r}_{i}\right| \approx\left|\vec{r}^{\prime}-\vec{r}_{i}^{\prime}\right| / \gamma, m=\gamma m^{\prime}$ and find that the factors of $\gamma$ roughly cancel for the scattered light amplitude $E_{i}=E_{i}{ }^{\prime}$ (where $\gamma^{2}=1 /\left(1-\beta^{2}\right)$ is nearly equal to 1 ). In other words at low velocities, the two observers see the same field amplitude $E_{i}=E_{i}{ }^{\prime}$ with an error of order $\beta \leq 10^{-4}$, much smaller than our paraxial approximation error of $0.5 \%$.

Phase values are frame invariant (any two observers see the same phase value for the same event), and the two observers agree exactly that $\varphi_{\ell}=\varphi_{\ell}{ }^{\prime}$ and $\varphi_{0}=\varphi_{0}{ }^{\prime}$. Hence, to an approximation exact in phase and to within an error of order $1-\hat{x} \cdot \hat{R}^{\prime} \stackrel{\sim}{<} 0.5 \%$ for the amplitudes, and the complex-number value for the scattered light observed at the detector is

$$
E_{i}=E_{i}^{\prime}=\frac{q^{2}}{m^{\prime} c^{2}} \frac{1}{\left|\vec{r}^{\prime}-\vec{r}_{i}^{\prime}\right|}\left[E_{\ell}^{\prime} e^{i \varphi_{\ell}^{\prime}}\right]_{r e t} e^{i \varphi_{0}^{\prime}}=\frac{q^{2}}{m c^{2}} \frac{1}{\left|\vec{r}-\vec{r}_{i}\right|}\left[E_{\ell} e^{i \varphi_{\ell}}\right]_{r e t} e^{i \varphi_{0}}
$$

At this level of approximation, the observers agree on the functional form and numerical value for the field, but the coordinate values used by the two observers in the expressions are different. For a 
harmonic laser field $E_{\ell} e^{i \varphi_{\ell}}$ in the lab frame, we can write down a functional form for $E_{\ell}=E_{\ell}(\vec{r})$ and $\varphi_{\ell}=\varphi_{\ell}(\vec{r}, t)$ that satisfies the wave equation (we will introduce Gaussian beam expressions in sections 4.3 and 5.0). We can use invariance of $c$ and $\varphi_{\ell}=\varphi_{\ell}{ }^{\prime}$ for the same scattering event observed in two difference reference frames to realize the functional form for the delayed coordinates in the expression $\left[E_{\ell} e^{i \phi_{\ell}}\right]_{\text {ret }}$ in the laboratory-frame coordinates. When observers in the laboratory or target frames examine the scattered light on a spherical surface of radius $c T=\left|\vec{r}-\vec{r}_{i}\right|$ or $c T^{\prime}=\left|\vec{r}^{\prime}-\vec{r}_{i}^{\prime}\right|$ at a time delay $T$ or $T^{\prime}$ after the scattering event, the amplitude is found to have dipole variation in angles, but the phase is found to have the same constant value everywhere on the sphere (which is the same as the phase at the scattering event at the earlier times $t-T$ and $\left.t^{\prime}-T^{\prime}\right)$. In other words, the two observers agree the laser has phase $\varphi_{\ell}\left(\vec{r}_{i}, t-T\right)=\varphi_{\ell}^{\prime}\left(\vec{r}_{i}^{\prime}, t^{\prime}-T^{\prime}\right)$, where $\vec{r}_{i}=\vec{r}_{i}(t-T)$ and $\vec{r}_{i}^{\prime}=\vec{r}_{i}^{\prime}\left(t^{\prime}-T^{\prime}\right)$ are the functions for the positions at the scattering event time. In the target frame, $\vec{r}_{i}^{\prime}=\vec{r}_{i}^{\prime}\left(t^{\prime}-T^{\prime}\right)$ is a constant function. In the lab frame, $\vec{r}_{i}=\vec{r}_{i}(t-T)$ changes in time $t$ because the target is moving with respect to the laboratory. When the sphere of constant phase value equal to $\phi_{\ell}\left(\vec{r}_{i}, t-T\right)$ reaches a point $\vec{r}_{a}$ in the aperture at time $t_{a}$, the scattered light phase $\varphi_{s}$ at the aperture is related to the laser beam phase $\varphi_{\ell}$ at a scattering event that occurred at $\vec{r}_{i}, t_{a}-T_{i a}$, i.e., via $\varphi_{s}\left(\vec{r}_{a}, t_{a}\right)=\varphi_{\ell}\left(\vec{r}_{i}, t_{a}-T_{i a}\right)$. In other words the scattered light originating at $\vec{r}_{i}=\vec{r}_{i}\left(t_{a}-T_{i a}\right)$ has scalar field value at the aperture with a functional dependence on laboratory coordinates as $E_{i a}=\frac{q^{2}}{m c^{2}} \frac{1}{\left|\vec{r}_{a}-\vec{r}_{i}\right|} E_{\ell}\left(\vec{r}_{i}\right) e^{i \phi_{\ell}\left(\vec{r}_{i}, t_{a}-T_{i a}\right)} e^{i \phi_{0}}$

Due to phase invariance in the two frames and the constancy of the phase on the light sphere, the phase $\varphi_{s}\left(\vec{r}_{a}, t_{a}\right)=\varphi_{\ell}\left(\vec{r}_{i}, t_{a}-T_{i a}\right)$ is exact. Due to the paraxial approximation for the transverse scalar field, we may take the amplitude $E_{\ell}=E_{\ell}\left(\vec{r}_{i}\right)$ to within an error $1-\hat{x} \cdot \hat{R}^{\prime} \stackrel{\sim}{\sim} 0.5 \%$, with the argument for the laser field $\vec{r}_{i}$ now changing in time as the function $\vec{r}_{i}=\vec{r}_{i}\left(t_{a}-T_{i a}\right)$. This time dependence of the position argument in the field amplitude arises due to the apparent motion of the laser source in the target frame, and is part of the reason the scattered light field is no longer harmonic.

Because the target is moving in the lab frame, the optical time delay between scattering event and aperture position is also a function of positions and time, $T_{i a}=T_{i a}\left(\vec{r}_{i}, \vec{r}_{a}, t_{a}\right)$. In other words, we have two relations with $\vec{r}_{i}=\vec{r}_{i}\left(t_{a}-T_{i a}\right)$ that depends on $T_{i a}$, and $T_{i a}=T_{i a}\left(\vec{r}_{i}, \vec{r}_{a}, t_{a}\right)$ that depends on $\vec{r}_{i}$. In order to write a closed form expression for $E_{i a}$, we need to relate $\vec{r}_{i}=\vec{r}_{i}\left(t_{a}-T_{i a}\right)$ and $T_{i a}=T_{i a}\left(\vec{r}_{i}, \vec{r}_{a}, t_{a}\right)$ to an experiment configuration, solve for $T_{i a}$ and $\vec{r}_{i}$ separately in terms of experiment parameters (e.g., in terms of laser field function, initial positions $\vec{r}_{i 0}$, a uniform velocity $\vec{u}_{p}$, and lens delay $\delta=\delta\left(\vec{r}_{a}\right)$ ). 
With a set of initial positions $\vec{r}_{i 0}$ traveling at constant, uniform mass velocity $\vec{u}_{p}$ with positions $\vec{r}=\vec{r}_{i 0}+t \vec{u}_{p}$, the positions of scattering events were $\vec{r}_{i}=\vec{r}_{i}\left(t_{a}-T_{i a}\right)=\vec{r}_{i 0}+\left(t-T_{i a}\right) \vec{u}_{p}$. With no target lens in the aperture, $T_{i a}$ is the time delay $T_{i a}=\left|\vec{r}_{a}-\vec{r}_{i}\right| / c$ for a spherical wave. Our infinitesimally thin lenses do not take up physical space, but the target lens does have an effective thickness $\delta$. The actual delay from scattering event to aperture has an additional delay due to the target lens, and so the relation for $T_{i a}$ including the additional delay in the lens is $c T_{i a}=\left|\vec{r}_{a}-\vec{r}_{i}\right|+\delta$. Now, we to select a form for the lens effective thickness, $\delta=\delta\left(\vec{r}_{a}\right)$, and solve for $T_{i a}$ and $\vec{r}_{i}$.

\subsection{Closed-form solutions for propagation time $T_{i a}$ and its derivative $\dot{T}_{i a}$}

\section{including target-lens delay $\delta\left(\vec{r}_{a}\right) / C$}

For the fiber lens, we had used a trick to define the lens delay that would give a constant time delay from aperture points to the fiber. The reader may amuse himself by trying to create a perfect lens with constant phase delay between all object and image points in all of space for all of time and for all wavelengths. The contradictions reached during this exercise may be taken as proof that there are no perfect lenses that can image all space-time points without aberration. (This was not a problem for a single point at the end of the fiber).

For the target lens we need to make a choice for the function $\delta=\delta\left(\vec{r}_{a}\right)$ in order to perform simulations. We take a practical approach and chose a polynomial dependence that will create an image, albeit imperfect. Following Yariv and Goodman, an adequate polynomial for a paraxial system is a simple quadratic in the radius $r_{a}=\left|\vec{r}_{a}\right|$ of an aperture point. A phase delay of the form $k \delta\left(\vec{r}_{a}\right)=k\left(\delta_{0}-r_{a}^{2} / 2 f\right)$ focuses a plane wave at focal distance $f$. For our work we choose a nonzero value $\delta_{0}$ on the axis so that $\delta=\delta\left(\vec{r}_{a}\right)$ goes to zero at the aperture edges for a particular focal length $f$.

For a particular experiment, there will be a set of initial positions $\vec{r}_{i 0}$ for the target and particle velocity $\vec{u}_{p}$ from which we may write down functions $\vec{r}_{i}=\vec{r}_{i}\left(t_{a}-T_{i a}\right)$. In our present problem solution for an opaque reflective surface travelling uniformly with velocity $\vec{u}_{p}$, scattering events from one $\vec{r}_{i 0}$ occur along a straight line $\vec{r}_{i}=\vec{r}_{i 0}+\left(t_{a}-T_{i a}\right) \vec{u}_{p}$ with events occurring at the earlier time $t_{a}-T_{i a}$. Due to the simplicity of the relation $\vec{r}_{i}=\vec{r}_{i}\left(t_{a}-T_{i a}\right)$ for a constant $\vec{u}_{p}$, we are able to solve the pair of relations $\vec{r}_{i}=\vec{r}_{i 0}+\left(t_{a}-T_{i a}\right) \vec{u}_{p}$ and $c T_{i a}=\left|\vec{r}_{a}-\vec{r}_{i}\right|+\delta\left(\vec{r}_{a}\right)$ algebraically at time $t$ to find $T_{i a}$ in terms of constant values $\vec{r}_{i 0}, \vec{r}_{a}, \vec{u}_{p}$ and the delay $\delta=\delta\left(\vec{r}_{a}\right)$. The solution for $T_{i a}$ is the non-negative value of 


$$
\begin{aligned}
& T_{i a}=t_{a}-\gamma^{2}\left[\left(t_{a}-\frac{\delta\left(\vec{r}_{a}\right)}{c}\right)+\left(\vec{\beta} \cdot \frac{\Delta \vec{r}_{0}}{c}\right)\right] \\
& \pm \gamma^{2}\left[\beta^{2}\left(t_{a}-\frac{\delta\left(\vec{r}_{a}\right)}{c}\right)^{2}+\left(1-\beta^{2}\right) \frac{\Delta \vec{r}_{0}}{c} \cdot \frac{\Delta \vec{r}_{0}}{c}+\left(\vec{\beta} \cdot \frac{\Delta \vec{r}_{0}}{c}\right)^{2}+2\left(t_{a}-\frac{\delta\left(\vec{r}_{a}\right)}{c}\right)\left(\vec{\beta} \cdot \frac{\Delta \vec{r}_{0}}{c}\right)\right]^{1 / 2}
\end{aligned}
$$

Here, $\Delta \vec{r}_{0}=\vec{r}_{i 0}-\vec{r}_{a}$ is the difference between target initial position and the aperture position, $\vec{\beta}=\vec{u}_{p} / c$, and $\gamma^{2}=1 /\left(1-\beta^{2}\right)$. This closed form expression for $T_{i a}$ is useful in numerical calculations where we consider the scattering events to occur along $\vec{r}_{i}=\vec{r}_{i 0}+\left(t_{a}-T_{i a}\right) \vec{u}_{p}$ with an amplitude for the events that we will connect with the laser amplitude. The reader may find it simpler to set up calculations with $\vec{r}_{i 0}$ pulled back to a "place before the experiment starts" and avoid letting the problem evolve through the initial positions or through the aperture (one may need only a single choice for the \pm sign in that case). The reader may verify that in a very rough sense, $T_{i a}$ becomes more nonlinear for scattering events "close to the aperture" and becomes more nearly linear for scattering events "far from the aperture." For motion far and away from the aperture, the delay time back to the aperture $T_{i a}$ asymptotes to a fixed fraction:

$T_{i a} \rightarrow \frac{\beta}{(1+\beta)} t_{a}$

We will see later (e.g. section 4.3 for motion in a Gaussian laser field) that an expression for the rate of change $\partial T_{i a} / \partial t$ is needed for an understanding of the Doppler shift. Instead of taking the derivative of the solution for $T_{i a}$, one arrives to a compact form with a simple description more quickly if one starts instead by taking the time derivative of $c T_{i a}=\left|\vec{r}_{a}-\vec{r}_{i}\right|+\delta\left(\vec{r}_{a}\right)$ :

$$
\frac{\partial T_{i a}}{\partial t}=\left(1-\frac{\partial T_{i a}}{\partial t}\right) \vec{\beta} \cdot \frac{\vec{r}_{i}-\vec{r}_{a}}{\left|\vec{r}_{i}-\vec{r}_{a}\right|},
$$

or since $\beta<<1$,

$$
\frac{\partial T_{i a}}{\partial t}=\vec{\beta} \cdot \frac{\vec{r}_{i}-\vec{r}_{a}}{\left|\vec{r}_{i}-\vec{r}_{a}\right|} .
$$

The rate of change with time of the delay $T_{i a}$ from scattering position to aperture is a component of the relative velocity $\vec{\beta}=\vec{u}_{p} / c$ along the line joining a scattering point to an aperture position. 


\subsection{The complete superposition of fields scattered from target points $\vec{r}_{i}$}

\section{that arrive simultaneously at aperture points $r_{a}$}

Having found that there are circumstances were we can write down a closed form solution for $T_{i a}=T_{i a}\left(\vec{r}_{i}, \vec{r}_{a}, t_{a}\right)$ we return to writing the total scattered field amplitude at an aperture point in terms of $\vec{r}_{i}=\vec{r}_{i 0}+\left(t_{a}-T_{i a}\right) \vec{u}_{p}, \varphi_{s}\left(\vec{r}_{a}, t_{a}\right)=\varphi_{\ell}\left(\vec{r}_{i}, t_{a}-T_{i a}\right)$, and $E_{\ell}=E_{\ell}\left(\vec{r}_{i}\right)$. We consider a superposition of scattered fields that contribute to the total field at an aperture point $\vec{r}_{a}$ at some aperture time $t_{a}$. The different delays $T_{i a}$ depend on the aperture time, velocity, initial positions, and aperture point, $T_{i a}=T_{i a}\left(\vec{u}_{p}, \vec{r}_{i 0}, \vec{r}_{a}, t_{a}\right)$. Due to the nonlinear behavior of $T_{i a}$, the surface of scattering events $\vec{r}_{i}=\vec{r}_{i 0}+\left(t_{a}-T_{i a}\right) \vec{u}_{p}$ is a slightly curved surface for a set of initial positions $\vec{r}_{i 0}$ arranged in a plane. In order to correctly integrate the density of scattering points to create a superposition at $\vec{r}_{a}$ we may imagine an integral over the surface $\vec{r}_{i}$ with curvilinear coordinates $Y, Z$ in the surface. $Y, Z$ coordinates are not convenient to use but inspire instead the following. We introduce an orthogonal Cartesian coordinate frame for the laboratory with origin in the aperture, $\hat{x}$ along the fiber axis (confocal optical axis), and $\hat{y}$ and $\hat{z}$ in the aperture plane. Another set of integration variables to consider are components of the initial positions $y_{i 0}, z_{i 0}$ (expressed in aperture plane coordinates). For a planar target surface, we can relate $x_{i 0}=x_{i 0}\left(y_{i 0}, z_{i 0}\right)$ expressed in aperture coordinates. The expression for $x_{i 0}=x_{i 0}\left(y_{i 0}, z_{i 0}\right)$ can be written in terms of the surface normal $\hat{n}$ for the target surface. The difference between a pair of points in the initial position plane is perpendicular to $\hat{n}$. For example,

$\hat{n} \cdot\left(\vec{r}_{i 0}-x_{00} \hat{x}\right)=n_{x}\left(x_{i 0}{ }^{\prime \prime}-x_{00}{ }^{\prime \prime}\right)+n_{y} y_{i 0}{ }^{\prime \prime}+n_{z} z_{i 0}{ }^{\prime \prime}=0$

where $x_{00} \hat{x}$ is the initial position on the beam axis. We have calculated the Jacobian relating $d Y d Z$ to $d y_{i 0} d z_{i 0}$. For low velocities, the Jacobian factors connecting $d Y d Z$ with $d y_{i 0} d z_{i 0}$ are of order $1 / n_{x}$ with extra terms of order $\partial T_{i a} / y_{i 0} \approx \beta<<1$. (one realizes $n_{x}$ is the familiar cosine projection of area $d Y d Z$ into area $\left.d y_{i 0} d z_{i 0}\right)$. Because the extra terms are of order $\beta \leq 10^{-4}$, we ignore the very small curvature introduced by $T_{i a}=T_{i a}\left(\vec{u}_{p}, \vec{r}_{i 0}, \vec{r}_{a}, t_{a}\right)$ into the surface $\vec{r}_{i}$ at low velocities and replace the summation over the surface coordinates $Y, Z$ with a summation over initial positions $y_{i 0}, Z_{i 0}$ with $d Y d Z=d y_{i 0} d z_{i 0} / n_{x}$. 
To project the scattered field amplitude into the plane of aperture $A$, we introduce formally an "obliquity" factor $O_{i a}$. Since polarization of the light in PDV is usually not controlled and unknown due to birefringence in the fiber, we will not have much to say about $O_{i a}$ in this report other than it is related to the projection of the polarization of the light and is of order unity for nearly paraxial conditions.

Collecting factors, we arrive finally at the superposition of scattered fields at an aperture point $\vec{r}_{a}$ parameterized by the initial positions $\vec{r}_{i 0}$

$E_{s}\left(\vec{r}_{a}, t_{a}\right)=\frac{q^{2}}{m c^{2}} e^{i \phi_{0}} \iint d y_{i 0} d z_{i 0} \frac{1}{n_{x}} \frac{O_{i a}}{R_{i a}} E_{\ell}\left(\vec{r}_{i}\right) e^{i \phi_{\ell}\left(\vec{r}_{i}, t_{a}-T_{i a}\right)}$.

For calculating the instantaneous signal sideband peak frequency $\omega_{p}$ we will take a ratio that will cancel constant factors and at various stages we keep only the proportional parts. Keep in mind that while we are integrating over initial positions $\vec{r}_{i 0}$, there is an implied "at the time $t_{a}$ " in the integral since $\vec{r}_{i}=\vec{r}_{i 0}+\left(t_{a}-T_{i a}\right) \vec{u}_{p}$ and $T_{i a}=T_{i a}\left(\vec{u}_{p}, \vec{r}_{i 0}, \vec{r}_{a}, t_{a}\right)$, and this expression takes into account all amplitudes of scattered fields at different times $t_{a}-T_{i a}$ and positions $\vec{r}_{i}$ that arrive at the point $\vec{r}_{a}, t_{a}$. Substituting $E_{s}\left(\vec{r}_{a}, t_{a}\right)$ into the expression relating the field at the aperture to the field at the point $P_{0}$ (the fiber end face), we arrive at a superposition of fields proportional to the detected field.

$$
\begin{aligned}
E_{s}\left(P_{o}, t_{a}+\frac{R_{f}}{c}\right) & =\frac{-1}{2 \pi} R_{o} \iint_{A} d y_{a} d z_{a} \frac{1}{R_{P_{o} A}^{2}}\left\{\frac{1}{R_{P_{o} A}}+2 \frac{1}{c} \frac{\partial}{\partial t}\right\} \iint d y_{i 0} d z_{i 0} \frac{1}{n_{x}} \frac{O_{i a}}{R_{i a}} E_{\ell}\left(\vec{r}_{i}\right) e^{i \phi_{\ell}\left(\vec{r}_{i}, t_{a}-T_{i a}\right)} \\
& =\frac{-1}{2 \pi} R_{o} \frac{1}{n_{x}} \iint_{A} d y_{a} d z_{a} \frac{1}{R_{P_{o} A}^{2}} \iint d y_{i 0} d z_{i 0}\left\{\frac{1}{R_{P_{o} A}}+2 \frac{1}{c} \frac{\partial}{\partial t}\right\} \frac{O_{i a}}{R_{i a}} E_{\ell}\left(\vec{r}_{i}\right) e^{i \phi_{\ell}\left(\vec{r}_{i}, t_{a}-T_{i a}\right)}
\end{aligned}
$$

This expression involves a summation over points $\vec{r}_{i 0}$ in the target surface and summation over points $\vec{r}_{a}$ in the aperture. Although this is not a true path integral formulation, one may think of this as a summation of amplitudes over all paths starting from all points $\vec{r}_{i}$ in the target surface passing through all points $\vec{r}_{a}$ in the lens aperture and arriving at the fiber end face point $P_{0}$. The time derivative that appears in this expression was introduced from the diffraction integral calculation and remains in this expression since the scattered light fields from a moving surface are not harmonic.

\subsection{Reversing the order of integration:}

A geometric description of the superposition of scattered fields from one target point $\vec{r}_{i 0}$ 
Figure 3.6.1 describes an alternate geometric description of the integration process with the order of integration reversed. For each initial position in the target, integration is performed first over the lens aperture.

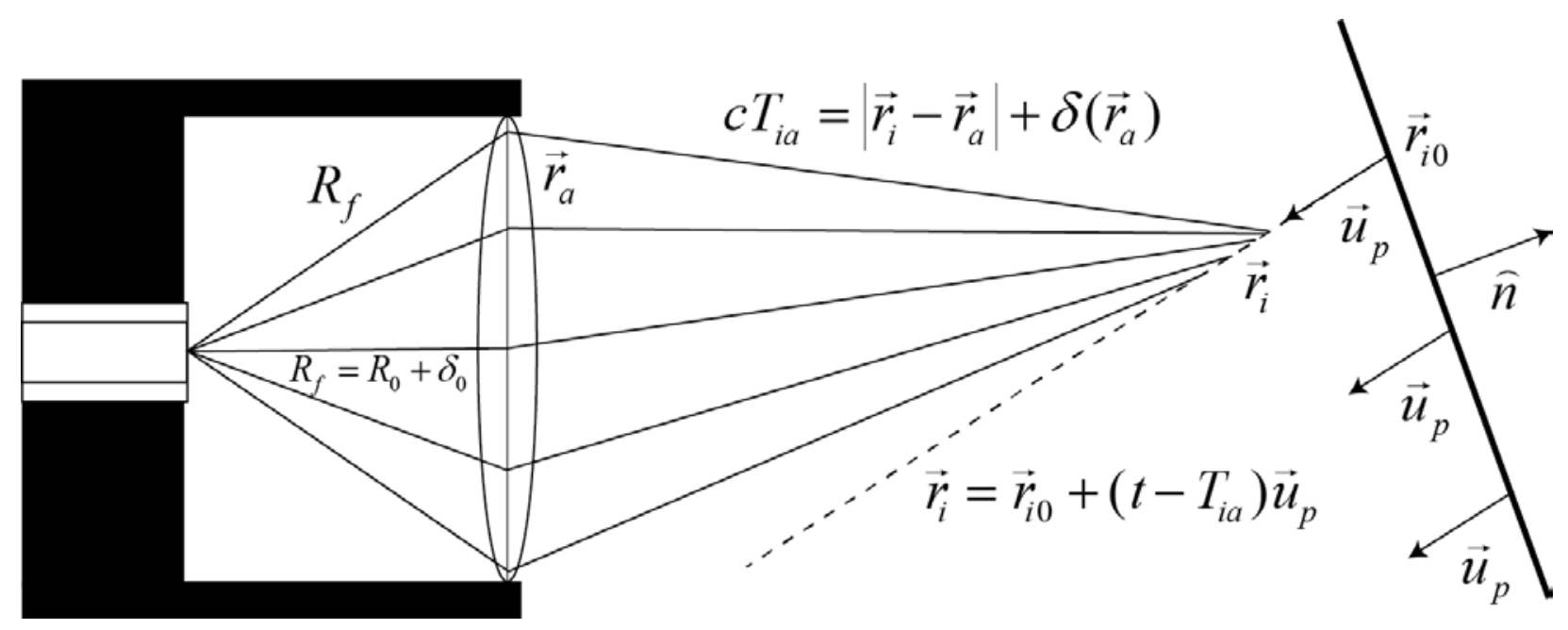

Figure 3.6.1. Geometrical description of scattered light superposition integration from the point of view of one initial position in the target. Dimensions are exaggerated.

A target consists of initial positions $\vec{r}_{i 0}$ at $t=0$. In this report, target points have uniform and constant particle velocity $\vec{u}_{p}$ for all $t$. The delay through the left lens is defined so all paths between aperture and fiber have the same delay time $R_{f} / C$. Scattered light-field components superposed at the fiber at time $t+R_{f} / c$ had arrived simultaneously at the aperture at time $t$. A particular point labelled $\vec{r}_{i 0}$ determines a straight line path of scattered light positions $\vec{r}_{i}=\vec{r}_{i 0}+\left(t-T_{i a}\right) \vec{u}_{p}$, that occur at earlier times $t-T_{i a}$. The propagation delay $T_{i a}$ between scattering point $\vec{r}_{i}$ and lens aperture point $\vec{r}_{a}$ includes a right-lens delay $\delta\left(\vec{r}_{a}\right) / c$, i.e., $c T_{i a}=\left|\vec{r}_{i}-\vec{r}_{a}\right|+\delta\left(\vec{r}_{a}\right)$. For particular values of $t, \vec{r}_{i 0}, \vec{u}_{p}$, and $\vec{r}_{a}$, the two relations $\vec{r}_{i}=\vec{r}_{i}\left(\vec{r}_{i 0}, \vec{u}_{p}, t, T_{i a}\right)$ and $T_{i a}=T_{i a}\left(\vec{r}_{a}, \delta\left(\vec{r}_{a}\right), \vec{r}_{i}\right)$ determine the corresponding $T_{i a}$ and $\vec{r}_{i}$, which become arguments to the scattered light field component value at the aperture $O_{i a} E_{\ell}\left(\vec{r}_{i}\right) e^{i \phi_{\ell}\left(\vec{r}_{i}, t_{a}-T_{i a}\right)} / R_{i a}$. Before integrating over initial positions, one may integrate all light from one target point over the lens aperture. From this perspective, we realize that the scattered light observed at $t$ from one target point is not scattered from a unique position or time, but may be smeared out in space and time to a small extent. This space-time aberration for a real lens and moving target may not be discernible for low enough velocities, but is a real effect included in our calculations. Integration over all aperture points $\vec{r}_{a}$ and all initial points $\vec{r}_{i 0}$ completes the superposition of scattered light components at the fiber. 
CHAPTER 4. Expressing $\omega_{p}$ in terms of the diffraction result for $E_{r}^{*} E_{s}$

\subsection{Instantaneous PDV-signal frequency expressed in terms of the time dependent scattered fields}

We are interested in the instantaneous peak frequency $\omega_{p}$ for the sideband spectral peak in the detected power which requires forming the product $E_{r}^{*} E_{s}$ and a ratio of $\partial\left(E_{r}^{*} E_{s}\right) / \partial t$ and $E_{r}{ }^{*} E_{s}$ (section 2.1). To complete a calculation for $\omega_{p}$ we will need a specific functional form for the laser field. In the usual confocal optical arrangement, the laser light emerges from a single mode optical fiber and propagates to the target as a Gaussian beam function (Yariv section 2.5). In the remainder of this development, we will use a Gaussian beam function for the laser and write this with a subscript " $\mathrm{g}$ " as $E_{\ell}\left(\vec{r}_{i}\right) e^{i \varphi_{\ell}\left(\vec{r}_{i}, t_{a}-T_{i a}\right)}=E_{g}\left(\vec{r}_{i}\right) e^{i \varphi_{g}\left(\vec{r}_{i}, t_{a}-T_{i a}\right)}$.

For the signal product $E_{r}^{*} E_{s}$, we will absorb the harmonic factor $E_{r}{ }^{*}$ inside the integral for $E_{s}$ as follows. Scattering a harmonic laser field by a moving target introduces a non-harmonic time dependent term $-\omega_{r}\left(t_{a}-T_{i a}\right)$ into the scattered light phase $\varphi_{g}\left(\vec{r}_{i}, t_{a}-T_{i a}\right)$. In a moment, we will use a result arising from taking a derivative of a product of $E_{r}^{*}$ and the integrand:

$$
\frac{\partial}{\partial t}\left(E_{r}^{*} \frac{O_{i a}}{R_{i a}} E_{g}\left(\vec{r}_{i}\right) e^{i \varphi_{g}\left(\vec{r}_{i}, t_{a}-T_{i a}\right)}\right)=\left|E_{r}\right| \frac{\partial}{\partial t}\left(e^{+i \omega_{r} t_{a}} \frac{O_{i a}}{R_{i a}} E_{g}\left(\vec{r}_{i}\right) e^{i \varphi_{g}\left(\vec{r}_{i}, t_{a}-T_{i a}\right)}\right)
$$

The harmonic time dependence $\omega_{r} t_{a}$ in the reference field $E_{r}{ }^{*}$ combines with the term $-\omega_{r}\left(t_{a}-T_{i a}\right)$ in the laser phase $\varphi_{g}\left(\vec{r}_{i}, t_{a}-T_{i a}\right)$ and vastly reduces the time advance to a lower rate $\omega_{r} t_{a}-\omega_{r}\left(t_{a}-T_{i a}\right)=\omega_{r} T_{i a}$ (the phase difference of two optical fields cancels in the signal cross term leaving a phase that advances at microwave frequencies). As a shorthand, the phase difference between the reference and the scattered light phase can be expressed by replacing the time argument in the laser phase function with $t_{a}-T_{i a} \rightarrow-T_{i a}$, i.e., $\omega_{r} t_{a}+\varphi_{g}\left(\vec{r}_{i}, t_{a}-T_{i a}\right)=\varphi_{g}\left(\vec{r}_{i},-T_{i a}\right)$. After absorbing the reference field phase $\omega_{r} t_{a}$ we write

$$
\frac{\partial}{\partial t}\left(E_{r}^{*} \frac{O_{i a}}{R_{i a}} E_{g}\left(\vec{r}_{i}\right) e^{i \varphi_{g}\left(\vec{r}_{i}, t_{a}-T_{i a}\right)}\right)=\left|E_{r}\right| \frac{\partial}{\partial t}\left(\frac{O_{i a}}{R_{i a}} E_{g}\left(\vec{r}_{i}\right) e^{i \varphi_{g}\left(\vec{r}_{i},-T_{i a}\right)}\right)
$$


Expanding and rearranging we find a term that appears when we bring $E_{r}^{*}$ inside the integral expressed using $\phi_{g}\left(\vec{r}_{i},-T_{i a}\right)$ :

$$
\left|E_{r}\right| e^{+i \omega_{r} t_{a}} \frac{\partial}{\partial t}\left\{\frac{O_{i a}}{R_{i a}} E_{g}\left(\vec{r}_{i}\right) e^{i \varphi_{g}\left(\vec{r}_{i}, t_{a}-T_{i a}\right)}\right\}=\left|E_{r}\right| \frac{\partial}{\partial t}\left\{\frac{O_{i a}}{R_{i a}} E_{g}\left(\vec{r}_{i}\right) e^{i \varphi_{g}\left(\vec{r}_{i},-T_{i a}\right)}\right\}-\left|E_{r}\right| i \omega_{r}\left\{\frac{O_{i a}}{R_{i a}} E_{g}\left(\vec{r}_{i}\right) e^{i \varphi_{g}\left(\vec{r}_{i},-T_{i a}\right)}\right\}
$$

Using these expressions, the product $E_{r}{ }^{*} E_{s}$ becomes (to within a constant phase factor from $E_{r}{ }^{*}$ that divides out later)

$$
E_{r}^{*} E_{s}=\left|E_{r}\right| \frac{-1}{2 \pi} R_{0} \frac{1}{n_{x}} \iint_{A} d y_{a} d z_{a} \frac{1}{R_{P_{0} A}^{2}} \iint d y_{i 0} d z_{i 0}\left\{\frac{1}{R_{P_{0} A}}-2 i \frac{\omega_{r}}{c}+2 \frac{1}{c} \frac{\partial}{\partial t_{a}}\right\}\left(\frac{O_{i a}}{R_{i a}} E_{g}\left(\vec{r}_{i}\right) e^{i \varphi_{g}\left(\vec{r}_{i},-T_{i a}\right)}\right)
$$

We pause again to trace the origin of terms. The term $1 / R_{P_{0} A}$ in curly braces arose from the straight summation of fields at the aperture in the diffraction calculation. The remaining terms $(2 / c)\left(-i \omega_{r}+\partial / \partial t_{a}\right)$ arose from the time derivative in the diffraction integral needed due to the presences of the absorbing aperture and lens. As written, all the time dependent parts, $R_{i a}=\left|\vec{r}_{i}-\vec{r}_{a}\right|$, $E_{g}\left(\vec{r}_{i}\right), \varphi_{g}\left(\vec{r}_{i},-T_{i a}\right), T_{i a}=T_{i a}\left(\vec{u}_{p}, \vec{r}_{i 0}, \vec{r}_{a}, t_{a}\right)$, and $\vec{r}_{i}=\vec{r}_{i 0}+\left(t_{a}-T_{i a}\right) \vec{u}_{p}$ are confined to the right side of the integrand after the operator in curly brackets. To finish the calculation of $\omega_{p}$, it is now straight forward to move the derivative $\partial\left(E_{r}^{*} E_{s}\right) / \partial t$ in the numerator for $\omega_{p}$ inside the integral past the curly braces:

$$
\frac{\partial}{\partial t_{a}} \ldots\left\{\frac{1}{R_{P_{0} A}}-2 i \frac{\omega_{r}}{c}+2 \frac{1}{c} \frac{\partial}{\partial t_{a}}\right\} \rightarrow\left\{\frac{1}{R_{P_{0} A}}-2 i \frac{\omega_{r}}{c}+2 \frac{1}{c} \frac{\partial}{\partial t_{a}}\right\} \frac{\partial}{\partial t_{a}}
$$

The time derivative on the right is connected with the derivative for the phase difference needed in the calculation for $\omega_{p}$. The terms inside the curly brackets are from the diffraction calculation. We now have all the computational elements needed for simulating and calculating the instantaneous frequency $\omega_{p}$.

We consider several related questions: Why does the diffraction calculation lead to a complicated expression for the signal frequency; what are the relative sizes of terms; when are any of the terms considerably smaller than the others (and may be ignored); and what is the meaning of the dominant terms in the expression for $\omega_{p}$ ?

We wish to examine the relative sizes of the terms resulting from the curly brackets $\{. .$.$\} . To keep track$ of the results, we will refer to the terms as 
1: $\frac{1}{R_{P_{0} A}}$

2: $-2 i \frac{\omega_{r}}{c}$

3: $: 2 \frac{1}{c} \frac{\partial}{\partial t_{a}}$

1 and 2]: In this paper we will work with a focusing probe similar to those used in gas gun targets. Focusing probes used in PDV are usually magnifying-focuser probes, have a lens aperture radius less than $4 \mathrm{~mm}$ and typically $\sim 1 \mathrm{~mm}$, and a working distance between the aperture to the focal position in the laser beam that is $10 \mathrm{~mm}$ or more. Within the curly brackets $\{\ldots\}$, the factor $2 \omega_{r} / c$ is also $2 \omega_{r} / c=2 k=4 \pi / \lambda$. PDV typically operates with $\lambda=1.55 \times 10^{-6} \mathrm{~m}$. Written in units of $\lambda$, the distance between fiber and lens is more than 1000 wavelengths, i.e., $R_{P_{0} A} \sim 10^{3} \lambda$. The factor $2 \omega_{r} / C$ is larger than the term $1 / R_{P_{0} A}$ by more than a factor of $10^{4}$ for practical values of $R_{P_{0} A}$.

2 and 3: Next, we consider the third term. The derivative in the curly brackets $\{\ldots\}$ can be expanded in two terms $3 A$ and $3 B$ :

$$
2 \frac{1}{c} \frac{\partial}{\partial t_{a}}\left[\frac{O_{i a}}{R_{i a}} E_{g}\left(\vec{r}_{i}\right) e^{i \phi_{g}\left(\vec{r}_{i},-T_{i a}\right)}\right]=2 \frac{1}{c}\left[\left(\frac{R_{i a}}{O_{i a} E_{g}\left(\vec{r}_{i}\right)}\right) \frac{\partial}{\partial t_{a}}\left(\frac{O_{i a} E_{g}\left(\vec{r}_{i}\right)}{R_{i a}}\right)-i \omega_{i a}\right]\left[\frac{O_{i a}}{R_{i a}} E_{g}\left(\vec{r}_{i}\right) e^{i \phi_{g}\left(\vec{r}_{i},-T_{i a}\right)}\right]
$$

Here we define the derivative of the phase factor as a frequency

3B: $\omega_{i a} \equiv-\partial \phi_{g}\left(\vec{r}_{i},-T_{i a}\right) / \partial t$

labelled with scattering point and aperture point. This frequency is the time rate of change of the difference in phase between the reference field and a scattered light field for the pair of points $\vec{r}_{i}$ to $\vec{r}_{a}$. (Under somewhat idealized circumstances where the first term disappears, one may think of $\omega_{i a}$ loosely as a Doppler shift contribution to a single spectrum for this pair of points). We will see (section 4.3) that the contributions $\omega_{i a}$ are nearly equal to $2 \omega_{r} \beta_{x}=2 \omega_{r} \beta \cos (\theta)$ where the subscript $x$ means $\beta_{x}=u_{p x} / c$ is related to the component of the motion along the beam direction, and $\theta$ is the angle of the particle velocity with respect to the optical axis (i.e. is connected with the Doppler shift). $3 A$ : The other term in the first square [...] bracket is the fractional rate of change of $O_{i a} E_{g}\left(\vec{r}_{i}\right) / R_{i a}$ caused by motion $\vec{r}_{i}=\vec{r}_{i 0}+\left(t_{a}-T_{i a}\right) \vec{u}_{p}$ of scatterers in the beam. Generally we expect a multiplicative amplitude modulation factor to have a spectrum that convolves symmetrically with the signal spectrum and does 
not affect the peak frequency. $3 A$ is a kind of additive, motion-induced amplitude modulation term that does affect the signal spectrum. We will restrict our present discussion to places in the laser beam not close to the lens (i.e., $R_{i a}$ is not small). Intuitively the largest rate of change of $O_{i a} E_{g}\left(\vec{r}_{i}\right) / R_{i a}$ will occur when the direction of motion is highly transverse to a narrow part of the beam, i.e., is largest when crossing the laser focus transversely. Roughly, we expect the fractional rate of amplitude variation $3 A$ is of order no larger than $\left(u_{p} \sin (\theta) / W_{0}\right)$. In most practical situations the laser beam radius at the focus, $W_{0}$, is more than $10 \lambda$. Multiplying the two terms not larger than $3 A: u_{p} / W_{0}$ and $3 B: 2 \omega_{r} \beta$ by the prefactor $2 / c$, we expect that the magnitude of terms arising from the derivative in the curly brackets $\{\ldots\}$ is less than $(4 \pi / \lambda) 2 \beta$. For most terrestrial problems with $\beta \stackrel{\sim}{<} 10^{-4}$, the middle term 2$]:\left|-2 i \omega_{r} / c\right|=2 k=4 \pi / \lambda$ will be $10^{4}$ larger than the 3 . Hence, for most problems involving practical focusing probes, we can safely ignore all terms compared to $-2 i \omega_{r} / c$, and approximate the curly brackets using

$$
\left\{\frac{1}{R_{P_{0} A}}-2 i \frac{\omega_{r}}{c}+2 \frac{1}{c} \frac{\partial}{\partial t_{a}}\right\} \cdot \rightarrow\left\{-2 i \omega_{r} / c\right\}
$$

To an approximation with relative error not larger than $10^{-4}$, we can approximate $E_{r}{ }^{*} E_{s}$ by

$$
E_{r}^{*} E_{s}=\left\{-2 i \frac{\omega_{r}}{c}\right\}\left|E_{r}\right| \frac{-1}{2 \pi} R_{0} \frac{1}{n_{x}} \iint_{A} d y_{a} d z_{a} \frac{1}{R_{P_{0} A}^{2}} \iint d y_{i 0} d z_{i 0} \frac{O_{i a}}{R_{i a}} E_{g}\left(\vec{r}_{i}\right) e^{i \varphi_{g}\left(\vec{r}_{i},-T_{i a}\right)} .
$$

When we trace the origin of terms backwards to the diffraction calculation, we learn that the integrand factor $O_{i a} E_{g}\left(\vec{r}_{i}\right) e^{i \phi_{g}\left(\vec{r}_{i},-T_{i a}\right)} / R_{i a}$ in the expression for the cross term $E_{r}^{*} E_{s}$ has lineage back to the time derivative term in the diffraction calculation. The dominant term arose from the wavelength dependence of the gradient of the Green's function through the aperture, and is connected with an "extra" field generated by the aperture edges in the absorbing screen. The terms related to the straight summation of fields in the aperture in the diffraction calculation were negligible compared to the time derivative term. Strangely, it appears that the motion of charges in the edges of the aperture needed to cancel the field there contribute a "source" to the propagating field, which is more important than if the aperture were not there. After multiplying through by the harmonic function $E_{r}^{*}$ the phase in the cross term $E_{r}^{*} E_{s}$ slows down from the phase advance in either $E_{r}^{*}$ or $E_{s}$ and the time derivative of the field $E_{s}$ from the aperture-edge field resolves into a dominant term with prefactor $\left\{-2 i \omega_{r} / c\right\}$ (and no time derivative). 
Now we consider the expression in the numerator of $\omega_{p}$ with time derivative. Similarly, when computing $\partial\left(E_{r}^{*} E_{s}\right) / \partial t$ in the calculation of $\omega_{p}$, we can bring the derivative inside the integral, use similar arguments about the relative sizes of terms and arrive at the same conclusion that the dominant term in integrand in the numerator becomes

$\left\{\frac{1}{R_{P_{0} A}}-2 i \frac{\omega_{r}}{c}+2 \frac{1}{c} \frac{\partial}{\partial t_{a}}\right\} \frac{\partial}{\partial t_{a}} \rightarrow\left\{-2 i \frac{\omega_{r}}{c}\right\} \frac{\partial}{\partial t_{a}}$

When computing the ratio in $\omega_{p}$ many constant factors divide out. When including only the dominant term $10^{4}$ larger than other terms, $\omega_{p}$ becomes

$$
\begin{aligned}
\omega_{p}=-\operatorname{Im}\left[\frac{1}{E_{r}^{*} E_{s}} \frac{\partial}{\partial t} E_{r}^{*} E_{s}\right] \\
\left.=-\operatorname{Im}\left[\frac{\iint_{A} d y_{a} d z_{a} \frac{1}{R_{P_{0} A}^{2}} \iint d y_{i 0} d z_{i 0} \frac{\partial}{\partial t}\left[\frac{O_{i a}}{R_{i a}} E_{g}\left(\vec{r}_{i}\right) e^{i \phi_{g}\left(\vec{r}_{i},-T_{i a}\right)}\right]}{\iint_{A} d y_{a} d z_{a} \frac{1}{R_{P_{0} A}^{2}} \iint d y_{i 0} d z_{i 0}\left[\frac{O_{i a}}{R_{i a}} E_{g}\left(\vec{r}_{i}\right) e^{i \phi_{g}\left(\vec{r}_{i},-T_{i a}\right)}\right]}\right]\right]
\end{aligned}
$$

The time derivative in the numerator of the last expression is traceable to the operation $\omega_{p}=\partial \Phi / \partial t$ needed to convert the phase difference $\Phi$ of the integral for $E_{r}^{*} E_{s}$ to a frequency. This is the complete expression we will use in simulations of the measured velocity in PDV.

\subsection{What happens to the expression for $\omega_{p}$ for small angles?}

A few experimental tests have been performed in an attempt to reveal "what PDV measures" under asymmetric conditions such as particle velocity that is not along the optical axis [Briggs and Dolan]. Many of these experimental tests were performed while the surface was tilted also. From the available information at small angles one may conclude that the PDV frequency is proportional to the component of the particle velocity along the optical axis. We now examine how this arises in the previous expression for $\omega_{p}$.

Writing out the derivative we obtain an expression in terms of the individual Doppler contributions $\omega_{i a}=-\partial \varphi_{g}\left(\vec{r}_{i},-T_{i a}\right) / \partial t$ and an amplitude modulation-like term related to the fractional rate of 
change due to transit through the laser beam:

$$
\omega_{p}=-\operatorname{Im}\left[\frac{\iint_{A} d y_{a} d z_{a} \frac{1}{R_{P_{0} A}^{2}} \iint d y_{i 0} d z_{i 0}\left[\left(\frac{R_{i a}}{O_{i a} E_{g}\left(\vec{r}_{i}\right)}\right) \frac{\partial}{\partial t_{a}}\left(\frac{O_{i a} E_{g}\left(\vec{r}_{i}\right)}{R_{i a}}\right)-i \omega_{i a}\right]\left[\frac{O_{i a}}{R_{i a}} E_{g}\left(\vec{r}_{i}\right) e^{i \phi_{g}\left(\vec{r}_{i},-T_{i a}\right)}\right]}{\iint_{A} d y_{a} d z_{a} \frac{1}{R_{P_{0} A}^{2}} \iint d y_{i 0} d z_{i 0}\left[\frac{O_{i a}}{R_{i a}} E_{g}\left(\vec{r}_{i}\right) e^{i \phi_{g}\left(\vec{r}_{i},-T_{i a}\right)}\right]}\right]
$$

The frequency contributions $\omega_{i a}$ appear in the numerator after performing the operation related to compute $\omega_{p}=\partial \Phi / \partial t$ in $E_{r}^{*} E_{s}$. Similarly to section 4.1, we will label the two terms in the numerator

$\underline{3 A}:\left(\frac{R_{i a}}{O_{i a} E_{g}\left(\vec{r}_{i}\right)}\right) \frac{\partial}{\partial t_{a}}\left(\frac{O_{i a} E_{g}\left(\vec{r}_{i}\right)}{R_{i a}}\right)$

$3 B: \omega_{i a}=-\partial \phi_{g}\left(\vec{r}_{i},-T_{i a}\right) / \partial t$

First, consider a "one dimensional" experiment with smooth planar surface with surface normal and particle velocity parallel to the optical axis $\hat{n} / / \vec{u}_{p} / / \hat{x}$, i.e., $\theta=0$. Consider a perpendicular radius $r_{\perp}$ where $r_{\perp}{ }^{2}=y_{i}{ }^{2}+z_{i}{ }^{2} . O_{i a} E_{g} / R_{i a}$ is peaked at $r_{\perp}=0$, which means derivatives of $O_{i a} E_{g} / R_{i a}$ are odd functions under the replacement of integration variables $y_{i 0} \rightarrow-y_{i 0}, z_{i 0} \rightarrow-z_{i 0}$. So for completely symmetric one dimensional conditions, the integral over the first term in the square brackets sums to zero, and $\omega_{p}$ simplifies to

$\left.\omega_{p}=\operatorname{Re}\left[\frac{\left\{\iint_{A} d y_{a} d z_{a} \frac{1}{R_{P_{0} A}^{2}} \iint d y_{i 0} d z_{i 0}\left[\frac{O_{i a}}{R_{i a}} E_{g}\left(\vec{r}_{i}\right) e^{i \phi_{g}\left(\vec{r}_{i},-T_{i a}\right)}\right] \omega_{i a}\right\}}{\left\{\iint_{A} d y_{a} d z_{a} \frac{1}{R_{P_{0} A}^{2}} \iint d y_{i 0} d z_{i 0}\left[\frac{O_{i a}}{R_{i a}} E_{g}\left(\vec{r}_{i}\right) e^{i \phi_{g}\left(\vec{r}_{i},-T_{i a}\right)}\right]\right.}\right\}\right]$

(Here we used $\operatorname{Im}[i()]=\operatorname{Re}[()]$ ). Intuitively, we expect that the term $3 A$ that was zero for $\theta=0$ will remain small for some range of small $\theta$ values:

$\left|\left(\frac{R_{i a}}{O_{i a} E_{g}\left(\vec{r}_{i}\right)}\right) \frac{\partial}{\partial t_{a}}\left(\frac{O_{i a} E_{g}\left(\vec{r}_{i}\right)}{R_{i a}}\right)\right|<<\left|\omega_{i a}\right|$, for small angles.

We will examine whether and where this may breakdown in section 4.4. 
We see that for small angles $\omega_{p}$ is closely related to a weighted average of frequencies $\omega_{i a}$ with weighting by complex field amplitudes (with cancellations and additions that are reminiscent of coherent interference). A similar expression was obtained for a complete expansion in harmonic functions in section 2.2 However, we did not arrive at this expression with time domain functions completely expanded in harmonic functions. We kept the time dependent Gaussian beam phase that satisfies the time dependent wave equation. This introduces a hypothesis that seems reasonable from an intuitive experimental perspective: the scattered light field in a nearly one dimensional experiment is nearly harmonic, and the spread in the spectrum of scattered fields may be narrow (but not zero), especially under symmetric circumstances. We examine the spread in the spectrum $\omega_{i a}$ for small angles in the next section 4.3.

\subsection{Values of $\omega_{i a}$ when scattering points are moving in a Gaussian laser field}

and scatter light into lens aperture points

We compute $\omega_{i a}=-\partial \varphi_{g}\left(\vec{r}_{i},-T_{i a}\right) / \partial t$ to see it's narrow spectrum of values for a "real" harmonic laser field that satisfies the wave equation. For a confocal arrangement with light sent to the target on single mode fiber and using lenses that are not too small, the laser field near the target is a "Gaussian Mode" (Yariv section 2.5). The "beam" has a finite lateral extent with field amplitude decreasing with distance perpendicular to the laser beam $r_{\perp}^{2}=y^{2}+z^{2}$ as a Gaussian function $(W o / W) \operatorname{EXP}\left(-\left(r_{\perp} / W\right)^{2}\right)$.

The beam radius converges and diverges as

$W=W_{o} \sqrt{1+\left(x_{i}-f\right)^{2} / X_{R}^{2}}$

with a minimum radius $W=W_{o}$ at the focal position $x_{i}=f$. The beam radius increases by a factor

$\sqrt{2}$ at the distance $x_{i}-f$ from focus equal to the Rayleigh range parameter $X_{R}=\pi W_{0}^{2} / \lambda$. A Gaussian beam is completely determined by a minimum radius $W=W_{o}$ and the wavelength $\lambda$.

For a Gaussian laser beam with axis along the $\hat{X}$ direction and minimum radius located at the focus at $x=f$, the phase of the laser field depends on spatial coordinates and time as

$\phi_{g}(\vec{r}, t)=k(x-f)-\tan ^{-1}\left(\frac{x-f}{X_{R}}\right)+\frac{1}{2} \frac{r_{\perp}{ }^{2} k(x-f)}{(x-f)^{2}+X_{R}{ }^{2}}-\omega_{r} t$. 
The first and last terms $k(x-f)$ and $-\omega_{r} t$ resemble the two terms for a plane wave $\phi(\vec{r}, t)=\vec{k} \bullet \vec{r}-\omega t$, with wavevector $\vec{k}=k \hat{x}$ pointing along the optical axis in the $\hat{x}$ direction. A Gaussian beam converges and diverges through it's minimum radius $W_{o}$ with phase-front curvature described by the term $(1 / 2) r_{\perp}{ }^{2} k(x-f) /\left[(x-f)^{2}+X_{R}{ }^{2}\right]$. Also due to beam convergence and divergence, the phase acquires an additional slowly varying term $-\tan ^{-1}\left((x "-f) / X_{R}\right)$ called the Guoy phase that various between $\pm \pi / 2$ over a distance comparable to $X_{R}$.

For the calculation of the PDV signal frequency, we need the difference in phase between the scattered light phase and the reference phase. Due to the motion of the target (or rather the motion of the laser source relative to the target), the phase difference between scattered light and the reference phase, $\varphi_{g}\left(\vec{r}_{i},-T_{i a}\right)$, is non-linear in time due to the replacements $-\omega_{r} t \rightarrow+\omega_{r} T_{i a}$ and $\vec{r}_{i}=\vec{r}_{i 0}+\left(t_{a}-T_{i a}\right) \vec{u}_{p}$, and due to the optical time delay being non-linear in time. Taking the derivative, we obtain the frequency contribution for scattered light from a Gaussian laser beam to points in a lens aperture as

$$
\begin{aligned}
-\omega_{i a}= & \left.\frac{\partial \phi_{g}}{\partial t}\right|_{t=t_{a}} \\
= & {\left[\begin{array}{l}
1-\frac{X_{R}{ }^{2}}{X_{R}{ }^{2}+\left(x_{i}-f\right)^{2}} \frac{1}{k X_{R}} \\
\left.1-\frac{\partial}{\partial t} T_{i a}\right)\left[\begin{array}{l}
+\frac{1}{\left(x_{i}-f\right)^{2}+X_{R}{ }^{2}}\left(x_{i}-f\right)\left(\frac{\beta_{y}}{\beta_{x}} y_{i}+\frac{\beta_{z}}{\beta_{x}} z_{i}\right) \\
+\frac{1}{2} \frac{X_{R}{ }^{2}-\left(x_{i}-f\right)^{2}}{\left[\left(x_{i}-f\right)^{2}+X_{R}{ }^{2}\right]^{2}}\left(y_{i}{ }^{2}+z_{i}{ }^{2}\right)
\end{array}\right]+\left.\omega_{r} \frac{\partial}{\partial t} T_{i a}\right|_{t_{a}}
\end{array}\right] }
\end{aligned}
$$

We use a subscript $x$ on the factor $\omega_{r} \beta_{x}$ to mean the component of $\beta_{x}=u_{p x} / c$ along the optical axis (which happens to be the same direction as the "laser beam" for confocal optics).

We examine the relative contributions of terms in $\omega_{i a}$. We will show that there are two terms nearly equal to $\omega_{r} \beta_{x}$, all other terms can be much smaller, and $\omega_{i a}$ is nearly equal to $2 \omega_{r} \beta_{x}$. The derivative of the first three space-like terms in $\varphi_{g}$ (i.e., not the $\omega_{r} T_{i a}$ term) introduce a common factor $\omega_{r} \beta_{x}\left(1-\partial T_{i a} / \partial t\right)$. Because $\partial T_{i a} / \partial t=\vec{\beta} \cdot\left(\vec{r}_{i}-\vec{r}_{a}\right) /\left|\vec{r}_{i}-\vec{r}_{a}\right|$ is of order $\beta \widetilde{<} 10^{-4}$, we will ignore the extra term of order $\beta<<1$ in the factor $\left(1-\partial T_{i a} / \partial t\right) \approx(1-\beta) \approx 1$. In effect, we can ignore the dependence on aperture position in the factors $\left(1-\partial T_{i a} / \partial t\right)$ on the space-like phase terms. Contributions to $\omega_{i a}$ from the three space-like terms depend mainly on the position within the laser beam and depend very little on the points in the aperture. 
For the moment, let's ignore the curvature and imagine we have only a plane wave term $\vec{k} \bullet \vec{r}$. The frequency shift contribution from the space-like term becomes

$$
\frac{\partial}{\partial t} \vec{k} \cdot \vec{r}_{i}=\vec{k} \cdot \vec{u}_{p}\left(1-\frac{\partial}{\partial t} T_{i a}\right)=\omega_{r} \hat{k} \cdot \vec{\beta}\left(1-\frac{\partial}{\partial t} T_{i a}\right) \approx \omega_{r} \hat{k} \cdot \vec{\beta} \text {, because } \frac{\partial}{\partial t} T_{i a} \approx \beta<<1
$$

Notice in this last expression that the rate of change of the plane wave part of the phase does not depend on the initial positions of the scatterers or the location of the lens, of course. And this contribution to the frequency shift depends on the component of the velocity $\vec{\beta}$ along the direction $\hat{k}$ for the plane wave part of the beam phase. Now, for a plane wave (or beam) along the lens axis $(\hat{x})$, the first dominant term is simply $\omega_{r} \beta_{x}$. One of the two dominant terms comes from the space-like planewave-like term, with the value determined by the fact that PDV typically is confocal.

The second dominant term comes from the rate of change of the optical delay-time between scattering point and the lens aperture. For lens and beam diameters smaller than the separation $\left|\vec{r}_{i}-\vec{r}_{a}\right|$, the difference $\left(\vec{r}_{i}-\vec{r}_{a}\right)$ has a small angle to the optical axis $\hat{x}$, and $\omega_{r} \frac{\partial T_{i a}}{\partial t}=\omega_{r} \vec{\beta} \cdot \frac{\left(\vec{r}_{i}-\vec{r}_{a}\right)}{\left|\vec{r}_{i}-\vec{r}_{a}\right|} \approx \omega_{r} \beta_{x}$

This frequency from the optical-delay term depends both on the position within the laser beam and on the points within the lens aperture. These two dominant terms from the plane-wave like contributions add nearly to the value $2 \omega_{r} \beta_{x}$ (but not precisely due to the slightly different path lengths for different paths through the lens.

The reader may wish to verify that wave-front curvature contributes terms much smaller than $2 \omega_{r} \beta_{x}$ for various combinations of the conditions: distances not too close to the lens; small transverse beam and lens diameters compared to the distance from the lens; focus diameter that is not too small, direction of motion that is not too transverse to the laser beam. For positions in a beam with small transverse dimension $W$ and motion that is not strongly transverse to the beam direction, i.e., $y_{i}^{2}+z_{i}^{2} \stackrel{\sim}{<} W^{2}<<x_{i}^{2} \approx f^{2}$, and $\beta_{y}<<\beta$, and $\beta_{z}<<\beta$, and $\beta_{x} \approx \beta$, the terms in $\omega_{i a}$ from converging-beam effects (Guoy phase and curvature terms) tend to be smaller than $2 \omega_{r} \beta_{x}$ by factors of $1 / k X_{R}$ or $\left(W / X_{R}\right)^{2}$. When moving along the beam and near the beam focus, the additional space-like term contributions do grow but start out very small and of order $\sim 10^{-4}$, even for a tightly focused beam with minimum beam width $(W / \lambda) \sim 20$. The curvature effects depend on the Gaussian beam parameters (minimum radius $W_{o}$ and wavelength $\lambda$ ) and do not depend on the lens aperture diameter. 
Since the optical time delay depends on a scattering position within the beam and an aperture point, more significant deviations from $2 \omega_{r} \beta_{x}$ are found from the optical delay due to the factor

$\left(\vec{r}_{i}-\vec{r}_{a}\right) /\left|\vec{r}_{i}-\vec{r}_{a}\right|$, even for motion $\vec{\beta}$ directed along the optical axis. For $\left|\vec{r}_{i}-\vec{r}_{a}\right| \approx 10 \mathrm{~mm}$, lens radius of order $2 \mathrm{~mm}$, and beam width much narrower than the lens, variation of $\omega_{i a}$ away from the value $2 \omega_{r} \beta_{x}$ can be as large as $(2 / 10)^{2} / 4 \approx 1 \%$ near the edges of the lens (perhaps disappointingly, a large lens used for high light collection efficiency introduces a small spread in the frequency spectrum). For a highly symmetric situation with no roughness, there tends to be oscillations of contributions with cancellations of $\omega_{i a}$ values out near the edges of the lens, and the total deviations as large as $1 \%$ do not occur for a smooth symmetric experiment. For nearly symmetric or nearly one-dimensional situations, we find a result consistent with experimental observations that PDV measures a value similar to $2 \omega_{r} \beta_{x}$ to better than $1 \%$ (in the first simulation of Chapter 5 , we find this result to better than $0.05 \%$ at 5 degrees for a smooth surface, but this begins to break down as roughness increases).

\subsection{What happens to the value of $\omega_{p}$ at large angles?}

Detailed cancellations may be disrupted for less symmetric situations. For situations that are less symmetric due to $\hat{n}, \vec{u}_{p}$, and $\hat{x}$ not being parallel, there is at least one additional term $3 A$ involving a fractional rate of change of $O_{i a} E_{g}\left(\vec{r}_{i}\right) / R_{i a}$ that we cannot ignore. We may think of this term as a coupling of frequency and amplitude modulation due to motion in the beam (there are other types of amplitude motion that may not have this coupling to the frequency). One of the reasons that this term arises is that the scattered waves from individual points on a moving target from a moving laser are not harmonic functions, i.e., have a spectrum. In the development of expressions for $\omega_{p}$ we started with a spectrum of harmonic functions, used the Kirchhoff integral relation to propagate harmonic functions from the aperture and lens to the fiber, but then transformed the expressions over to the time domain to relate this to the non-harmonic time dependent scattered fields at the lens aperture. Hence, the expression for $\omega_{p}$ is not just related to $\omega_{i a}$, but acquires additional time dependent terms for the motion of the laser beam relative to the target. When the symmetry is lowered, e.g. by surface roughness, and the phase shifts are not symmetric for replacement of integral variables $y_{i 0} \rightarrow-y_{i 0}, z_{i 0} \rightarrow-z_{i 0}$, the cancellations across the beam and lens are not guaranteed and larger deviations in frequency occasionally are possible (see Chapter 5).

We would like to obtain an approximate angle where the value of $\omega_{p}$ may begin to deviate from $2 \omega_{r} \beta_{x}$. We showed above that the term $3 B: \omega_{i a} \approx 2 \omega_{r} \beta \cos (\theta)$. We expect intuitively that contributions from the amplitude modulation term are of order $3 A: u_{p} \sin (\theta) / W$. For angle $\theta$ between the optic axis and direction of motion exceeding $\tan (\theta)=4 \pi /(\lambda W)$, i.e., for angles that are larger than 
approximately 45 degrees, we expect that the motion-induced amplitude modulation term may become comparable to the usual "Doppler" term. PDV can be expected to return $\omega_{i a} \approx 2 \omega_{r} \beta \cos (\theta)$ for angles less than 45 degrees, but for larger angles it is not yet clear what will happen (additional simulations beyond those in Chapter 5 are recommended).

In this chapter, we combined the instantaneous frequency $\omega_{p}$ for a heterodyne experiment with classical electromagnetic diffraction field theory and point-wise scattering from a moving target and found this theory consistent with the experimental result, $\omega_{p}=2 \omega_{r} \beta_{x}$ for angles that are not huge. The theory provides the additional insight that the integral for $\omega_{p}$ involves a delicate balancing of contributions over a narrow, complex-value weighted distribution of frequencies.

\section{CHAPTER 5. Simulation results}

\subsection{Simulation parameters}

In this section we describe simulation results for tilted mass velocity and tilted surface with and without roughness. Cartesian coordinates are centered on the optical axis in the lens aperture, with $\hat{x}$ along the optical axis (Figure. 3.2.1) and orthogonal coordinate basis vectors $\hat{y}$ and $\hat{z}$ in the plane of the aperture. The target surface was flat and the particle velocity was uniform and constant. Parameters in Table 5.1 were fixed in common for all simulations in this section.

As we saw in the previous section, we do not expect the amplitude modulation by target motion to affect the apparent velocity results in a perfectly symmetric situation. In high velocity experiments, one cannot always ensure that the configuration is precisely symmetric. In some experiments where tilt may be larger than the numerical aperture of the lens and fiber, we may intentionally add roughness to the surface. We performed simulations for an asymmetric condition with the particle velocity tilted away from the optical axis by 5 degrees $\left(u_{p}=-1000 \mathrm{~m} / \mathrm{s}, u_{p x}=-996 \mathrm{~m} / \mathrm{s}, u_{p y}=-87 \mathrm{~m} / \mathrm{s}\right.$, and $\left.u_{p z}=0\right)$. From a practical perspective, one may imagine also that a probe was not manufactured perfectly, the probe optical axis and probe cylinder centerline are not parallel (fiber or lens off axis), and the optical axis is actually tilted away from the surface normal despite a prefect mechanical assembly external to the probe. For these simulations, we tilted the surface normal 5 degrees $\left(n_{x}=-0.996\right.$, $n_{y}=0$, and $n_{z}=0.087$ ), in a direction different from the particle velocity tilt. These choices were somewhat arbitrary but intended to break the symmetry of the simulation (I encourage the reader to try other choices). The results in this section represent approximately 120 hours of computer simulation. The simulations were performed on a laptop computer with single core processor.

\begin{tabular}{|l|l|l|}
\hline \multicolumn{2}{|c|}{ Table 5.1. Simulation parameters that were fixed for all simulations } \\
\hline & & Comment \\
\hline Probe fiber lens focal length & $R_{o}=3 \mathrm{~mm}$ & \\
\hline
\end{tabular}




\begin{tabular}{|c|c|c|}
\hline Target fiber lens focal length & $f=10 \mathrm{~mm}$ & \\
\hline Lens radius & $1 \mathrm{~mm}$ & \\
\hline Beam waist radius & $W_{o}=15 \mu m$ & \\
\hline Up Particle velocity & $\left|u_{p}\right|=-1000 \mathrm{~m} / \mathrm{s}$ & \\
\hline Upx/Up & $\cos (\theta)=\cos \left(\pi 5^{\circ} / 180^{\circ}\right)$ & \\
\hline Upy/Up & $\sin (\theta)=\sin \left(\pi 5^{\circ} / 180^{\circ}\right)$ & $\begin{array}{l}\text { Particle velocity towards lens and tipped } \\
\text { away from } \mathrm{x} \text { in the } \mathrm{y} \text { direction } 5 \text { degrees }\end{array}$ \\
\hline Upz/Up & 0 & \\
\hline $\mathrm{nx}$ & $\cos (\theta)=\cos \left(\pi 5^{\circ} / 180^{\circ}\right)$ & $\begin{array}{l}\text { Surface normal tipped away from } \mathrm{x} \text { in } \\
\text { the } z \text { direction by } 5 \text { degrees }\end{array}$ \\
\hline ny & 0 & \\
\hline $\mathrm{nz}$ & $\sin (\theta)=\sin \left(\pi 5^{\circ} / 180^{\circ}\right)$ & \\
\hline Laser wavelength $\lambda$ & 1550.3D-9 meters & \\
\hline Integration step size & $1.5 \lambda$ & \\
\hline Number of time points & 71 & \\
\hline Starting position & $10 \mathrm{~mm}$ & \\
\hline End position & $9 \mathrm{~mm}$ & \\
\hline $\begin{array}{l}\text { Initial position of surface at } \\
\text { beam centerline }\end{array}$ & $10.2 \mathrm{~mm}$ & \\
\hline Obliquity factor & $O_{i a}=1$ & \\
\hline
\end{tabular}

The excitation laser field is a focused Gaussian beam (see Yariv's section 2.5, and section 4.3 in this report) along the optical axis $\hat{x}$. The field amplitude decreases away from beam centerline and from the focus as

$E_{g}\left(\vec{r}_{i}\right)=E_{0}\left(W_{0} / W\right) \operatorname{EXP}\left(-\left(r_{i \perp}^{2} / W^{2}\right)\right)$

where $r_{i \perp}{ }^{2}=y_{i}^{2}+z_{i}^{2}$. The beam waist radius was selected to be $W_{o}=15 \mu m$ at the beam focus. The target-lens focal distance and the beam focus coincide for the simulation at a distance $f=10 \mathrm{~mm}$ from the aperture. The beam waist radius $W$ increases from the minimum $W_{o}$ with distance $x_{i}-f$ from the focus as

$W=W_{o} \sqrt{1+\left(x_{i}-f\right)^{2} / X_{R}^{2}}$

The Rayleigh range parameter $X_{R}$ for the laser beam,

$$
X_{R}=\pi W_{o}^{2} / \lambda=\pi(15 \mu m)^{2} /(1.55 \mu m)=455 \mu m,
$$

describes the beam convergence and wave-front curvature. $X_{R}$ is the distance beyond the focus where the beam radius increases by a factor of $\sqrt{2}$, and the power density on the centerline is half the value 
at focus. The laser field is determined by setting the waist radius ( $W_{o}=15 \mu \mathrm{m}$ ) and wavelength ( $\lambda=1.55 \mu \mathrm{m})$.

For this work, the obliquity factor describing the coupling of the scattered light to the aperture or fiber is set to $O_{i a}=1$ (this introduces a field amplitude errors on the order of the squares of direction cosines, or about $0.5 \%)$.

The target moves towards the lens (with mass velocity tilted off axis by 5 degrees, and surface normal tilted in another direction). Each simulations starts with the target surface intersecting the beam centerline close to $10 \mathrm{~mm}$ and moves from $10 \mathrm{~mm}$ to $9 \mathrm{~mm}$ while calculating values of the signal amplitude $E_{r}^{*} E_{s}$ and signal frequency $\omega_{p}$ at 71 time steps.

At each time in the simulations, the instantaneous sideband peak frequency $\omega_{p}$ was calculated using

$$
\begin{aligned}
& \omega_{p}=-\operatorname{Im} {\left[\frac{1}{E_{r}^{*} E_{s}} \frac{\partial}{\partial t} E_{r}^{*} E_{s}\right] } \\
&=-\operatorname{Im}\left[\frac{\iint_{A} d y_{a} d z_{a} \frac{1}{R_{P_{0} A}^{2}} \iint d y_{i 0} d z_{i 0} \frac{\partial}{\partial t}\left[\frac{O_{i a}}{R_{i a}} E_{g}\left(\vec{r}_{i}\right) e^{i \varphi_{g}\left(\vec{r}_{i},-T_{i a}\right)}\right]}{\iint_{A} d y_{a} d z_{a} \frac{1}{R_{P_{0} A}^{2}} \iint d y_{i 0} d z_{i 0}\left[\frac{O_{i a}}{R_{i a}} E_{g}\left(\vec{r}_{i}\right) e^{i \varphi_{g}\left(\vec{r}_{i},-T_{i a}\right)}\right]}\right]
\end{aligned}
$$

(see section 4.1 for this level of approximation). We introduced the functional form for the laser phase $e^{i \phi_{g}\left(\vec{r}_{i},-T_{i a}\right)}$ in an earlier section 4.3 and will not repeat that discussion here. The target plane function $x_{i}=x_{i}\left(y_{i}, z_{i}\right)$ was defined in a previous section 4.3 and depends on the target surface normal $\hat{n}$ via $\hat{n} \cdot\left(\vec{r}_{i 0}-x_{00} \hat{x}\right)=n_{x}\left(x_{i 0}{ }^{\prime \prime}-x_{00}{ }^{\prime}\right)+n_{y} y_{i 0}{ }^{\prime \prime}+n_{z} z_{i 0} "=0$.

The beam waist size at the target grows as the target is moved away from the focus. In this simulation the beam waist radius grows from 15 micrometers to 36 micrometers for target positions from $10 \mathrm{~mm}$ (focus) to $9 \mathrm{~mm}$ from the lens. For these simulations we set the integration subarray size for initial positions to span 132 by 132 micrometers for each time step. The initial position array size (-66 to +66 micrometers in $y_{i 0}$ and $z_{i 0}$ ) and circular-lens radius $(1 \mathrm{~mm})$ are more than 9 times smaller than the distance to the target (with target distances between $10 \mathrm{~mm}$ to $9 \mathrm{~mm}$ ). For each integral at each time step, the phase $\varphi_{g}\left(\vec{r}_{i},-T_{i a}\right)$ varies slowly with changes in lateral positions $y_{i 0}, z_{i 0}, y_{a}$ and $z_{a}$. For integration step sizes equal to $1.5 \lambda$ (in each of the parameters $y_{i 0}, z_{i 0}, y_{a}$ and $z_{a}$ ), the phase value $\varphi_{g}\left(\vec{r}_{i},-T_{i a}\right)$ changes much less than $\pi$ for $y_{i 0}, z_{i 0}, y_{a}$ and $z_{a}$ close to the optical axis (where the beam power density is large) and changes approximately $0.2 \pi$ near the edges of integration sub arrays (where the beam power density is small). 


\subsection{Results with no surface roughness}

PDV is expected to return a value for the signal frequency near $\omega_{p} \simeq 2 \omega_{r} \beta_{x}$. $\beta_{x}$ is the component $u_{p x} / c=u_{p} \cos (\theta) / c$ along the optical axis ( $\theta$ is the angle between the optical axis and the particle velocity.) When $\omega_{p}=2 \omega_{r} \beta_{x}$, we expect the ratio $\omega_{p} / 2 \omega_{r} \beta_{x}$ to be 1 . Small deviations from $\omega_{p}=2 \omega_{r} \beta_{x}$ show up in $\omega_{p} / 2 \omega_{r} \beta_{x}$ as small deviations from 1 . In the following we are interested in these small deviations and plot a percent change $100\left(\omega_{p} / 2 \omega_{r} \beta_{x}-1\right)$.

Figure 5.1 shows an example of results from a simulated PDV experiment with 71 time steps between 10 $\mathrm{mm}$ to $9 \mathrm{~mm}$ with a particle velocity of $u_{p}=-1000 \mathrm{~m} / \mathrm{s}$, tilted five degrees from the optical axis. For this simulation, the surface was smooth (i.e., no roughness was added to $x_{i 0}=x_{i 0}\left(y_{i 0}, z_{i 0}\right)$ ). For this smooth surface in Fig. 5.1, the simulated particle velocity was the same as the component $u_{p x}=-996 \mathrm{~m} / \mathrm{s}$ along the optical axis to better than $0.05 \%$.

For the signal amplitude, we plot the normalized magnitude of the cross term $\left|E_{r}^{*} E_{s}\right|$ in the detected power (this is the integral expression in the denominator for $\omega_{p}$ ). For different values of tilt or when adding roughness, the signal amplitude can vary by a large amount. For display purposes, we normalize the results for the signal amplitude $\left|E_{r}{ }^{*} E_{s}\right|$ to the maximum signal amplitude within the simulation. The power density along the beam centerline varies as $1 /\left[1+\left(x_{i}-f\right)^{2} / X_{R}{ }^{2}\right]$. We expect also the image of the target spot at the fiber to "go out of focus" as the target moves. As expected, as the target moves from the focal position at $10 \mathrm{~mm}$ towards the lens, the signal starts out at a maximum value, and then decreases with a shape similar to a smooth Lorentzian function with distance. 


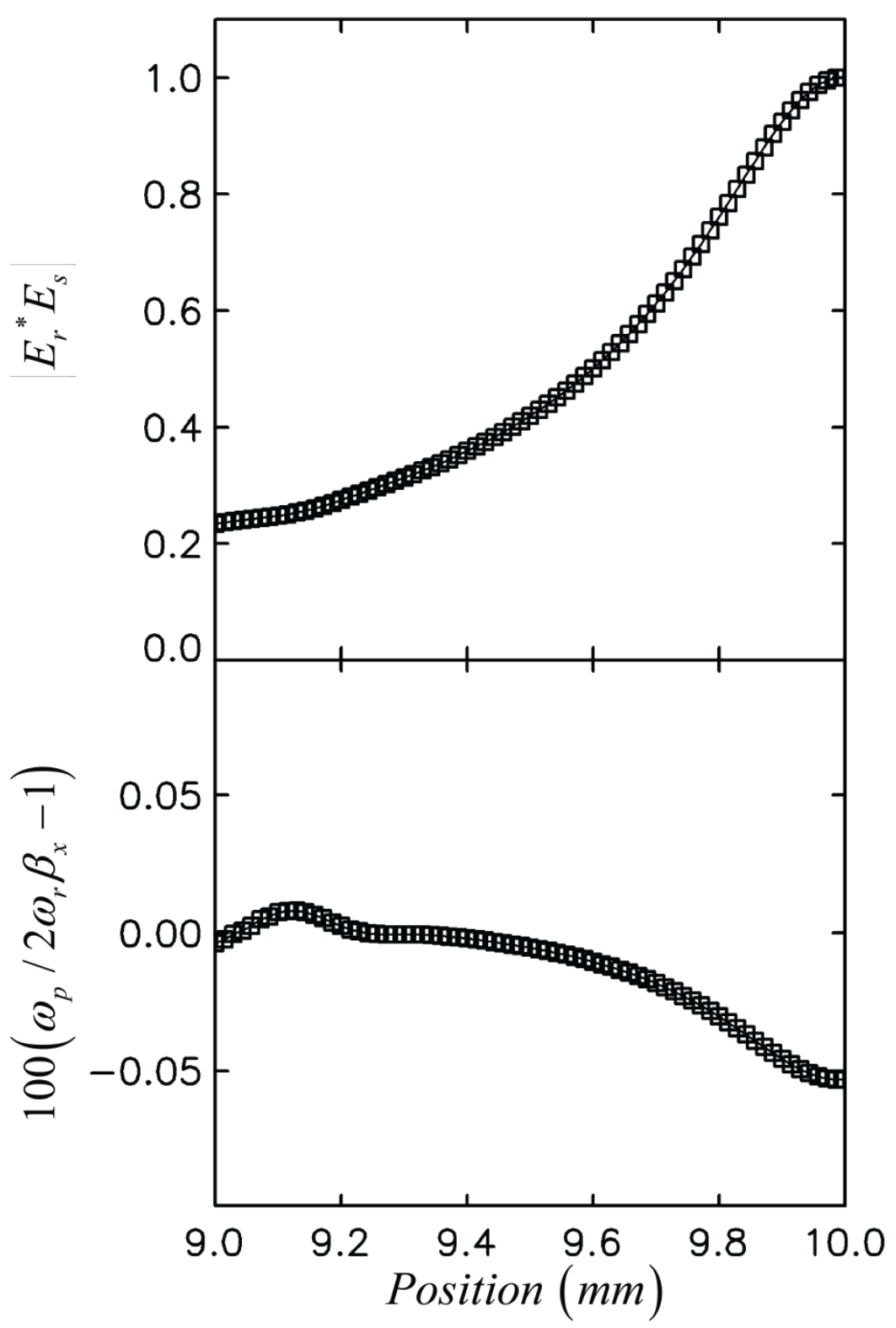

Figure 5.1. Simulated amplitude $\left|E_{r}{ }^{*} E_{s}\right|$ and percent change $100\left(\omega_{p} / 2 \omega_{r} \beta_{x}-1\right)$ in "measured" velocity compared to the component of the velocity along the optical axis for a smooth surface.

We were intrigued that the deviations in the simulated "measured" velocity $100\left(-1+\omega_{p} / 2 \omega_{r} \beta_{x}\right)$ were so close to zero for a smooth symmetric experiment, and wanted to "see" what was happening in the summation. For each time step, and after integrating over the lens aperture but before integrating over initial positions, there is a contribution to the total peak frequency associated with each targetsurface initial position in the integrand. (This situation corresponds roughly to the Section 3.6 
"Reversing the order of integration: A geometric description of the superposition of scattered fields from one target point $\vec{r}_{i 0}$ "). For the integrand remaining after integrating over the aperture in the numerator, we define an areal-density $d \omega_{y_{i 0}, z_{i 0}} / d A$ with units of frequency per area, obtained at an initial position $y_{i o}, z_{i o}$. When approximating the integrals using a summation over finite areas of equal size (in this case $\Delta y_{i 0} \Delta z_{i 0}=\Delta y_{a} \Delta z_{a}=(1.5 \lambda)^{2}$ ), the differential areas divided out and we were left with an areal-density contribution to $\omega_{p}$ given by

$$
\begin{gathered}
d \omega_{y_{i 0}, z_{i 0}} / d A=-\operatorname{Im}\left[\frac{\iint_{A} d y_{a} d z_{a} \frac{1}{R_{P_{0} A}^{2}} \frac{\partial}{\partial t}\left[\frac{O_{i a}}{R_{i a}} E_{g}\left(\vec{r}_{i}\right) e^{i \phi_{g}\left(\vec{r}_{i},-T_{i a}\right)}\right]}{\iint d y_{i 0} d z_{i 0} \iint_{A} d y_{a} d z_{a} \frac{1}{R_{P_{0} A}^{2}}\left[\frac{O_{i a}}{R_{i a}} E_{g}\left(\vec{r}_{i}\right) e^{i \phi_{g}\left(\vec{r}_{i},-T_{i a}\right)}\right]}\right] \\
\left.\rightarrow\left(d \omega_{y_{i 0}, z_{i 0}} / d A\right) \Delta y_{i 0} \Delta z_{i 0} \cong-\operatorname{Im}\left[\frac{\sum_{y a, z a} \frac{1}{R_{P_{0} A}^{2}} \frac{\partial}{\partial t}\left[\frac{O_{i a}}{R_{i a}} E_{g}\left(\vec{r}_{i}\right) e^{i \phi_{g}\left(\vec{r}_{i},-T_{i a}\right)}\right]}{\sum_{y i, z j} \sum_{y a, z a} \frac{1}{R_{P_{0} A}^{2}}\left[\frac{O_{i a}}{R_{i a}} E_{g}\left(\vec{r}_{i}\right) e^{i \phi_{g}\left(\vec{r}_{i},-T_{i a}\right)}\right]}\right]\right]
\end{gathered}
$$

Note that $\left(d \omega_{y_{i 0}, z_{i 0}} / d A\right) \Delta y_{i 0} \Delta z_{i 0}$ sums exactly to $\omega_{p}$ (when $\omega_{p}$ is also approximated on finite area elements). To examine the relative importance of the contributions of $\left(d \omega_{y_{i 0}, z_{i 0}} / d A\right) \Delta y_{i 0} \Delta z_{i 0}$ to the total $\omega_{p}$, we plotted $\left(d \omega_{y_{i 0}, z_{i 0}} / d A\right) \Delta y_{i 0} \Delta z_{i 0} / \omega_{p}$ as a function of target-point initial positions in Fig. 5.2. Because the laser field power density falls to zero for large enough transverse distance $\left|r_{i \perp}\right|$, in the simulation the limits of integration need only span a finite subarray with center adjusted to the initial position that will end up on the beam centerline position $x_{i c}$ for the current time step. The plots are "centered." At the beam focus (Fig. 5.2 (A)), the integrand has largest contributions from those initial positions that will be on the beam centerline. 

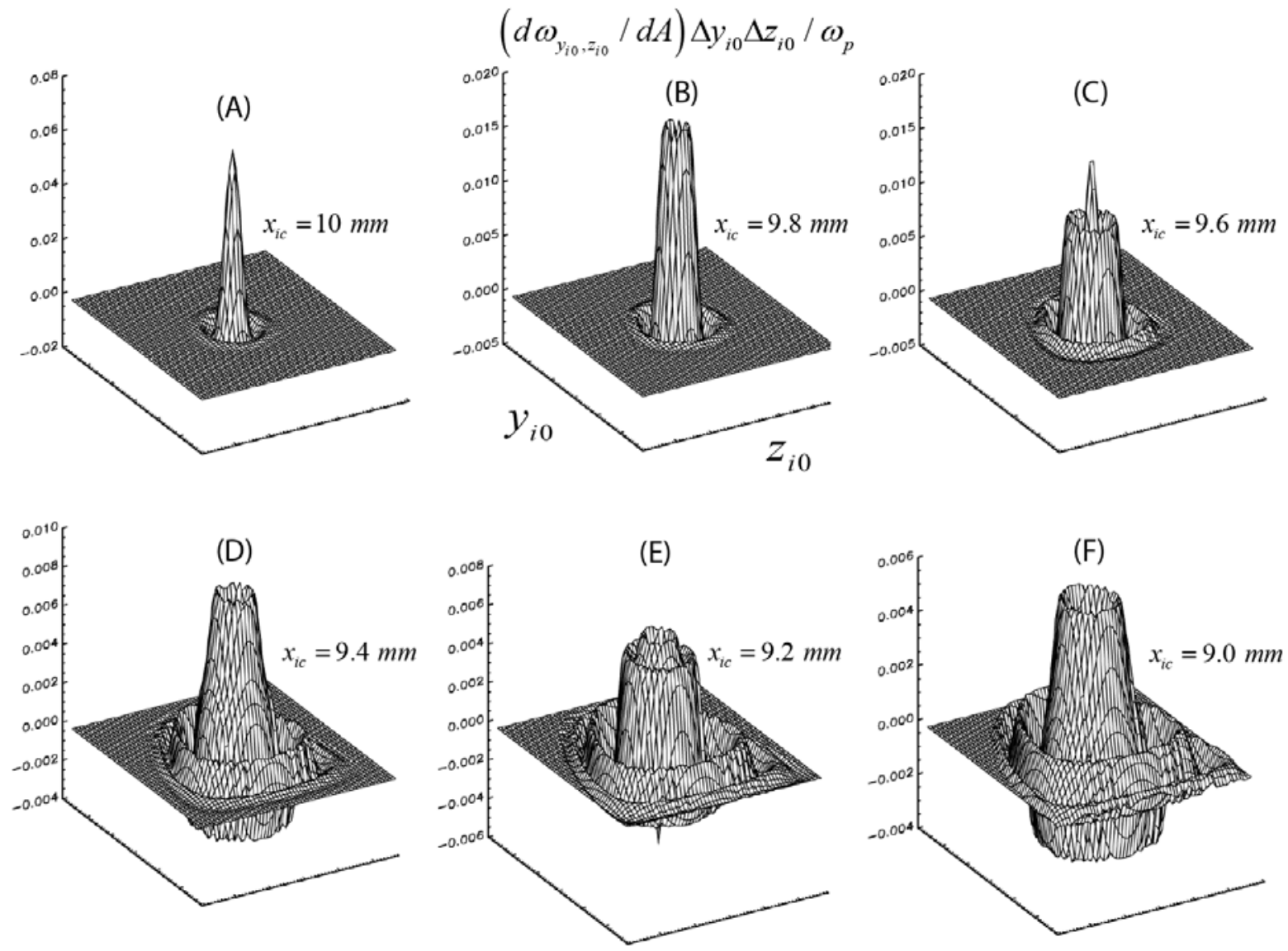

Fig. 5.2 Surface plots of the frequency contributions to the integrand $\left(d \omega_{y_{i 0}, z_{i 0}} / d A\right) \Delta y_{i 0} \Delta z_{i 0} / \omega_{p}$ associated with initial positions $y_{i 0}, z_{i 0}$ normalized to the instantaneous sideband frequency $\omega_{p}$ ( $\omega_{p}$ is the total of $\left.\left(d \omega_{y_{i 0}, z_{i 0}} / d A\right) \Delta y_{i 0} \Delta z_{i 0}\right)$. In the simulation, the target-surface had intersected the beam centerline at $x_{i c}$ values close to (A) $10 \mathrm{~mm}$, (B) $9.8 \mathrm{~mm}$, (C) $9.6 \mathrm{~mm}$, (D) $9.4 \mathrm{~mm}$, (E) $9.2 \mathrm{~mm}$, and (F) $9.0 \mathrm{~mm}$. The horizontal axes are the initial position coordinates $y_{i 0}, z_{i 0}$ that lead to $y_{i}, z_{i}$ values with range centered on the optical axis (horizontal axes are 132 micrometers wide). These figures correspond to the simulation shown in Figure 5.1.

As the target surface moves away from the focus and enters a region of curved phase fronts for the laser beam (Fig. 5.2 (b) to (f)), the contributions from the integrand $\left(d \omega_{y_{i 0}, z_{i 0}} / d A\right) \Delta y_{i 0} \Delta z_{i 0} / \omega_{p}$ begin to take on both positive and negative values. Contributions to the measured velocity oscillate with distance from the beam centerline with partial cancellations. Close examination of Fig. 5.2 (F) shows that the symmetry is not axial. Due to tilt, the $\left(d \omega_{y_{i 0}, z_{i 0}} / d A\right) \Delta y_{i 0} \Delta z_{i 0} / \omega_{p}$ surface is shaped somewhat like the Greek letter $\Delta$. A completely remarkable "coincidence" emerges: the relative velocities $\omega_{p} / 2 \omega_{r} \beta_{x}$ in Fig. 5.1 are nearly 1 to within $0.05 \%$, i.e., the measured velocity is nearly the component along the 
optical axis to within $0.05 \%$, even though the individual contributions oscillate about zero. For a smooth surface, the oscillations in the contributions to $\omega_{p}$ tend to cancel.

\subsection{Results with increasing amounts of surface roughness}

We added small values of static roughness to the target surface. We added static roughness to an initial position array. The integration sub array walks along the target surface with the center of the integration sub array at the initial position that will be on the beam centerline at simulation time t. To create static roughness for the simulation, we created a large initial position array with dimensions large enough that all initial position subarrays could be taken from the larger initial position array. We computed the target surface initial positions $x_{i 0}=x_{i 0}\left(y_{i 0}, z_{i 0}\right)$. Then we added a "roughness" surface $\delta x_{i 0}=\delta x_{i 0}\left(y_{i 0}, z_{i 0}\right)$ to the initial position plane. The roughness $\delta x_{i 0}$ is a normally distributed random variable with a standard deviation equal to the "roughness." We randomized the seed value for a random-normal distribution so that each "experiment" would see a different surface (but the surface within a simulation run would be unchanged).

When we add a small amount of static surface roughness, the $\omega_{y_{i 0}, z_{i 0}} / \omega_{p}$ plots also become rough. The oscillating patterns shown in Fig. 5.2 take on a roughened or noisy appearance. With large enough random roughness, the $\omega_{y_{i 0}, z_{i 0}} / \omega_{p}$ plots also take on a random quality swinging positive and negative rapidly with largest features in the middle of the beam.

Figures 5.3 show the amplitude and frequency results for a sequence of simulations with increasing amounts of static roughness. The values written under the panel labels are the one-standard deviation values for the roughness in micrometers ((A) 0.0, (B) 0.19, (C) 0.285, (D), (E), and (F) 0.38 micrometers).

Figure $5.3(\mathrm{~A})$ is the same as Fig. 5.1 with no roughness. When we add roughness, speckle-like amplitude modulation appears in the upper panels, similar to what is observed in a PDV experiment. In simulations with roughness, we see also larger variations in the apparent velocity. In Fig. 5.3 (B), a one standard deviation roughness of 0.19 micrometers was added (approximately one eighth of a wavelength). At 0.19 microns roughness, the velocity deviations increased a factor of 8 from $0.05 \%$ to $\pm 0.2 \%$. Fig. 5.3 (C) and (D)-(F) have larger roughness values of 0.285 and 0.38 micrometers.

The round trip phase noise from a surface with 0.38 micron roughness is about half a wavelength, where we expect largest constructive and destructive amplitude contributions to the signal amplitude. At 0.38 microns roughness, there are a few places where the amplitude is nearly zero after integrating over both the laser beam and lens aperture (almost complete signal amplitude cancellation is possible at a quarter of a wavelength roughness). At the locations where the signal amplitude dips due to surface roughness, the PDV simulation returns velocity spikes (for constant velocity of the target). The velocity spikes can swing as much as 1 or 2 percent during amplitude dips. Between the spikes, we obtain the result that the velocity is nearly the component along the optical axis to within a few tenths of a percent. 

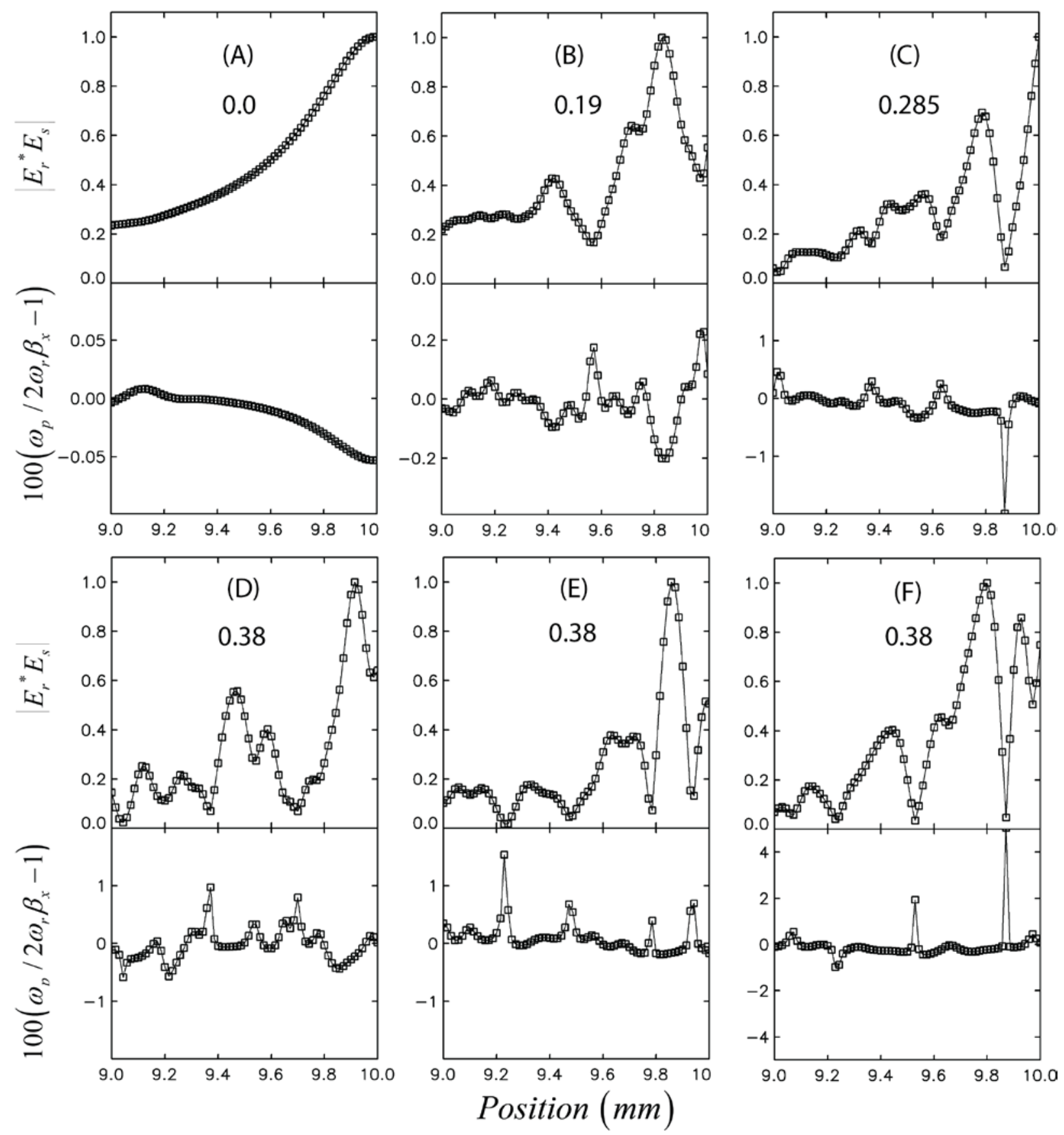

Figure 5.3. Amplitude and percent change in velocity away from the component along the optical axis for different amounts of surface roughness (values below the plot label letters are one standard deviation in the roughness in micrometers).

The last three panels of Fig. 5.3 (D), (E), (F) were all obtained with the same standard deviation of roughness ( 0.38 micrometers) but with a different initial static distribution of $\delta x_{i 0}=\delta x_{i 0}\left(y_{i 0}, z_{i 0}\right)$ values. These three panels illustrate that similar but different simulations produce similar but different results. The reader may obtain some sense of the possible behaviors from this limited set of 
simulations. (The total simulation run time for six panels in Fig. 5.3 was approximately 120 hours on a single processor-core laptop computer).

In Chapter 1 Figs. 1.3.1 and 1.3.2, we presented velocity records for an experiment with nominally identical channels. In those data, the typical random velocity noise was less than 1 percent. But in all channels there were examples at amplitude dips where the velocity spiked away from the mean value, sometimes more than 1 percent. The roughness of the aluminum coating in that experiment was on the order of 0.2 microns average roughness.

Based on the comparison of experiments and simulations, it seems plausible that the effect of roughness causes not only the expected speckle modulation of the signal amplitude, but also is connected with deviations in the apparent velocity during amplitude dips associated with destructive interference over the lens aperture. These can be understood by examining Fig. 5.2 where we realize that peak frequency contributions have an oscillatory nature over the target position after integrating over the lens. Adding roughness is sometimes necessary to decrease the sensitivity to tilt in PDV. A recommendation based on these simulations is to avoid increasing the roughness to as large as one quarter of the wavelength. Another useful feature of these results comes with an understanding of a possible origin of velocity spikes. When averaging results over nominally identical channels, not only do we want to ignore results where the amplitude is very low, it is recommended that one view regions where the amplitude is racing to a low value with suspicion.

We should comment that there will be increased uncertainty in the velocity for decreased signal to noise ratio. But the random effect of random noise is not what is happening in these simulations since we carefully avoided the random noise issues in the signal by taking our simulation into the large field classical limit with no random noise contribution (sections 2.2 and 3.1). The velocity spikes in this work are from unusual summations over complex fields on a rough surface. 


\section{BIBLIOGRAPHY}

D. H. Dolan, "What Does 'Velocity' Interferometry Really Measure?," CP1195 Shock Compression of Condensed Matter, Eds: M. L.Elert, W. T. Buttler, M. D. Furnish, W. W. Anderson, and W. G. Proud, an American Institute of Physics Conference Proceeding 1195, 589 (2009).

M. E. Briggs, L. M. Hull, and M. A. Shinas, "Fundamental Experiments in Velocimetry," CP1195 Shock Compression of Condensed Matter, Eds: M. L.Elert, W. T. Buttler, M. D. Furnish, W. W. Anderson, and W. G. Proud, an American Institute of Physics Conference Proceeding 1195, 577 (2009).

D. H. Dolan, "Accuracy and precision in photonic Doppler velocimetry," Review of Scientific Instruments, Vol 81, 053905 (2010).

E. A. Moro and M. Briggs, "Note: Simultaneous Measurement of Transverse Speed and Axial Velocity from a Single Optical Beam," Review of Scientific Instruments, Vol 84, 016110 (2013).

O. T. Strand, D. R. Goosman, C. Martinez, T. L. Whitworth, and W. W. Kuhlow, "Compact system for highspeed velocimetry using heterodyne techniques," Review of Scientific Instruments, Vol 77, 083108 (2006).

O. T. Strand Retrospective presentation at the PDV workshop at the University of Nevada, Las Vegas (June 2014).

R. H. Kingston, "Detection of Optical and Infrared Radiation," Springer Series in Optical Sciences, Vo. 10, Ed. David L. MacAdam, (Springer-Verlag, New York, 1978).

A. Yariv, "Optical Electronics," (Holt Rinehart Winston, New York, 1985).

J. D. Jackson, “Classical Electrodynamics," Second Edition, (Wiley, New York 1975).

J. Goodman, “Introduction to Fourier Optics," (McGraw-Hill, New York, 1968).

M. Born and E. Wolf, "Principles of Optics," $6^{\text {th }}$ edition (Pergamon, New York, 1980).

P. Lorrain and D. R. Corson, "Electromagnetic Fields and Waves," Second Edition, (Freeman, San Francisco 1970).

G. R. Fowles, "Introduction to Modern Optics," Second Edition (Holt Rinehart Winston, New York, 1975). 